\title{
Procedure to Generate the MPACT Multigroup Library
}

\author{
Revision 0 \\ December 17, 2015
}

Kang Seog Kim


Approved for public release.

Distribution is unlimited.

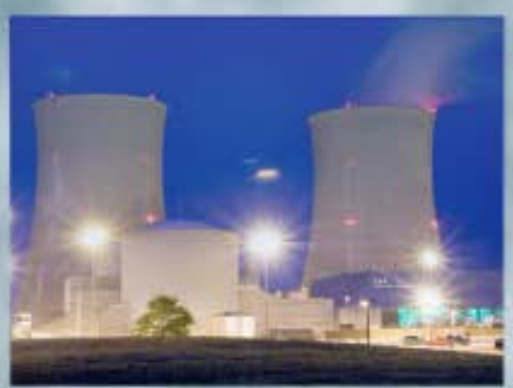




\section{REVISION LOG}

\begin{tabular}{|c|c|c|l|}
\hline $\begin{array}{c}\text { Revisio } \\
\mathbf{n}\end{array}$ & Date & Affected Pages & \multicolumn{1}{c|}{ Revision Description } \\
\hline 0 & $12 / 17 / 2015$ & All & Initial version \\
\hline & & & \\
\hline & & & \\
\hline & & & \\
\hline
\end{tabular}

\section{Document pages that are:}

Export Controlled None

IP/Proprietary/NDA Controlled None

Sensitive Controlled None

Unlimited $\underline{\text { All }}$

\section{Requested Distribution:}

To: N/A

Copy: N/A

\section{Reviewed by:}

Date:

Reviewer: Kevin Clarno 


\section{DOCUMENT AVAILABILITY}

Reports produced after January 1, 1996, are generally available free via US Department of Energy (DOE) SciTech Connect.

Website http://www.osti.gov/scitech/

Reports produced before January 1, 1996, may be purchased by members of the public from the following source:

National Technical Information Service

5285 Port Royal Road

Springfield, VA 22161

Telephone 703-605-6000 (1-800-553-6847)

TDD 703-487-4639

Fax 703-605-6900

E-mailinfo@ntis.gov

Website http://www.ntis.gov/help/ordermethods.aspx

Reports are available to DOE employees, DOE contractors, Energy Technology Data Exchange representatives, and International Nuclear Information System representatives from the following source:

Office of Scientific and Technical Information

PO Box 62

Oak Ridge, TN 37831

Telephone 865-576-8401

Fax 865-576-5728

E-mail reports@osti.gov

Website http://www.osti.gov/contact.html

This report was prepared as an account of work sponsored by an agency of the United States Government. Neither the United States Government nor any agency thereof, nor any of their employees, makes any warranty, express or implied, or assumes any legal liability or responsibility for the accuracy, completeness, or usefulness of any information, apparatus, product, or process disclosed, or represents that its use would not infringe privately owned rights. Reference herein to any specific commercial product, process, or service by trade name, trademark, manufacturer, or otherwise, does not necessarily constitute or imply its endorsement, recommendation, or favoring by the United States Government or any agency thereof. The views and opinions of authors expressed herein do not necessarily state or reflect those of the United States Government or any agency thereof. 


\section{EXECUTIVE SUMMARY}

The CASL neutronics simulator MPACT is under development for the neutronics and T-H coupled simulation for the light water reactor. The objective of this document is focused on reviewing the current procedure to generate the MPACT multigroup library. Detailed methodologies and procedures are included in this document for further discussion to improve the MPACT multigroup library. 


\section{TABLE OF CONTENTS}

REVISION LOG

EXECUTIVE SUMMARY

TABLE OF CONTENTS

ACRONYMS

1. INTRODUCTION

2. MULTIGROUP CROSS SECTION PROCESSING BY AMPX

2.1 Conventional AMPX procedure

2.2 Practical Weighting function

3. GENERATION OF IMPROVED RESONANCE DATA

3.1 Slowing down equation

3.2 Intermediate resonance approximation

3.3 Intermediate resonance parameters

3.4 Resonance self-shielded data by homogeneous models

3.5 Resonance self-shielded data by heterogeneous models

iii

\section{$\mathrm{V}$}

vi

1

2

2

3

5

3.6 Resonance self-shielded data by Monte Carlo code

3.7 Unresolved resonance self-shielded data

3.8 Self-shielded data for within-group elastic scattering

4. RESONANCE SELF-SHIELDING METHODS

4.1 Subgroup method

4.2 Subgroup data generation

4.3 Subgroup method for non-uniform temperature distribution 20

$\begin{array}{ll}\text { 4.4 Embedded self-shielding method } & 21\end{array}$

5. GENERATION OF MISCELLANEOUS DATA 22

$\begin{array}{ll}5.1 \text { Transport cross sections for }{ }^{1} \mathrm{H} & 22\end{array}$

$\begin{array}{ll}5.2 \text { Transient data } & 24\end{array}$

5.3 Effective recoverable energy per fission $\quad 25$

5.4 Effective background cross sections $\quad 26$

6. GENERATION OF THE MPACT MG LIBRARY 28

6.1 Generation of the initial AMPX MG library $\quad 28$

6.2 Intermediate resonance parameters and Homogeneous F-factors 29

6.3 Heterogeneous F-factors $\quad 30$

6.4 Generation of subgroup data 31

6.5 Generation of the MPACT MG library 33

7. CHALLENGING ISSUE

7.1 Drawbacks of the MPACT MG library 37

$\begin{array}{ll}7.2 \text { Resonance interference } & 37\end{array}$

7.3 Resonance self-shielding effect on scattering matrix 37

7.4 Intra pin self-shielding effect $\quad 38$

ACKNOWLEDGEMENT $\quad 39$

REFERENCE $\quad 40$

Appendix A.1 Sample input of LAMBDA and IRFFACTOR-hom for ${ }^{235} U$

Appendix B.1 Sample input of IRFFACTOR-het for ${ }^{238} U$

Appendix C.1 Data information in the MAPCT 47-group library 48

Appendix C.2 DECLIB input to generate the ENDF/B-7.1 V4.0 MPACT 47-group library 52 


\section{FIGURES}

Figure 2.2.1 Pointwise neutron spectra 4

Figure 3.1.1 Flowchart of modules used to generate problem-dependent MG data $\quad 6$

Figure 3.1.2 Definition of UMR, PW, and LMR energy ranges $\quad 7$

Figure 3.6.1 Procedure to generate resonance data with epithermal upscattering 13

Figure 4.1.1 Resonances and subgroups 16

Figure 5.1.1 47-group transport correction factors for ${ }^{1} \mathrm{H} \quad 23$

Figure 5.2.1 47-group weighting function $\quad 24$

Figure 5.2.2 Transient data from ENDF/B-7.0 25

Figure 6.1.1 Sample template to generate the AMPX input files 28

Figure 6.1.2 Flow chart of the AMPX procedure $\quad 29$

Figure 6.2.1 Sample template to generate the IR parameters and homogeneous F-factors 30

Figure 6.2.1 Flow chart of the resonance data generation procedure 30

Figure 6.3.1 Sample input to generate the heterogeneous F-factors 31

Figure 6.4.1 Sample of the initial subgroup level 32

Figure 6.4.2 Sample of the SUBGR input 33

Figure 6.5.1 DECLIB calculation flow 34

Figure 7.3.1 Counter influence between fuel and cladding 38

\section{TABLES}

Table 3.5.1 Example of variations for ${ }^{238} \mathrm{U} \quad 10$

Table 6.4.1 List of nuclides including subgroup data 32

Table 6.5.1 MT numbers to be merged $\quad 36$ 


\section{ACRONYMS}

0D Zero-Dimensional (Homogeneous)

1D One-Dimensional

2D Two-Dimensional

CASL Consortium for Advanced Simulation of Light Water Reactors

CE Continuous Energy (as in cross sections)

ESSM Embedded Self-Shielding Method

IR Intermediate Resonance

MG Multi-group (as in cross sections)

MOC Method of Characteristics

NR Marrow Resonance

ORNL Oak Ridge National Laboratory

PW Pointwise

PWR Pressurized Water Reactor

RI Resonance Integral

URR Unresolved resonance

WR Wide Resonance 



\section{INTRODUCTION}

The main neutronic simulator in the Consortium for Advanced Simulation of Light Water Reactors (CASL) is the MPACT code which is being developed collaboratively by Oak Ridge National Laboratory and University of Michigan. The key characteristics of the MPACT code includes the subgroup method [Hel03] and the embedded self-shielding method (ESSM) [Wil12] for resonance self-shielding calculation, and a whole core solver with a 2D/1D synthesis method on the frame of 3D coarse mesh finite difference (CMFD) method for which axial and radial correction factors are obtained from $1 \mathrm{D}$ nodal expansion method (NEM) or Simplified $\mathrm{P}_{\mathrm{N}}$ and 2D method of characteristics (MOC), respectively. The multigroup (MG) cross section library for MPACT has been generated with significant extensions of the AMPX [Wia14] and SCALE [Sca09] code packages developed at Oak Ridge National Laboratory for which the AMPX code package has been significantly improved especially for the Bondarenko approach of resonance self-shielding calculation such as the subgroup method and ESSM used in MPACT.

The objective of this document is focused on reviewing the current procedure to generate the MPACT MG library. Detailed methodologies and procedures for the generation of the MPACT MG library are included in this document for further discussion to improve the library. This document includes the followings.

- $\quad$ MG cross section processing by AMPX (Chapter 2)

- Improvement of resonance data (Chapter 3)

- Resonance self-shielding methods (Chapter 4)

- Generation of miscellaneous data (Chapter 5)

- Generation of the MPACT MG library (Chapter 6)

- Challenging technical issues (Chapter 7) 


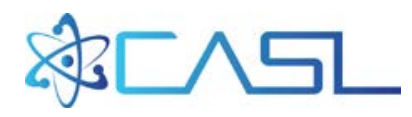

\section{MULTIGROUP CROSS SECTION PROCESSING BY AMPX}

\section{$\underline{2.1 \text { Conventional AMPX procedure }}$}

AMPX-6 [Wia14] is a modular system of FORTRAN computer programs to generate the MG and continuous energy (CE) cross section libraries for modern deterministic and Monte Carlo transport codes by processing the ENDF/B libraries. Since the CASL neutronics simulator MPACT is a deterministic transport code, only the AMPX MG library generation procedure is discussed.

The AMPX MG library includes various neutron reactions of the Bondarenko F-factors as a function of background cross section for all energy groups including resolved and unresolved resonances. The resolved resonance F-factors have been generated by the narrow resonance (NR) approximation and the unresolved ones by the J-function method [Lee11] based on the NR approximation. And MG 1D and 2D cross section data have been obtained by using a weighting function of Maxwellian spectrum $+1 / E+$ fission spectrum. At low energies, the weighting function is a Maxwellian spectrum which has a flux shape that assumes the neutron scatters into a region with a free gas scatterer that has no absorption. The Maxwellian flux spectrum has the following formula.

$$
\phi(E)=M(E)=E e^{-\frac{E}{k T}},
$$

where $E, k, T$ denote neutron energy, Boltzmann constant and temperature of the material in Kelvin, respectively. In the slowing down range, $0.125 \mathrm{eV}<E<E_{c u t}$, the weighting spectrum is assumed to be $\phi(E)=1 / E$. The cutoff energy $E_{c u t}$ for the slowing down range must be selected and is typically $55 \mathrm{keV}$ by default in the AMPX modules. In the region, $E_{\text {cut }}<E<10^{7} \mathrm{eV}$, where fission neutrons are born, the following fission spectrum is used.

$$
\phi(E)=\chi(E)=E^{0.5} e^{-\frac{E}{\theta}}
$$

where $\theta$ is temperature of the fission spectrum (e.g., $1.2 \times 10^{6} \mathrm{eV}$ ). For energies above $10^{7} \mathrm{eV}$, the particles are considered to be in another slowing down region; hence, the spectrum is assumed to have a $1 / E$ shape.

MG self-shielded cross sections of reaction type $i$ (Bondarenko F-factors) as a function of background cross sections $\left(\sigma_{0}\right)$ and material temperature (T)are calculated by using the following equation based on narrow resonance approximation.

$$
\sigma_{i, g}\left(T, \sigma_{0}\right)=\frac{\int_{g} \frac{\sigma_{i}(T, E) \sigma_{0} \phi(E)}{\sigma_{t}(T, E)+\sigma_{0}} d E}{\int_{g} \frac{\sigma_{0} \phi(E)}{\sigma_{t}(T, E)+\sigma_{0}} d E},
$$


where $\mathrm{T}$ denotes temperature and $\sigma_{t}$ is total cross section. The MG scattering matrix can be obtained by

$$
\sigma_{s, l, g g^{\prime}}=\frac{1}{\int_{g} \phi(E) d E} \int_{g} y(E) \sigma_{s}(E) \phi(E) d E \int_{g^{\prime}} f_{l}\left(E, E^{\prime}\right) d E^{\prime},
$$

where $y(E)$ is a multiplicity which is unity for scattering and $f_{l}\left(E, E^{\prime}\right)$ a normalized double differential distribution. It should be noted that the 2-D scattering data (not for 2-D thermal scattering) are temperature independent in the AMPX MG library.

\subsection{Practical weighting function}

In the standard SCALE sequences (e.g. TRITON), self-shielded MG 1D and 2D data for resolved resonance and thermal energy groups are determined by performing the problemdependent CENTRM slowing down transport calculation for each pin cell type with eq. (2.2.1), and thus the resolved resonance data in the AMPX MG library are not used.

$$
\hat{\Omega} \cdot \nabla \psi+\Sigma_{t}(\vec{r}, u) \psi(\vec{r}, u, \hat{\Omega})=\int_{4 \pi} d \Omega^{\prime} \int_{0}^{\infty} d u \Sigma_{s}\left(\vec{r}, u^{\prime} \rightarrow u, \hat{\Omega}^{\prime} \cdot \hat{\Omega}\right) \psi\left(\vec{r}, u^{\prime}, \hat{\Omega}^{\prime}\right)+q(\vec{r}, u, \hat{\Omega}),
$$

where

$$
\begin{array}{ll}
\hat{\Omega} & : \text { neutron direction } \\
\vec{r} & : \text { space coordinate } \\
u & : \text { neutron lethargy } \\
\psi & : \text { angular flux } \\
\Sigma_{t} & : \text { macroscopic total cross section } \\
\Sigma_{s} & : \text { double differential scattering cross section } \\
q & : \text { external source. }
\end{array}
$$

However, since Bondarenko approach for resonance self-shielding calculation does not include any problem-dependent pointwise slowing down calculation, weighting function would make a significant impact on the accuracy mostly through 2-D scattering matrix. The weighting function of Maxwellian spectrum $+1 / E+$ fission spectrum is far from a realistic weighting function. Practical weighting functions for various temperatures can be obtained by performing the CENTRM MG/PW calculations for a typical PWR fuel pin. Figure 2.2.1 provides a sample of pointwise neutron spectra obtained by CENTRM. This would be performed using the XSProc sequence with "parm=centrm". The weight functions need to be generated to include various temperatures.

There are several pending issues associated with the pointwise weighting function. Since resonance self-shielded cross sections are independently estimated, 1D cross sections are independent upon the pointwise weighting function. However, in reality since the current MPACT procedure includes resonance data only for the specified energy groups, 1D cross 


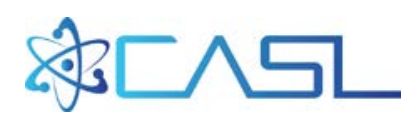

sections of non-resonance groups should be dependent on the weighting functions. MG scattering matrices are obtained by simple flux weighting and renormalization is applied only to total scattering, within-group and out-scattering components. Therefore, this procedure may introduce wrong neutron fluxes resulting in some errors in reaction rates. Pointwise neutron spectra are very changeable according to ${ }^{235} \mathrm{U}$ enrichment, moderator-to-fuel ratio, burnup and void fraction. Therefore, selection of weighting function would be another challenging issue.

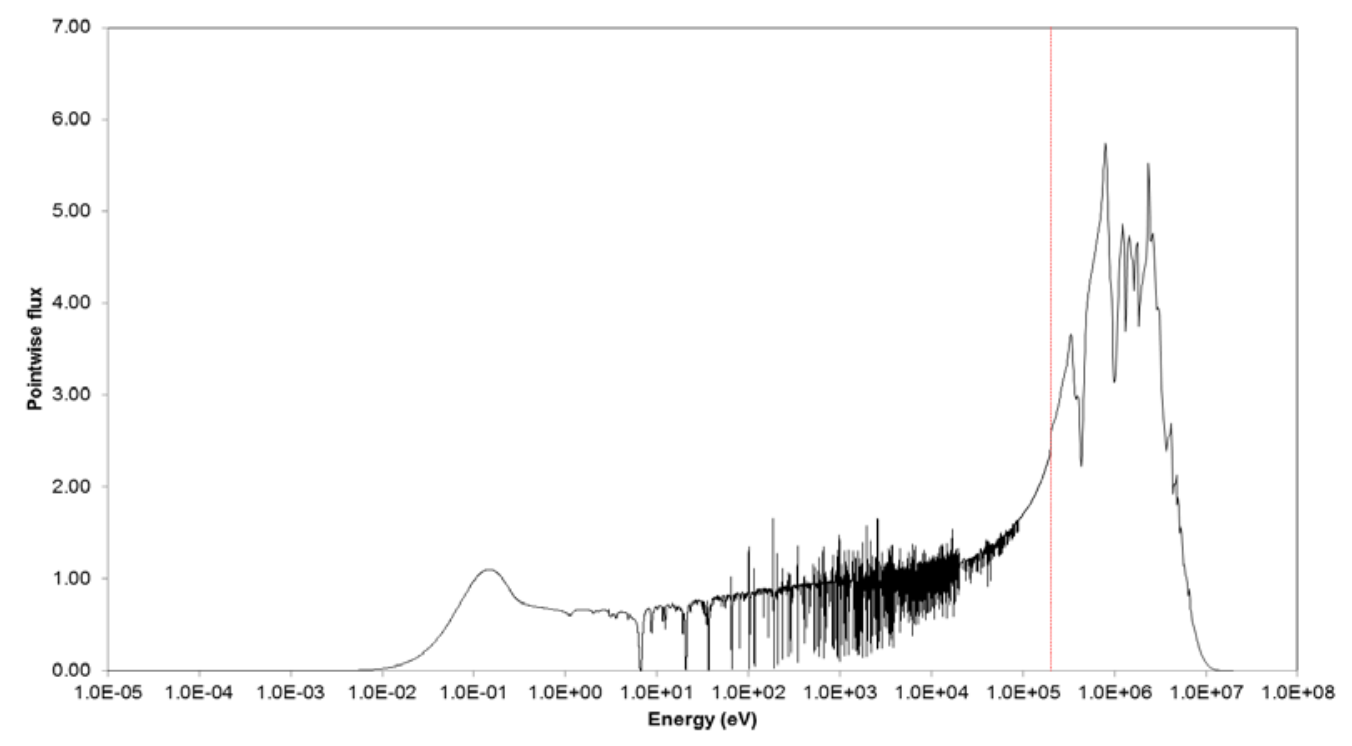

Figure 2.2.1 Pointwise neutron spectra 


\section{GENERATION OF IMPROVED RESONANCE DATA}

Although the AMPX MG library includes MG resonance self-shielded cross sections (Bondarenko F-factors) as a function of background cross sections generated by eq. (2.1.3), they cannot guarantee accurate result due to significant approximations such as coarse energy group, weighting functions and resonance interferences. Therefore, the MG resonance self-shielded data need to be improved in accordance with resonance self-shielding method. This chapter describes several methods to improve the resonance self-shielded cross section data.

\subsection{Slowing down equation}

The time-independent Boltzmann equation is as follows:

$$
\hat{\Omega} \cdot \nabla \psi+\Sigma_{t}(\vec{r}, u) \psi(\vec{r}, u, \hat{\Omega})=\int_{4 \pi} d \Omega^{\prime} \int_{0}^{\infty} d u^{\prime} \Sigma_{s}\left(\vec{r}, u^{\prime} \rightarrow u, \hat{\Omega}^{\prime} \cdot \hat{\Omega}\right) \psi\left(\vec{r}, u^{\prime}, \hat{\Omega}^{\prime}\right)+q(\vec{r}, u, \hat{\Omega}),
$$

where

$$
\begin{array}{ll}
\hat{\Omega} & : \text { neutron direction } \\
\vec{r} & : \text { space coordinate } \\
u & : \text { neutron lethargy } \\
\psi & : \text { angular flux } \\
\Sigma_{t} & : \text { macroscopic total cross section } \\
\Sigma_{s} & : \text { double differential scattering cross section } \\
q & : \text { external source. }
\end{array}
$$

The continuous scalar flux can be obtained by solving eq. (3.1.1) using the point-wise cross sections. The effective group self-shielded cross section of the resonance isotope can be obtained using the equation (3.1.2):

$$
\sigma_{x, g}=\frac{\int_{\Delta u_{g}} \sigma_{x}(u) \phi(u) d u}{\int_{\Delta u_{g}} \phi(u) d u} .
$$

In the SCALE code package CENTRM and PMC perform the above calculations to obtain the group self-shielded cross sections for various geometrical models including infinite medium, slab, 1D cylinder and sphere and 2D square pin.

A flowchart of the SCALE modules executed for self-shielding is shown in Figure 3.1.1. First, the fast-executing Bondarenko method is applied to all energy groups for the total, elastic, capture, and fission cross sections of all materials by using BONAMI. In this method MG data at problem-specific conditions are interpolated from the tabulated self-shielding factors in the MG library. While the analytical flux expression used to process Bondarenko shielding factors is reasonable for higher energy groups, it tends to break down at lower energies. SCALE 6 
provides other approaches to generate problem-dependent MG data directly from PW data within the energy range in which the Bondarenko method is not desirable - typically the resolved resonance and thermal ranges for criticality safety and reactor physics applications. As shown in Figure 3.1.2, the energy range of interest is divided into the upper multigroup (UMR), PW, and lower multigroup (LMR) ranges, as defined by the input energy boundaries $E_{\max }$ and $E_{\min }$, which have default values of $20 \mathrm{keV}$ and $10^{-3} \mathrm{eV}$, respectively, in SCALE 6. Generally it is recommended that the PW energy range should encompass the resolved resonance ranges of all nuclides that impact the spectral fine-structure; however MG data in the UMR and LMR are always self-shielded with the Bondarenko method. Thus it is not necessary to perform a PW calculation within the energy range where the Bondarenko approximation is adequate. This is done by the CENTRM code, which computes neutron spectra on a fine energy mesh within the PW energy range using various approximations to the Boltzmann equation. In the energy region outside the specified PW range, CENTRM performs an MG calculation so that a full energy range solution is obtained.

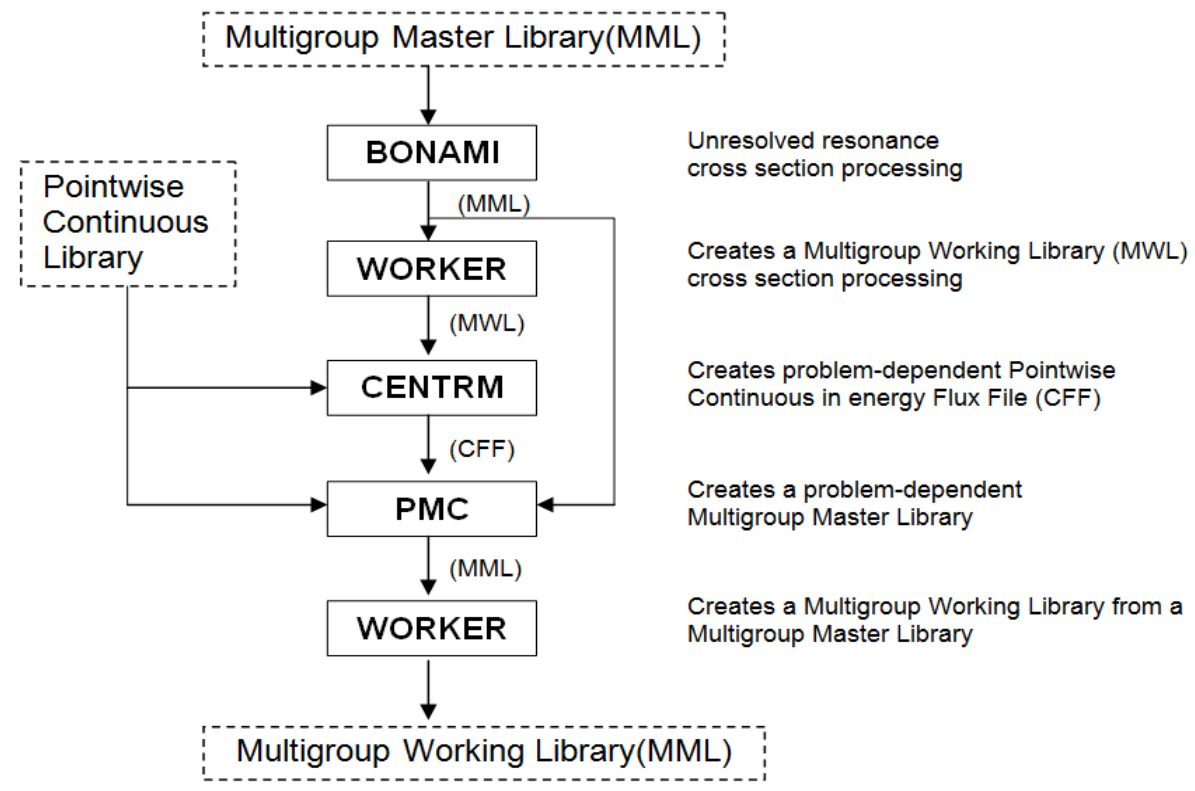

Figure 3.1.1 Flowchart of modules used to generate problem-dependent MG data

The PMC module computes problem-specific MG data for groups within the PW energy range of the CENTRM calculation and replaces the more approximate values from BONAMI. PMC uses the CENTRM spectra for each spatial region as a problem-dependent weight function for averaging energy-dependent data in the PW libraries into MG data. The resulting problemspecific cross-section library is passed to higher-dimensional MG transport calculations performed with other SCALE modules.

Exact same procedure can be utilized in improving MG resonance data in the AMPX MG library. The only difference is to additionally introduce the following assumptions to exclude resonance interference effect according to a user option. 
a. Only one resonant nuclide is assumed in the mixture with a constant potential scattering cross section $\left(\sigma_{p}\right)$, a resonance scattering cross section $\left(\sigma_{r s}(u)\right)$ and a resonance absorption cross section $\left(\sigma_{r a}(u)\right)$.

b. The non-resonant nuclides are considered to have negligible absorption and constant potential scattering cross sections.

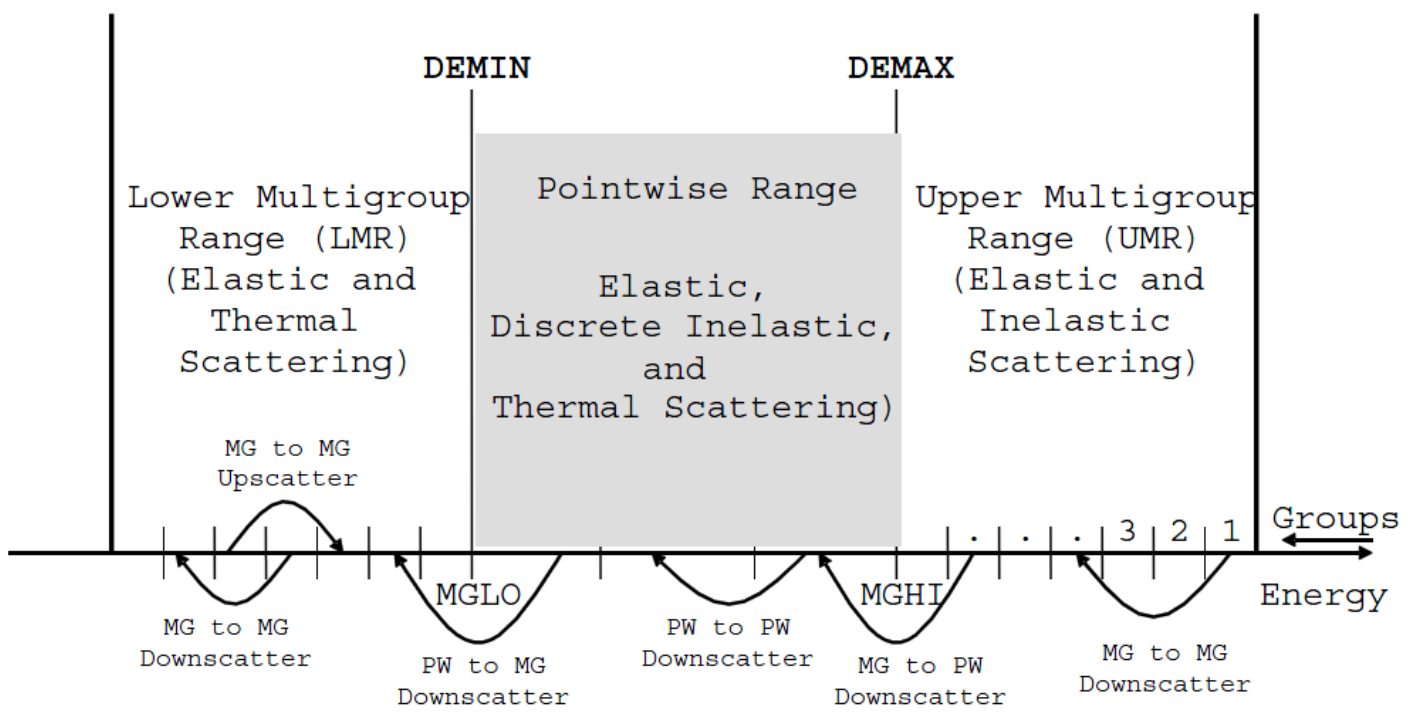

Figure 3.1.2 Definition of UMR, PW, and LMR energy ranges

Exact same procedure by using CENTRM can be utilized in improving resonance data. Several methods are available to improve resonance data which include homogeneous or heterogeneous models, and multiple absorber model to pre-consider resonance interferences or single absorber model to exclude resonance interference. A new procedure using I-CRAWDIUS has been developed to provide potential cross sections or pointwise cross sections to CENTRM for the background nuclides to exclude or include resonance interference between resonance nuclides according to user option.

\section{$\underline{3.2 \text { Intermediate resonance (IR) approximation }}$}

To cast the scattering source term in eq. (3.1.6) into a more tractable form, the intermediate resonance (IR) approximation is introduced.

In this approximation, the fraction $\lambda_{i}$ of the scattering for each isotope $i$ is assumed to be so effective that the maximum lethargy gain per collision is significantly greater than the practical resonance width (i.e., the narrow resonance scattering)[Hel03, Sta83]. A resonance affects only a small interval of the integration range from $u-\Delta_{i}$ to $u$, and its contribution to the scattering source $Q(u)$ is negligible. Since outside the resonances $\Sigma_{s i}(u)=\Sigma_{p i}$ and $\phi(u)=$ constant, the contribution 


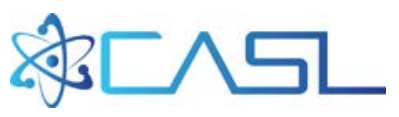

of isotope $i$ to $Q(u)$ is $\lambda_{i} \Sigma_{p i}$. This is the unperturbed slowing down source. Conversely, the remaining fraction $\left(1-\lambda_{i}\right)$ is assumed to be so ineffective that neutrons gain a negligible amount of lethargy compared with $\Delta u_{g}$ (i.e., the wide resonance scattering). The resonances are so wide that the integrand can be replaced by its average value, which leads to the $\left(1-\lambda_{i}\right) \Sigma_{s i}(u) \phi(u)$ contribution to the source $Q(u)$. Thus, this fraction of scattering does not provide source neutrons from outside the resonance widths but it should be considered as due to self-scattering which neither adds nor removes neutrons. With these approximations, eq. (3.1.6) can be rewritten for the coarse energy groups:

$$
\hat{\Omega} \cdot \nabla \psi_{g, k}+\sum_{i} \sum_{i, g, t}^{k} \psi_{g, k}(\hat{\Omega})=\sum_{i} \lambda_{i, g} \sum_{i, p}^{k}+\sum_{i}\left(1-\lambda_{i, g}\right) \sum_{i, g, s}^{k} \phi_{g, k} \cdot
$$

Eq. (3.2.1) can be rewritten by assuming isotropic angular flux as

$$
\hat{\Omega} \cdot \nabla \psi_{g, k}+\sum_{i}\left(\Sigma_{i, g, a}^{k}+\lambda_{i, g} \Sigma_{i, p}^{k}\right) \psi_{g, k}(\hat{\Omega})=\sum_{i} \lambda_{i, g} \Sigma_{i, p}^{k}
$$

For the homogeneous mixture, the self-shielded flux can be obtained from eq. (3.2.2) as

$$
\phi_{g}=\frac{\sum_{i} \lambda_{i, g} \Sigma_{i, p}}{\sum_{i=\text { resonance }} \Sigma_{i, g, a}+\sum_{i} \lambda_{i, g} \Sigma_{i, g, s}}=\frac{\lambda_{g} \Sigma_{p}}{\Sigma_{g, a}+\lambda_{g} \Sigma_{g, s}} .
$$

\section{$\underline{3.3 \text { Intermediate resonance parameters }}$}

Let us assume there is only one resonance isotope having an atomic number density $N_{R}$. In a homogeneous system, the background cross section of this mixture is defined by

$$
\sigma_{b}=\frac{\sum_{i=a l l} N_{i} \lambda_{i} \sigma_{i, p}}{N_{R}} .
$$

Eq. (3.2.1) can be rewritten by using eq. (3.3.1) as

$$
\phi_{g}=\frac{\sigma_{g, b}}{\sigma_{g, a}+\sigma_{g, b}+\lambda_{g} \sigma_{g, r s}} .
$$

Since the atomic mass of hydrogen $\left({ }^{1} H\right)$ is very close to unity, lethargy gain of any neutron colliding with hydrogen is very big and the neutron can scatter beyond a resonance without any collision. This is essentially the same as the narrow resonance approximation. Therefore, for hydrogen, the intermediate resonance parameter is defined as unity. For other isotopes, the intermediate resonance parameter is obtained by comparing results of various ${ }^{238} \mathrm{U} /{ }^{1} \mathrm{H}$ mixtures 
where the hydrogen is partly replaced by the other isotopes. This is often referred to as a hydrogen-equivalence parameter.

For homogeneous mixtures including ${ }^{238} U$ and ${ }^{1} \mathrm{H}$, eq. (3.2.1) can be written as follows:

$$
\left(N^{238} \sigma_{g, a}^{238}+N^{238} \sigma_{g, r s}^{238}+N^{238} \lambda_{g}^{238} \sigma_{p}^{238}+N^{1} \lambda_{g}^{1} \sigma_{p}^{1}\right) \phi_{g}=N^{238} \lambda_{g}^{238} \sigma_{p}^{238}+N^{1} \lambda_{g}^{1} \sigma_{p}^{1}
$$

In order to obtain the hydrogen-equivalence parameter for a nuclide, a part of hydrogen $\left(N^{1}\right)$ is replaced with a certain nuclide $\left(N^{X}\right)$. The amount of the hydrogen atomic number density replaced by another isotopic atomic number density can be adjusted to obtain the same $\sigma_{g, a}^{238}$ from the slowing down calculation by using eq. (3.1.6). The balance equation will be

$$
\begin{aligned}
&\left(N^{238} \sigma_{g, a}^{238}+N^{238} \sigma_{g, r s}^{238}+\right. N^{238} \lambda_{g}^{238} \sigma_{p}^{238}+( \\
&\left.\left.N^{1}-N^{x}\right) \lambda_{g}^{1} \sigma_{p}^{1}+N^{x} \lambda_{g}^{x} \sigma_{p}^{x}\right) \phi_{g}= \\
& N^{238} \lambda_{g}^{238} \sigma_{p}^{238}+\left(N^{1}-N^{x}\right) \lambda_{g}^{1} \sigma_{p}^{1}+N^{x} \lambda_{g}^{x} \sigma_{p}^{x}
\end{aligned}
$$

By comparing these two equations, the unknown intermediate resonance parameter $\left(\lambda^{x} g\right)$ can be obtained as follows:

$$
\lambda_{g}^{x}=\frac{\Sigma_{g, b}-N^{238} \lambda_{g}^{238} \sigma_{p}^{238}-\left(N^{1}-N^{x}\right) \lambda_{g}^{1} \sigma_{p}^{1}}{N^{x} \sigma_{p}^{x}},
$$

where

$$
\Sigma_{g, b}=N^{238} \lambda_{g}^{238} \sigma_{p}^{238}+N^{1} \lambda_{g}^{1} \sigma_{p}^{1}
$$

In the actual application, it is too time consuming to determine the fraction of the hydrogen atomic number density that needs to be replaced with a target nuclide. Therefore, the following procedure can be used instead using data derived from ${ }^{238} \mathrm{U}$ data.

(a) Compute a $\sigma_{g, a}^{238}$ table as a function of $\sigma_{g, b}^{238}$ by changing the particle number density of hydrogen for a homogeneous mixture at the fixed ${ }^{238} U\left(N^{238}\right)$ particle number density.

(b) Compute the slowing down calculation for a mixture of ${ }^{238} U\left(N^{238}\right),{ }^{1} H\left(N^{1}\right)$ and a target nuclide $x\left(N^{x}\right)$, and obtain a new $\sigma_{g, a}^{238}$.

(c) Read the corresponding $\sigma_{g, b}^{238}$ from the prepared $\sigma_{g, a}^{238}$ table.

(d) Calculate the hydrogen equivalent parameter using the following equation.

$$
\lambda_{g}^{x}=\frac{\sigma_{g, b}^{238} N^{238}-N^{238} \lambda_{g}^{238} \sigma_{p}^{238}-N^{1} \lambda_{g}^{1} \sigma_{p}^{1}}{N^{x} \sigma_{p}^{x}} .
$$

Intermediate resonance parameters can be also calculated for the other resonance nuclide such as ${ }^{235} \mathrm{U},{ }^{239} \mathrm{Pu}$ and others. 


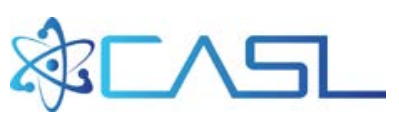

\subsection{Resonance self-shielded data by homogeneous models}

The energy dependence of the cross sections in the library has been discretized by dividing the energy range of interest, $10^{-5} \mathrm{eV} \sim 10 \mathrm{MeV}$, into a number of broad groups. These cross sections have been obtained by flux-averaging point XS - some-times more than $10^{5}$ points with typical reactor spectra. However, it is unfortunate that this procedure is impractical for the resonance isotopes in the range from $100 \mathrm{KeV} 1 \mathrm{eV}[4,5]$. In this range, the cross sections exhibit many resonances and hence so that thousands of energy groups would be required for a satisfactory discretization. In general, the number of resonance groups is limited to 5 30 for very coarse group structures including 50 60 energy groups, though SCALE generally uses well over 150 . The objective of the resonance treatment is to evaluate the effective cross section for the resonance isotopes in the all resonance energy groups:

$$
\sigma_{x, g}=\frac{\int_{\Delta u_{g}} \sigma_{x}(u) \phi(u) d u}{\int_{\Delta u_{g}} \phi(u) d u} .
$$

In eq. (3.4.1), the lethargy $\left(u=\ln \left(E_{0} / E\right), E_{0}=10 \mathrm{MeV}\right)$ is used instead of the neutron energy and $x$ represents a reaction type. However, the library data available for this purpose are tables of group-dependent resonance integrals (RI) (i.e., the numerator of eq. (3.4.1)) versus temperatures and background cross sections. In CENTRM, the flux in eq. (3.4.1) is calculated by solving the neutron slowing down equation for homogeneous or heterogeneous system.

To describe the slowing down equation in homogeneous infinite system containing a mixture of isotopes, indexed $i$, of which one is a resonance absorber, the following three assumptions are used:

1) Non-resonance isotopes have negligible absorption and a constant potential scattering cro ss section for the resonance energy range.

2) Resonance isotopes have resonance absorption $\left(\sigma_{a i}(u)\right)$ and scattering $\left(\sigma_{r s, i}(u)\right)$ cross sect ions in addition to the potential scattering cross section $\left(\sigma_{p i}\right)$. However, apart from the re sonance energy region, these resonance absorption and scattering cross sections are negli gible.

3) The resonances are so well-separated that the flux between them has its constant asympto tic value (i.e., set to 1 ).

With these assumptions, the slowing down equation at resonance energy region, and away from fission sources is given by

$$
\begin{aligned}
& \Sigma(u) \phi(u)=Q(u), \\
& Q(u)=\sum_{i} \int_{u-\Delta_{i}}^{u} \Sigma_{s i}\left(u^{\prime}\right) \phi\left(u^{\prime}\right) \frac{\exp \left(u^{\prime}-u\right)}{1-\alpha_{i}} d u^{\prime},
\end{aligned}
$$

where 


$$
\begin{aligned}
& \Sigma_{x i}=N_{i} \sigma_{x i}, \\
& \Sigma_{s i}(u)=\Sigma_{p i}+\Sigma_{r s, i}(u), \\
& \sum_{i}(u)=\Sigma_{s i}(u)+\Sigma_{a i}(u), \\
& \Sigma(u)=\sum_{i} \Sigma_{i}(u), \\
& \alpha_{i}=\left(A_{i}-1\right)^{2} /\left(A_{i}+1\right)^{2}, \\
& \Delta_{i}=-\ln \left(\alpha_{i}\right) .
\end{aligned}
$$

In eq. (3.4.3), $\Sigma(u)$ and $N$ are the total macroscopic cross section and atomic number density, respectively and $A_{i}$ and $\Delta_{i}$ are the atomic mass and the maximum lethargy gain per collision with isotope i, respectively. $1-\alpha_{i}$ is the maximum fractional energy loss per collision with isotope $i$.

In a homogeneous system, for a given composition the self-shielded cross section can be calculated using equations (3.4.1) through (3.4.3) and the corresponding background cross section can be calculated using eq. (3.4.5).

$$
\sigma_{b}=\frac{\sum_{i=\text { all }} N_{i} \lambda_{i} \sigma_{i, p}}{N_{R}} .
$$

Different background cross sections can be obtained by using various composition mixtures (typically a mixture of ${ }^{1} \mathrm{H}$ and target resonance nuclide), creating a self-shielded cross-section table as a function background cross section. Therefore, for a given composition, the corresponding background cross section can be easily calculated using eq. (3.4.5) and the selfshielded cross section can be read from the table directly.

\section{$\underline{3.5 \text { Resonance self-shielded data by heterogeneous models }}$}

In a heterogeneous system, for a given composition and geometry the self-shielded cross section can also be calculated using equations (3.4.1) through (3.4.3). However, since there is a leakage effect in equation (3.1.1); equation (3.3.1) cannot be used to obtain the corresponding background cross section. Thus, the equivalence theory between the infinite homogeneous and heterogeneous problems was devised by introducing an equivalence cross section $\left(\Sigma_{e}\right)$ as follows:

$$
\Sigma_{b}=\lambda \Sigma_{p} \rightarrow \Sigma_{b}=\lambda \Sigma_{p}+\Sigma_{e}
$$

The balance equation with an equivalence theory and an elimination of the scattering resonance $\left(\lambda \Sigma_{r s}\right)$ thus becomes:

$$
\left(\Sigma_{a}+\lambda \Sigma_{p}+\Sigma_{e}\right) \phi=\lambda \Sigma_{p}+\Sigma_{e}
$$




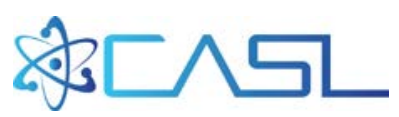

The background cross section is not a physical quantity but an artificial one used to retrieve the correct self-shielded cross section at a given composition and geometry. It is only important to be consistent in the procedures between its generation and its use. Therefore, the elimination of the scattering resonance is possible as long as consistency is maintained. In a heterogeneous system, the self-shielded scalar flux can be obtained by solving eq. (3.2.1) with a self-shielded absorption cross section obtained by solving eq. (3.1.1). The corresponding background cross section is obtained as follows:

$$
\sigma_{g, b}=\frac{\Sigma_{g, b}}{N_{R}}=\frac{\sum_{i} N_{i} \lambda_{i} \sigma_{i, g, p}+\Sigma_{g, e}}{N_{R}}=\frac{\sigma_{g, a} \phi_{g}}{1-\phi_{g}} .
$$

The following is a procedure to obtain the self-shielded cross section table.

(a) Compute the pointwise slowing down calculations, eq. (3.1.1), by using CENTRM/PMC for the heterogeneous models.

(b) Edit various MG self-shielded cross sections including absorption $\left(\sigma_{g, a}\right)$, fission $\left(\sigma_{g, f}\right)$ and scattering $\left(\sigma_{g, s}\right)$.

(c) Solve the MG fixed source eq. (3.5.4) with $\sigma_{g, a}$ from step (b) to obtain the scalar flux $\left(\phi_{g}\right)$.

$$
\hat{\Omega} \cdot \nabla \psi_{g, k}+\sum_{i}\left(\Sigma_{i, g, a}^{k}+\lambda_{i, g} \Sigma_{i, p}^{k}\right) \psi_{g, k}(\hat{\Omega})=\sum_{i} \lambda_{i, g} \Sigma_{i, p}^{k}
$$

(d) Obtain the corresponding background cross section $\left(\sigma_{g, b}\right)$ using eq. (3.5.3) for the heterogeneous model.

(e) Repeat the procedures (a) through (d) by changing the geometry and composition configurations to obtain different background cross sections.

(f) Add infinite dilution cross sections and complete the self-shielded cross section tables as a function of background cross section for $\sigma_{g, a}, \sigma_{g, f}$ and $\sigma_{g, s}$.

(g) Repeat the above procedures for various temperatures.

In eq. (3.4.1) the numerator is defined as a resonance integral (RI) with the flux and cross section a function of lethargy instead of Energy. In reference [Sta83], the self-shield cross section is approximated using the background cross section $\left(\sigma_{b}\right)$ and the resonance integral:

$$
\sigma_{g, a}\left(\sigma_{b}\right)=\frac{R_{g, a}\left(\sigma_{b}\right)}{1-R_{g, a}\left(\sigma_{b}\right) / \sigma_{b}}
$$

and

$$
v \sigma_{g, f}\left(\sigma_{b}\right)=\frac{R_{g, v f}\left(\sigma_{b}\right)}{1-R_{g, a}\left(\sigma_{b}\right) / \sigma_{b}}
$$


where $\sigma_{g, a}$ is an absorption cross section, $\sigma_{g, f}$ a fission cross section, $v$ the number of neutrons released from a fission, and $R_{g, a}$ and $R_{g, v f}$ are absorption and $v^{*}$ fission resonance integrals, respectively. Using equations (3.5.5) and (3.5.6), the self-shield cross section table can be converted into the resonance integral table.

Various background cross sections can be achieved by changing geometrical and compositional configurations as shown in Table 3.5.1.

Table 3.5.1 Example of variations for ${ }^{238} \mathrm{U}$

\begin{tabular}{|c|c|c|c|c|c|c|c|c|c|c|c|c|c|c|c|c|c|}
\hline \multicolumn{2}{|c|}{ Case } & 1 & 2 & 3 & 4 & 5 & 6 & 7 & 8 & 9 & 10 & 11 & 12 & 13 & 14 & 15 & 16 \\
\hline \multirow{3}{*}{ Volume } & Fuel & 1.0 & 1.0 & 1.0 & 1.0 & 1.0 & 1.0 & 1.0 & 1.0 & 1.0 & 1.0 & 1.0 & 1.0 & 1.0 & 1.0 & 1.0 & 1.0 \\
\hline & Clad & 1.0 & 1.0 & 1.0 & 1.0 & 1.0 & 1.0 & 1.0 & 1.0 & 1.0 & 1.0 & 1.0 & 1.0 & 1.0 & 1.0 & 1.0 & 1.0 \\
\hline & Mod & 1.0 & 1.0 & 1.0 & 1.0 & 1.0 & 2.0 & 5.0 & 5.0 & 5.0 & 5.0 & 5.0 & 5.0 & 5.0 & 5.0 & 5.0 & 5.0 \\
\hline \multirow{3}{*}{ Fuel } & ${ }^{235} \mathrm{U}$ & 1.0 & 1.0 & 1.0 & 1.0 & 1.0 & 1.0 & 1.0 & 0.5 & 0.25 & 0.125 & 0.0625 & 0.03125 & 0.01 & \begin{tabular}{|l|}
0.01 \\
\end{tabular} & 0.01 & 0.01 \\
\hline & ${ }^{238} \mathrm{U}$ & 1.0 & 1.0 & 1.0 & 1.0 & 1.0 & 1.0 & 1.0 & 0.5 & 0.25 & 0.125 & 0.0625 & 0.03125 & 0.01 & 0.001 & $1.0 \mathrm{E}-6$ & $1.0 \mathrm{E}-7$ \\
\hline & ${ }^{16} \mathrm{O}$ & 1.0 & 1.0 & 1.0 & 1.0 & 1.0 & 1.0 & 1.0 & 0.5 & 0.25 & 0.125 & 0.0625 & 0.03125 & 0.01 & 0.01 & 0.01 & 0.01 \\
\hline \multirow{2}{*}{$\mathrm{H}_{2} \mathrm{O}$} & ${ }^{1} \mathrm{H}$ & $2.5 \mathrm{E}-3$ & 0.2 & 0.5 & 0.75 & 1.0 & 1.0 & 1.0 & 1.0 & 1.0 & 1.0 & 1.0 & 1.0 & \begin{tabular}{|l|}
1.0 \\
\end{tabular} & 1.0 & 1.0 & 1.0 \\
\hline & ${ }^{16} \mathrm{O}$ & 2.5E-3 & 0.2 & 0.5 & 0.75 & 1.0 & 1.0 & 1.0 & 1.0 & 1.0 & 1.0 & 1.0 & 1.0 & 1.0 & 1.0 & 1.0 & 1.0 \\
\hline
\end{tabular}

\subsection{Resonance self-shielded data by Monte Carlo code}

Conceptually this procedure is almost same with the deterministic procedure introduced in Sections 3.4 and 3.5. The only difference is to perform Monte Carlo CE calculations to edit MG self-shielded cross sections instead of performing pointwise slowing down calculation with a deterministic transport code. Either eigenvalue or fixed source Monte Carlo calculations can be performed.

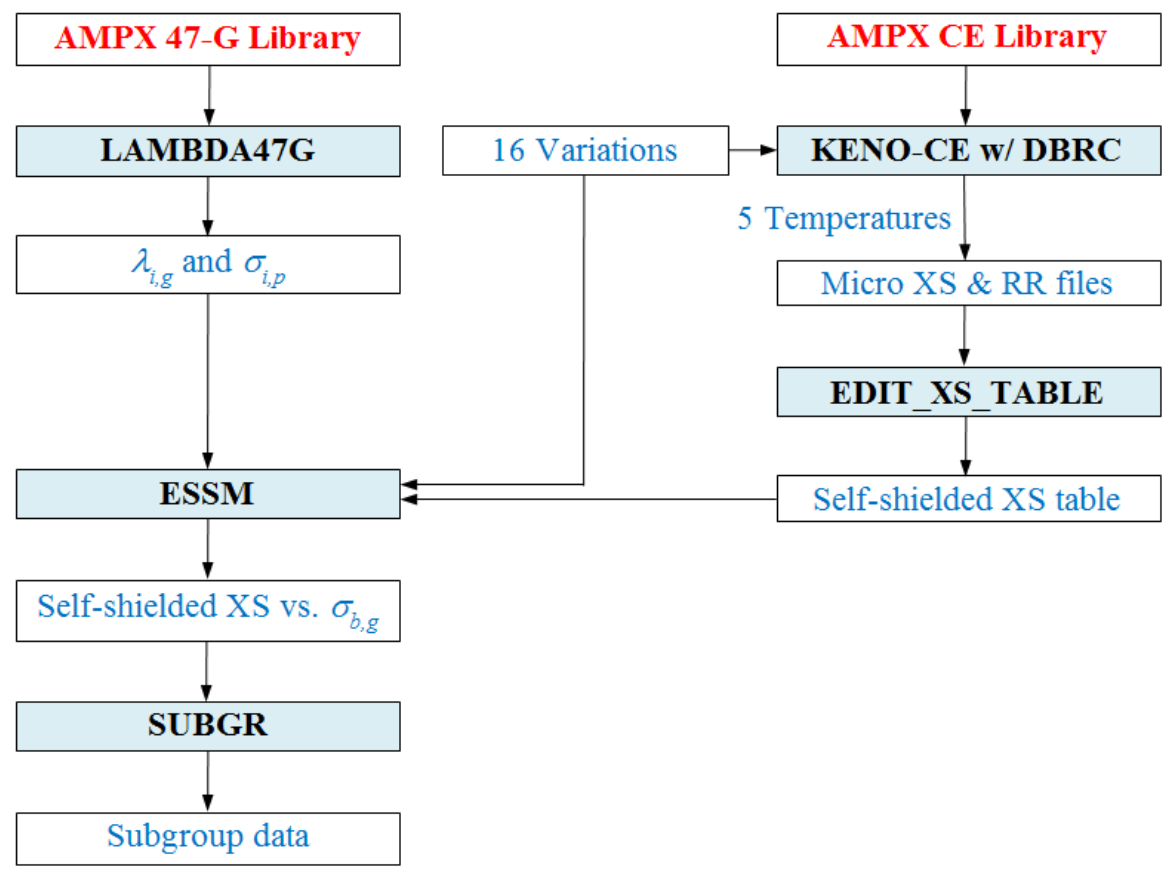


Figure 3.6.1 Procedure to generate resonance data with epithermal upscattering

No deterministic slowing down program is available to consider epithermal upscattering ( $>5.0$ $\mathrm{eV}$ ). Therefore the Bondarenko f-factor (self-shielded cross section) tables must be generated by performing the continuous energy Monte Carlo calculations and evaluating the corresponding background cross sections [Kim03]. The F-factor tables can be generated by using either homogeneous or heterogeneous models. The key feature of this procedure is to perform the Monte Carlo calculations to obtain MG self-shielded cross sections instead of solving deterministic PW slowing down calculations. In this study, the KENO CE calculations were performed to edit effective microscopic cross sections rather than the CENTRM slowing down calculations. The corresponding background cross sections can be obtained by the same method described in Sections 3.4 and 3.5. The Bondarenko F-factors have been obtained by performing the KENO CE calculations without and with the Doppler Broadening Rejection Correction (DBRC) [Bec10] option to consider the resonance upscattering effect. Figure 3.6.1 provides the procedure to generate resonance data with epithermal upscattering by using KENO-CE.

\section{$\underline{\text { 3.7 Unresolved resonance self-shielded data }}$}

In the conventional MG procedure, the Bondarenko f-factor tables at the URR energy groups are generated by using a narrow resonance approximation with a J-function method [Lee11] for which arbitrary background cross-sections are provided. The background cross-section is estimated for the specified composition and geometry, and the self-shielded 1-D cross-sections are read from the f-factor table. This procedure introduces some bias in the self-shielded crosssections at the URR groups. We are going to solve this problem by improving the overall procedure including a new generation of the f-factor tables for the URR groups.

Typically narrow resonance approximation could be a good solution for the URR energy range in which PW neutron spectrum could be approximated in terms of total and background crosssections. Since the probability tables are provided at the URR energy range which include crosssection levels and weights, multi-group self-shielded cross-section tables (f-factor table) could be obtained by using a narrow resonance approximation with probability table as follows:

$$
\begin{gathered}
\sigma_{x, g}\left(\sigma_{0}\right)=\frac{\int_{g} \sum_{m} p^{m} \sigma_{x}^{m}(E) \phi^{m}\left(E, \sigma_{0}\right) d E}{\int_{g} \sum_{m} p^{m} \phi^{m}\left(E, \sigma_{0}\right) d E}, \\
\phi^{m}\left(E, \sigma_{0}\right)=\frac{\sigma_{0}}{\sigma_{t}^{m}(E)+\sigma_{0}} W(E),
\end{gathered}
$$

where

$\sigma_{0}=$ background cross-section,

$\sigma_{x}^{m}=$ a cross-section level of the level $m$ and reaction $x$,

$\sigma_{t}^{m}=$ a total cross-section level of the level $m$, 


$$
\begin{aligned}
& p^{m}=\text { a probability of the level } m, \\
& \sigma_{x, g}=\text { a self-shielded cross-section of reaction } x
\end{aligned}
$$

\section{$\underline{3.8 \text { Self-shielded data for within-group elastic scattering }}$}

The AMPX MG library does include temperature independent elastic scattering matrix which results in temperature bias when performing self-shielding calculation based on Bondarenko approach. Recently new MT=2022 has been added to consider removal correction to generate temperature dependent elastic scattering matrix. DECLIB has been modified to generate temperature dependent temperature dependent elastic scattering matrix by using pre-obtained temperature dependent background cross sections.

The new MT=2022 is for within-group cross section ( $\sigma_{g}^{\text {within }}$ ) for elastic scattering which includes Bondarenko F-factors and 1D data. If background cross section is determined by ESSM or subgroup method, total elastic and within-group elastic cross sections can be obtained through interpolation. Temperature dependent elastic scattering matrix at temperature $T$ can be obtained by using the following equations.

$$
\begin{gathered}
\tilde{\sigma}_{g g}^{\text {elastic }}(T)=\sigma_{g}^{\text {within }}(T) . \\
\tilde{\sigma}_{g g^{\prime}}^{\text {elastic }}(T)=\sigma_{g g^{\prime}}^{\text {elastic }}\left(T_{0}\right) \cdot \frac{\sigma_{g}^{\text {elastic }}(T)-\sigma_{g}^{\text {within }}(T)}{\sum_{g^{\prime}} \sigma_{g g^{\prime}}^{\text {elastic }}\left(T_{0}\right)-\sigma_{g g}^{\text {elastic }}\left(T_{0}\right)} .
\end{gathered}
$$

The DECLIB program has been modified to estimate temperature dependent elastic scattering matrix by using eqs. (3.8.1) and (3.8.2) with the nuclide-wise background cross sections. 


\section{RESONANCE SELF-SHIELDING METHODS}

\section{$\underline{4.1 \text { Subgroup method }}$}

Figure 4.1.1 shows the coarse energy group including resonances. Effective self-shielded cross section for this group can be obtained by the following flux weighting:

$$
\sigma_{x, g}=\frac{\int_{\Delta u_{g}} \sigma_{x}(u) \phi(u) d u}{\int_{\Delta u_{g}} \phi(u) d u},
$$

where $\sigma_{x, g}$ denotes the effective cross section of reaction $x$ at the energy group $g, u$ is lethargy, and $\phi(u)$ the continuos or pointwise sclar flux. Continuous scalar flux can be obtained by solving the slowing down equation with the point-wise cross sections.

In the subgroup method, the resonances are divided by the subgroup levels and the corresponding probability for each subgroup level $\left(\sigma_{x n}\right)$ is defined as the subgroup weight $\left(w_{x n}\right)$ as shown in Figure 4.1.1. [Hel03] Therefore, eq. (4.1.1) can be approximated with removing the coarse group index as follows:

$$
\sigma_{x}=\frac{\sum_{n} w_{x n} \sigma_{x n} \phi_{n}}{\sum_{n} w_{a n} \phi_{n}},
$$

where the summation of the subgroup weights is unity.

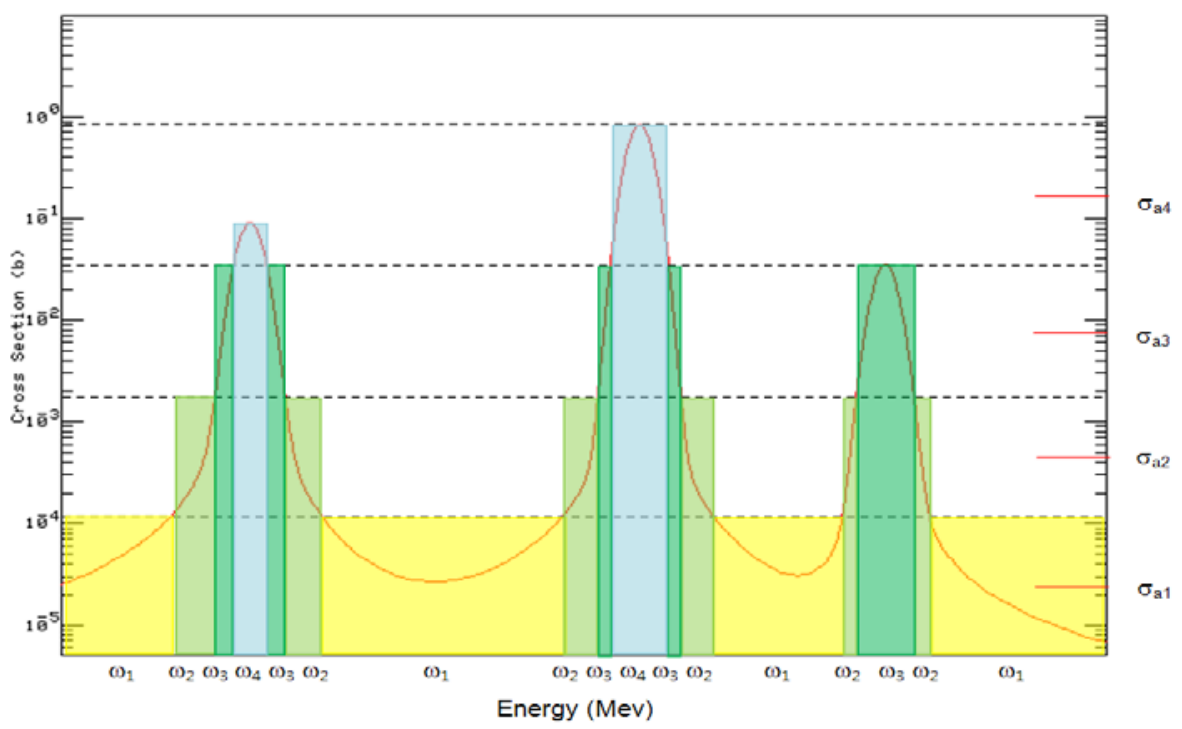

Figure 4.1.1 Resonances and subgroups 
In the transport lattice calculation, the final goal of the resonance treatment is to construct a procedure such that the self-shielded cross section estimated by eq. (4.1.2) will be identical to the self-shielded cross section by eq. (4.1.1). The self-shielded sclar flux $\phi_{n}$ in eq. (4.1.2) should be estimated from the fixed source transport calculation. If there is only one resonant nuclide, the fixed source transport equation will be

$$
\hat{\Omega} \cdot \nabla \psi_{n}+\left(N_{r} \sigma_{a n}+\sum_{i} \lambda_{i} \Sigma_{p}^{i}\right) \psi_{n}=\sum_{i} \lambda_{i} \Sigma_{p}^{i}
$$

and

$$
\phi_{n}=\int \psi_{n} d \hat{\Omega}
$$

where $N_{r}$ denotes the particle number density for the resonant nuclide, $\Sigma_{p}$ a potential cross section, and $\psi_{n}$ an angular flux. Equivalence theory enforces that the self-shielded scalar flux is expressed with the absorption $\left(\sigma_{a n}\right)$ and the background cross sections $\left(\sigma_{b n}\right)$.

$$
\phi_{n}=\frac{\sigma_{b n}}{\sigma_{a n}+\sigma_{b n}} .
$$

Typically the background cross section in the heterogeneous model is divided into two parts as follows:

$$
\sigma_{b n}=\lambda \sigma_{p}+\sigma_{e n}\left(\sigma_{a n}\right)
$$

where $\lambda$ denotes an intermediate resonance parameter, $\sigma_{p}$ a potential cross section, and $\sigma_{e n}$ an equivalence cross section. By using eq. (4.1.5), eq. (4.1.2) can be written as follows:

$$
\sigma_{x}=\frac{\sum_{n} w_{x n} \sigma_{x n} \frac{\sigma_{b n}}{\sigma_{a n}+\sigma_{b n}}}{1-\sum_{n} w_{a n} \frac{\sigma_{a n}}{\sigma_{a n}+\sigma_{b n}}} .
$$

Therefore, if the subgroup levels and weights are given for a certain nuclide, the effective selfshielded cross section can be obtained by estimating the corresponding background cross sections $\left(\sigma_{b n}\right)$ in the lattice calculation. Since the equivalence cross section is not sensitive to the absorption cross section, but to the geometrical configuration, $\sigma_{b n}$ is often approximated by a single background cross section $\sigma_{b}$. Eq. (4.1.7) can be written as follows: 


$$
\sigma_{x}=\frac{\sum_{n} w_{x n} \sigma_{x n} \frac{\sigma_{b}}{\sigma_{a n}+\sigma_{b}}}{1-\sum_{n} w_{a n} \frac{\sigma_{a n}}{\sigma_{a n}+\sigma_{b}}} .
$$

If there are several resonant nuclides, there will be a resonant interference between the resonant nuclides. Since this interference will affect on the self-shielded scalar flux, eq. (4.1.8) can be written as follows:

$$
\sigma_{x}=\frac{\sum_{n} w_{x n} \sigma_{x n} \frac{\sigma_{b}}{\sigma_{a n}+\hat{\sigma}_{a}+\sigma_{b}}}{1-\sum_{n} w_{a n} \frac{\sigma_{a n}}{\sigma_{a n}+\hat{\sigma}_{a}+\sigma_{b}}},
$$

where $\hat{\sigma}_{a}$ is the total absorption cross section of the other resonant nuclides. The effective selfshielded cross sections are estimated iteratively.

\section{$\underline{4.2 \text { Subgroup data generation }}$}

The resonance integral $\left(R I_{x}\right)$ is defined as the numerator of eq. (4.1.1), and the resonance integral divided by lethargy width is also called resonance integral $\left(R_{x}\right)$. The resonance integral can be written by using the subgroup weights and levels from eq. (4.1.7) as follows:

$$
R_{x}=\sum_{n} w_{n} \sigma_{x n} \frac{\sigma_{b n}}{\sigma_{a n}+\sigma_{b n}} \approx \sum_{n} w_{n} \sigma_{x n} \frac{\sigma_{b}}{\sigma_{a n}+\sigma_{b}}
$$

Since the denominator of eq. (4.1.1) can be understood as a scalar flux ( $\phi$ ) for the coarse energy group, eq. (4.1.1) can be rewritten as follows:

$$
\sigma_{x}=\frac{R_{x}}{\phi} .
$$

As described in Section 3.5, the constituent compositions and the geometrical configurations are varied to obtain various background cross sections to complete the resonance integral table. Index ' $k$ ' for the variation cases can be added to eq. (4.2.2).

$$
\sigma_{x, k}=\frac{R_{x, k}}{\phi_{k}} \quad(k=1,2, \ldots, K) .
$$

The resonance integral $\left(R_{x, k}\right)$ can be estimated directly from the IRFFACTOR program for the variation cases. The subgroup levels and weights can be obtained from eqs. (4.2.1) by the least 
square fitting to minimize the difference between the original resonance integral and the reconstructed resonance integral using eq. (4.2.1).

Subgroup levels are arbitrary given and the corresponding subgroup weights are obtained by minimizing the following function $f$.

$$
\begin{aligned}
& f\left(w_{1}, \ldots, w_{K}\right)=\sum_{k} \frac{1}{R_{x, k}^{2}}\left(R_{x, k}-\sum_{n} w_{x n} \sigma_{x n, k} \frac{\sigma_{b n, k}}{\sigma_{a n, k}+\sigma_{b n, k}}\right)^{2} . \\
& f\left(w_{1}, \ldots, w_{K}\right)=\sum_{k} \frac{1}{R_{x, k}^{2}}\left(R_{x, k}-\sum_{n} w_{x n} \sigma_{x n, k} \frac{\sigma_{b, k}}{\sigma_{a n, k}+\sigma_{b, k}}\right)^{2} .
\end{aligned}
$$

Subgroup levels are to be automatically adjusted to minimize the difference between the original resonance integral and the reconstructed resonance integral using the subgroup levels and weights. The resonance integral table from the IRFFACTOR calculation includes the background cross sections as a function of absorption cross section at each variation $k$ to cover all the subgroup levels. Although the subgroup levels are varied at iterations, the corresponding background cross section $\left(\sigma_{b n, k}\right)$ can be obtained through the interpolation of the given table. SUBGR includes two options to generate the subgroup weights and levels by using eqs. (4.2.4) and (4.2.5) which use the level dependent and constant background cross sections, respectively.

When the resonance interference is neglected, errors in estimating the effective self-shielded cross section come mainly from the subgroup levels and weights themselves, and the scalar flux estimation. In the real application the self-shielded scalar flux is estimated by the following equation.

$$
\hat{\phi}_{k}=1-\sum_{n} w_{a n} \frac{\sigma_{a n, k}}{\sigma_{a n, k}+\sigma_{b n, k}},
$$

where the subgroup weights and levels are given and the corresponding background cross sections $\left(\sigma_{b n, k}\right)$ are obtained by the fixed source transport calculations of the transport lattice code using eq. (4.1.3). The difference between the scalar flux $\left(\phi_{k}\right)$ in eq. (4.2.3) and the scalar $\operatorname{flux}\left(\hat{\phi}_{k}\right)$ in eq. (4.2.6) causes a difference in the effective self-shielded cross section.

In order to remove this error a new method [Joo09] has been proposed recently to generate the subgroup weights, in which the subgroup weights are to be estimated to conserve the selfshielded cross sections as follows:

$$
f\left(w_{1}, \ldots, w_{K}\right)=\sum_{k} \frac{1}{R_{x, k}^{2}}\left(\hat{R}_{x, k}-\sum_{n} w_{x n} \sigma_{x n, k} \frac{\sigma_{b n, k}}{\sigma_{a n, k}+\sigma_{b n, k}}\right)^{2},
$$

where

$$
\hat{R}_{x, k}=\sigma_{x, k} \hat{\phi}_{k}=\sigma_{x, k}\left(1-\sum_{n} w_{a n} \frac{\sigma_{a n, k}}{\sigma_{a n, k}+\sigma_{b n, k}}\right) .
$$




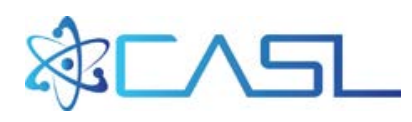

\section{$\underline{4.3 \text { Subgroup method for non-uniform temperature distribution }}$}

In a heterogeneous system, the self-shielded resonance cross sections are estimated from the selfshielded scalar fluxes obtained by the following fixed source transport equation.

$$
\hat{\Omega} \cdot \nabla \psi_{g, m}+\sum_{i}\left(\Sigma_{i, a, g}^{m}+\lambda_{i, g} \Sigma_{i, p}\right) \psi_{g, m}(\hat{\Omega})=\sum_{i} \lambda_{i, g} \Sigma_{i, p}
$$

where subscript $m$ denotes a problem case with different absorption cross section levels at energy group $g$. In eq. (4.3.1), $\Sigma_{i, a, g}$ and $\Sigma_{i, p}$ denote macroscopic absorption and potential cross sections of nuclide $i$, respectively, and $\lambda_{i, g}$ intermediate resonance parameter.

Eq. (4.3.1) should be modified for the resonance transport calculations involving non-uniform temperature distribution in which the macroscopic absorption cross sections should include the temperature distribution as follows:

$$
\sum_{i, a, g}^{m}=N_{i} \sigma_{i, a, g}^{m}\left(T_{a v e .}\right) \frac{\sigma_{i, a, g}^{m}(T)}{\sigma_{i, a, g}^{m}\left(T_{\text {ave. }}\right)} \approx N_{i} \sigma_{i, a, g}^{m}\left(T_{\text {ave. }}\right) f_{i, a, g}(T),
$$

where $T$ and $T_{\text {ave. }}$ are local and volume-averaged temperatures, respectively. While the function $f(T)$ was approximated by the following equation [Wem07].

where

$$
f_{i, a, g}(T)=\frac{R_{i, a, g}\left(T, \sigma_{p}\right)}{R_{i, a, g}\left(T_{a v e .}, \sigma_{p}\right)} \cdot \frac{\sigma_{p}-R_{i, a, g}\left(T_{a v e .}, \sigma_{p}\right)}{\sigma_{p}-R_{i, a, g}\left(T, \sigma_{p}\right)},
$$

$$
\sigma_{p} \approx \frac{\sum_{j=\text { all }} N_{j} \lambda_{j, g} \sigma_{j, p}}{N_{i}}
$$

$N_{i}$ is the particle number density of nuclide $i$, and $R_{i, a, g}$ resonance integral. Eq. (4.3.4) can be rewritten without any approximation as follows:

$$
\sigma_{b, g}^{m}=\frac{\sum_{j=a l l} N_{j} \lambda_{j, g} \sigma_{j, p}+\sum_{e, g}^{m}}{N_{i}}
$$

Therefore the explicit equation will be

$$
f_{i, a, g}^{m}(T)=\frac{\sigma_{i, a, g}^{m}(T)}{\sigma_{i, a, g}^{m}\left(T_{a v e}\right)}=\frac{R_{i, a, g}\left(T, \sigma_{b, g}^{m}\right)}{R_{i, a, g}\left(T_{a v e .}, \sigma_{b, g}^{m}\right)} \cdot \frac{\sigma_{b, g}^{m}-R_{i, a, g}\left(T_{a v e .}, \sigma_{b, g}^{m}\right)}{\sigma_{b, g}^{m}-R_{i, a, g}\left(T, \sigma_{b, g}^{m}\right)} .
$$

When performing a MPACT calculation with T-H feedback, $\Sigma_{e, g}^{m}$ can be obtained from the previous outer iteration. 
Another better approximation is to get the correction factor from the previous step as follows:

$$
\sum_{i, a, g}^{m(\ell+1)}=N_{i} \sigma_{i, a, g}^{m}\left(T_{\text {ave. }}\right) \frac{\sigma_{i, a, g}^{m}(T)}{\sigma_{i, a, g}^{m}\left(T_{\text {ave. }}\right)} \approx N_{i} \sigma_{i, a, g}^{m}\left(T_{\text {ave. }}\right) \frac{\sigma_{i, a, g}^{(\ell)}(T)}{\sigma_{i, a, g}^{(\ell)}\left(T_{\text {ave. }}\right)} .
$$

\subsection{Embedded self-shielding method (ESSM)}

ESSM [Hon11, Wil12] has been developed for the resonance self-shielding calculation which requires the fixed source transport calculations in estimating background cross sections. When estimating resonance self-shielded cross section by ESSM, absorption cross sections $\left(\Sigma_{i, a, g}\right)$ at fuel and cladding are iteratively determined by solving the fixed source transport eq. (4.4.1).

$$
\begin{gathered}
\hat{\Omega} \cdot \nabla \varphi_{g}\left(\hat{\Omega}_{m}\right)+\sum_{j}\left(\Sigma_{j, a, g}+\lambda_{j, g} \Sigma_{j, p}\right) \varphi_{g}\left(\hat{\Omega}_{m}\right)=\sum_{j} \lambda_{j, g} \Sigma_{j, p}, \\
\hat{\Omega} \cdot \nabla \varphi_{g}\left(\hat{\Omega}_{m}\right)+\Sigma_{t, g} \varphi_{g}\left(\hat{\Omega}_{m}\right)=S_{g},
\end{gathered}
$$

where $\varphi_{g}$ is an angular flux of group $g, \Sigma_{j, a, g}$ a macroscopic absorption cross section of nuclide $j, \Sigma_{j, p}$ a macroscopic potential cross section and $\lambda_{j, g}$ an intermediate resonance parameter. The corresponding macroscopic and microscopic background cross sections can be obtained by using the following eq. (4.4.3).

$$
\Sigma_{i, b, g}=\frac{\Sigma_{i, a, g} \phi_{i, g}}{1-\phi_{i, g}} \rightarrow \sigma_{i, b, g}^{j}=\frac{\Sigma_{i, b, g}}{N_{i, j}}
$$

Self-shielded absorption cross sections for each nuclide can be read from the Bondarenko Ffactor tables in the AMPX MG library [Dun02], and the absorption cross sections are utilized in updating $\Sigma_{j, a, g}$. And then the same ESSM calculation will be performed. This iteration procedure will be continued until the macroscopic background cross sections $\left(\Sigma_{i, b, g}\right)$ are converged.

There are several issues associated with ESSM as follows:

- Counter influence between cladding and fuel in ESSM

- ESSM with correction factors for non-uniform temperature distribution

The above issues are discussed in detail and probable solutions are proposed for some issues. 


\section{GENERATION OF MISCELLANEOUS DATA}

\section{$\underline{5.1 \text { Transport cross sections for }}{ }^{\underline{1}} \underline{\mathrm{H}}$}

The MOC eigenvalue calculation with high order $\left(\geq \mathrm{P}_{2}\right)$ scattering requires 2 times longer computing time compared to the $\mathrm{P}_{0}$ calculation. Typically most of transport lattice codes are utilizing out-scattering based transport corrected $\mathrm{P}_{0}\left(\mathrm{TCP}_{0}\right)$ scattering matrix in which diagonal terms of $\mathrm{P}_{0}$ scattering matrix are subtracted by total $\mathrm{P}_{1}$ scattering cross sections. The $\mathrm{P}_{1}$ corrected $\mathrm{P}_{0}$ scattering matrix for ${ }^{1} \mathrm{H}$ may include negative value in the diagonal components which causes negative flux resulting in convergence error. Another issue of out-scattering based transport correction does show significant global power tilt for whole core problems including reflector. Therefore a proper transport correction method is needed to have reasonable neutron leakage as high order scattering is considered and to guarantee no negative flux.

The Neutron Leakage Conservation method $\left(\mathrm{NLCP}_{0}\right)$ [Her13] used in the CASMO series for long time has been used to generate transport cross sections for ${ }^{1} \mathrm{H}$. The goal of $\mathrm{NLCP}_{0}$ is to obtain diffusion coefficients to have same neutron leakage as obtained in the high order scattering transport calculation as follows:

$$
\hat{\Omega} \cdot \nabla \varphi_{g}(\hat{\Omega})=-D_{g} \nabla^{2} \phi_{g}
$$

When we have a 1D model, neutron leakages at the specified surfaces can be obtained from the high order scattering transport calculation.

$$
\text { Leakage }=J_{\text {right }}-J_{\text {left }} \text {. }
$$

We can determine diffusion coefficients to have same leakage in the diffusion calculation by using the following derivation.

$$
\begin{gathered}
-D \nabla^{2} \phi+\Sigma_{a} \phi=\frac{1}{k_{\text {eff }}} v \Sigma_{f} \phi . \\
\nabla^{2} \phi+B^{2} \phi=0 \rightarrow \frac{\partial^{2}}{\partial x^{2}} \phi(x)+B^{2} \phi(x)=0 . \\
\phi(x)=\cos (B x) . \\
J_{\text {right }}-J_{\text {left }}=\int_{0}^{W} d x\left(-D \frac{\partial^{2}}{\partial x^{2}} \phi(x)\right)=\int_{0}^{W} d x D B^{2} \phi(x)=D B^{2} \int_{0}^{W} d x \phi(x)=D B^{2} \hat{\phi} . \\
D=\frac{J_{\text {right }}-J_{\text {left }} .}{B^{2} \hat{\phi}} . \\
B_{\text {geom }}^{2}=\left(\frac{\pi}{W}\right)^{2} .
\end{gathered}
$$


When performing the multigroup transport calculation with high order scattering, it is required to check if the group-wise bucklings are constant independently upon energy group. This work can be completed by drawing normalized neutron spectrum shape for each group.

$$
B_{\text {geom }}^{2}=B^{2}=B_{g}^{2}
$$

We can obtain multi-group diffusion coefficients and transport cross sections.

$$
\begin{gathered}
D_{g}=\frac{J_{g, r i g h t}-J_{g, l e f t}}{B^{2} \hat{\phi}_{g}} . \\
\Sigma_{t r, g}=\frac{1}{3 D_{g}} .
\end{gathered}
$$

The computational model was from the reference paper [Her13] as follows:

- 1-D slab $100 \mathrm{~cm} \mathrm{w} /$ vacuum boundary, $0.005 \mathrm{~cm}$ mesh size

- All ${ }^{1} \mathrm{H}$ with $4.780 \mathrm{E}+23$ atom $/ \mathrm{cm}^{3}$

- 9 temperatures : 293.6, 350.0, 400.0, 450.0, 500.0, 550.0, 600.0, 650.0, 800.0 K

- Source : ${ }^{235} \mathrm{U}$ fission spectrum with buckled Cosine spatial distribution

This work can be done by using either continuous energy Monte Carlo or multigroup deterministic transport codes. A new 1D MOC code including high order scattering capability has been developed to generate transport corrected ${ }^{1} \mathrm{H}$ cross sections. Figure 5.1 .1 provides the 47-group transport correction factors for ${ }^{1} \mathrm{H}$ with various temperatures.

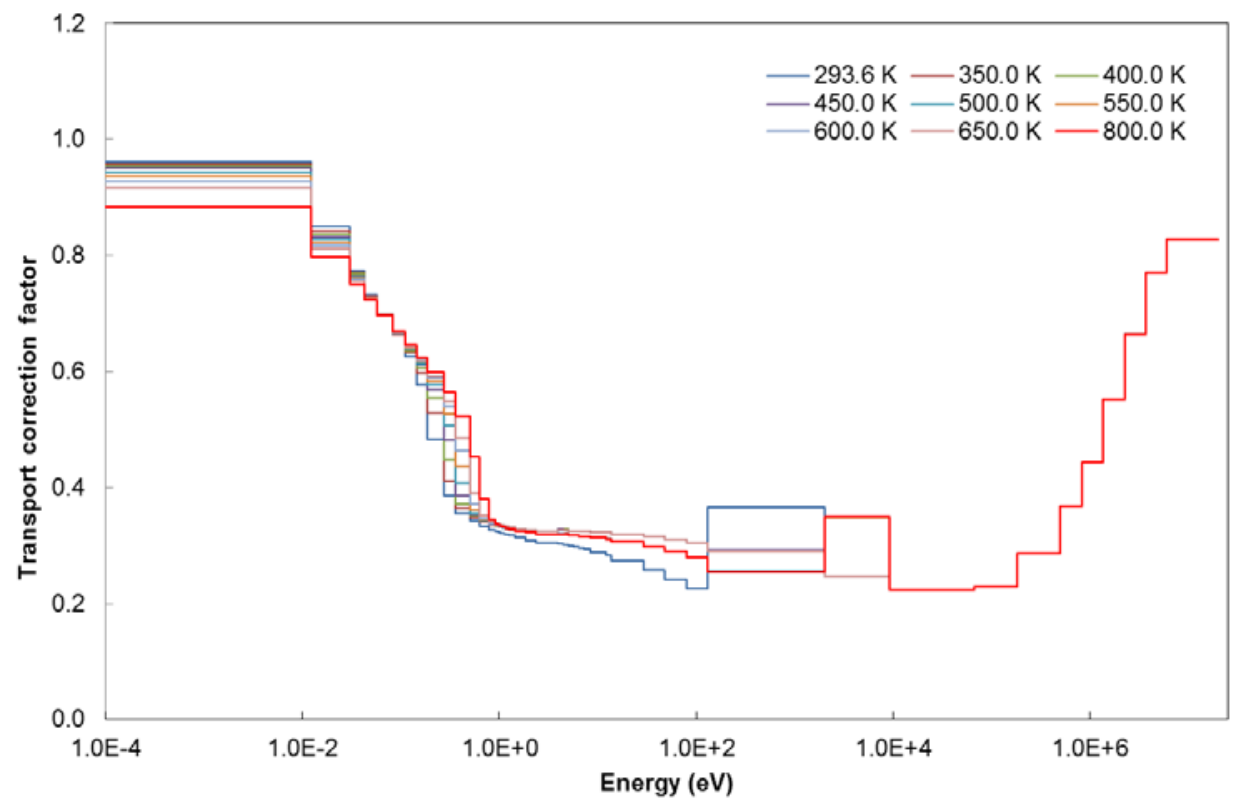

Figure 5.1.1 47-group transport correction factors for ${ }^{1} \mathrm{H}$ 


\subsection{Transient data}

Since the ORNL AMPX code package [Wia14] does not support to generate transient data such as decay constants and delayed neutron fractions, the NJOY program [Mac94] developed at LANL has been utilized to generate the transient data. Since there was no information for the ENDF/B nuclides including transient data, the NJOY input files were generated for all 37 heavy nuclides in the MPACT library. Tape 24 from the GROUPR module includes 47-group neutron data including transient data. A simple editing program has been developed to edit transient data such as decay constants and delayed neutron fractions. In order to obtain total number of neutrons released per fission to be used in obtaining delayed neutron fraction, typical 47-group PWR spectrum has been obtained from the VERA progression problem $1 \mathrm{~b}$ as shown Figure 5.2.1.

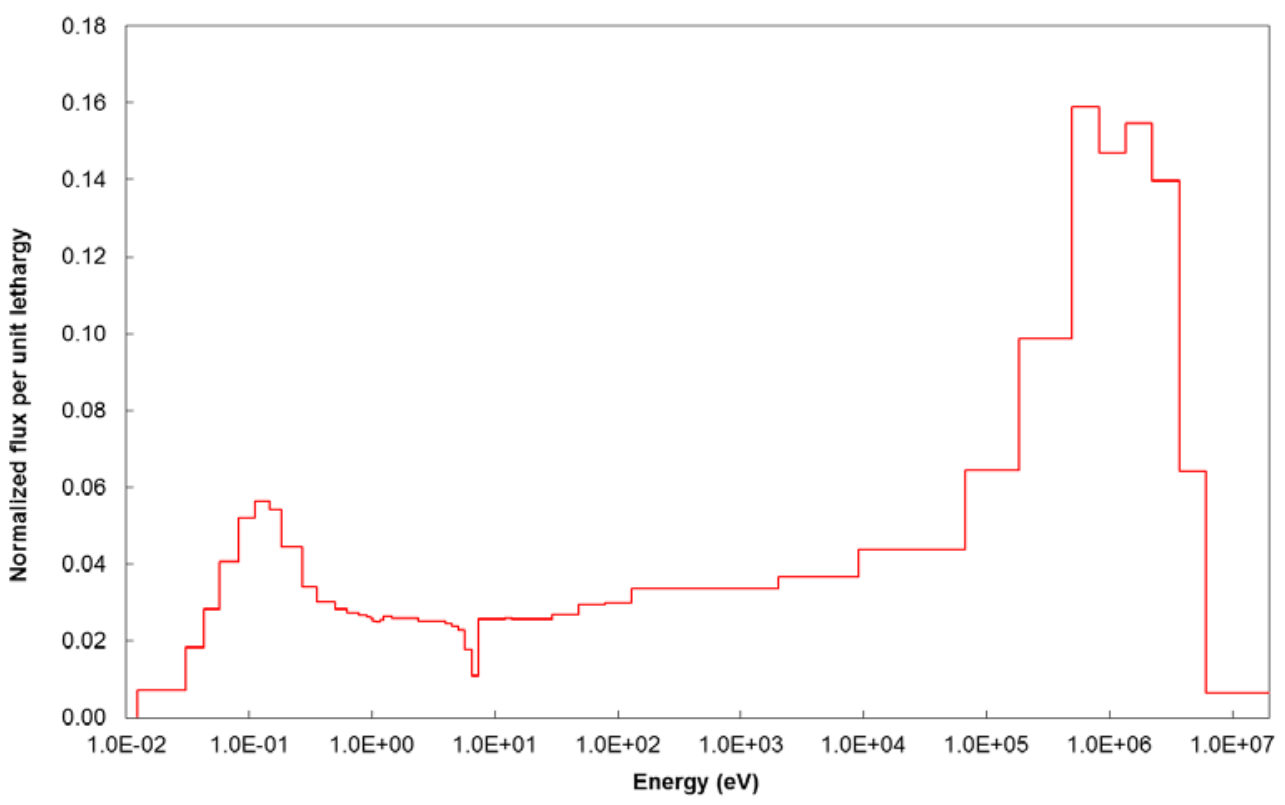

Figure 5.2.1 47-group weighting function

The ENDF/B-7.0 and 7.1 neutron data include transient data only for 21 nuclides. Figure 5.2.2 provides transient data including nuclide identification, total number of neutrons released per fission, decay constants and delayed neutron fractions for each nuclide.

\begin{tabular}{|c|c|c|c|c|c|}
\hline 90232 & $2.417105 \mathrm{E}+0 \odot$ & & & & \\
\hline 1. $240000 \mathrm{E}-\odot 2$ & $3.3400 \odot \odot E-\odot 2$ & $1.210000 \mathrm{E}-01$ & $3.21000 \odot \mathrm{E}-01$ & $1.2100 \odot \odot E+\odot \odot$ & $3.2900 \odot \odot E+\odot \odot$ \\
\hline $8.237550 \mathrm{E}-04$ & $2.839532 E-03$ & $3.391569 \mathrm{E}-\odot 3$ & $9.961879 E-03$ & $3.760367 \mathrm{E}-03$ & $1.827437 \mathrm{E}-03$ \\
\hline 91231 & $2.429466 \mathrm{E}+0 \odot$ & & & & \\
\hline 1. $240000 \mathrm{E}-\odot 2$ & $3.3400 \odot \odot E-\odot 2$ & $1.210000 \mathrm{E}-01$ & $3.210 \odot \odot \odot E-\odot 1$ & 1. $21000 \odot E+\odot \odot$ & $3.2900 \odot \odot E+\odot \odot$ \\
\hline $3.772028 \mathrm{E}-\odot 4$ & 1. $018876 E-03$ & $7.348728 E-04$ & $1.774840 \mathrm{E}-\odot 3$ & $4.798565 E-04$ & $1.831329 E-04$ \\
\hline 91233 & $2.429815 E+0 \odot$ & & & & \\
\hline 1.24000९E- 02 & $3.340000 \mathrm{E}-02$ & $1.210000 \mathrm{E}-01$ & $3.210000 \mathrm{E}-01$ & $1.210000 \mathrm{E}+0 \odot$ & $3.290000 \mathrm{E}+0 \odot$ \\
\hline $\begin{array}{r}7.746643 \mathrm{E}-04 \\
92233\end{array}$ & $\begin{array}{l}2.092295 \mathrm{E}-\odot 3 \\
2.500074 \mathrm{E}+\odot \odot\end{array}$ & 1.509164E-03 & $3.645136 \mathrm{E}-\odot 3$ & $9.855432 E-04$ & $3.761569 E-04$ \\
\hline
\end{tabular}




\begin{tabular}{|c|c|c|c|c|c|}
\hline $1.247938 \mathrm{E}-02$ & $3.227449 \mathrm{E}-02$ & $1.046448 \mathrm{E}-01$ & $2.941518 \mathrm{E}-01$ & $1.242443 \mathrm{E}+0 \odot$ & $1.022323 \mathrm{E}+01$ \\
\hline $2.567975 E-04$ & 7. . 701037E-๑4 & $5.537597 \mathrm{E}-04$ & $1.142208 \mathrm{E}-03$ & $2.146458 E-04$ & $2.239787 \mathrm{E}-05$ \\
\hline 92234 & $2.633240 \mathrm{E}+\odot \odot$ & & & & \\
\hline $1.248240 E-02$ & $3.129530 \mathrm{E}-02$ & $1.074630 \mathrm{E}-01$ & $3.032230 E-01$ & $1.288730 \mathrm{E}+\odot \odot$ & 1. $044220 E+01$ \\
\hline $2.75477 \odot E-\odot 4$ & $1.165225 \mathrm{E}-03$ & 8. 999964E-๑4 & $2.034102 \mathrm{E}-03$ & $4.624642 \mathrm{E}-04$ & $6.164186 \mathrm{E}-05$ \\
\hline 92235 & $2.438879 \mathrm{E}+\odot \odot$ & & & & \\
\hline 1. 249056E- -2 & $3.182406 \mathrm{E}-02$ & 1. 093753E- 01 & $3.169898 \mathrm{E}-01$ & $1.353983 \mathrm{E}+\odot \odot$ & $8.636377 \mathrm{E}+00$ \\
\hline $2.183402 \mathrm{E}-04$ & $1.136937 \mathrm{E}-03$ & $1.102438 \mathrm{E}-03$ & $3.142815 E-03$ & $9.126013 \mathrm{E}-\odot 4$ & $3.225451 E-\odot 4$ \\
\hline 92236 & $2.585205 \mathrm{E}+0 \odot$ & & & & \\
\hline 1. 248999E- 02 & $3.068560 \mathrm{E}-02$ & 1. $091980 \mathrm{E}-01$ & $3.224705 \mathrm{E}-01$ & $1.378583 \mathrm{E}+\odot \odot$ & 1. $021014 \mathrm{E}+01$ \\
\hline $2.294104 \mathrm{E}-04$ & $1.726554 \mathrm{E}-03$ & 1. 473179E-๑3 & $3.893188 \mathrm{E}-03$ & $1.382420 \mathrm{E}-03$ & $2.693897 \mathrm{E}-04$ \\
\hline 92237 & $2.556079 \mathrm{E}+\odot \odot$ & & & & \\
\hline 1. 249577E-02 & $3.037978 \mathrm{E}-02$ & 1. 068975E-01 & $3.240317 \mathrm{E}-01$ & $1.334253 \mathrm{E}+\odot \odot$ & $9.544175 \mathrm{E}+0 \odot$ \\
\hline $2.081865 E-04$ & $2.419830 \mathrm{E}-03$ & 2. $019126 \mathrm{E}-\odot 3$ & $6.103966 \mathrm{E}-03$ & $2.421639 \mathrm{E}-03$ & $5.043196 E-04$ \\
\hline 92238 & $2.788314 \mathrm{E}+\odot \odot$ & & & & \\
\hline 1. 249423E-02 & 3. $025520 \mathrm{E}-02$ & 1. 159376E-01 & $3.414764 \mathrm{E}-01$ & $1.318630 \mathrm{E}+\odot \odot$ & $9.979027 \mathrm{E}+0 \odot$ \\
\hline $1.631869 \mathrm{E}-04$ & 1. 811873E- 03 & 2. 016816E-03 & $7.130047 \mathrm{E}-03$ & $3.684767 \mathrm{E}-\odot 3$ & $9.734561 E-04$ \\
\hline 93237 & $2.943373 \mathrm{E}+\odot \odot$ & & & & \\
\hline 1. $248610 \mathrm{E}-02$ & $3.077587 \mathrm{E}-02$ & 1. $065464 \mathrm{E}-01$ & $3.139911 \mathrm{E}-01$ & $1.334211 \mathrm{E}+0 \odot$ & 1. $050869 \mathrm{E}+01$ \\
\hline $1.169523 \mathrm{E}-04$ & $7.177156 \mathrm{E}-\odot 4$ & $6.106869 \mathrm{E}-04$ & 1.677948E- - & 4. 688961E-๑4 & $8.045746 E-05$ \\
\hline 94238 & $2.992942 \mathrm{E}+0 \odot$ & & & & \\
\hline $1.332500 \mathrm{E}-02$ & $3.117100 \mathrm{E}-02$ & $1.161500 \mathrm{E}-01$ & $2.888400 \mathrm{E}-01$ & $8.561400 \mathrm{E}-01$ & $2.713800 \mathrm{E}+00$ \\
\hline $5.261947 \mathrm{E}-05$ & $3.337788 \mathrm{E}-04$ & $2.202819 E-04$ & 4. $974348 E-04$ & $2.221220 E-\odot 4$ & $7.038188 E-05$ \\
\hline 94239 & $2.867711 \mathrm{E}+\Theta \odot$ & & & & \\
\hline $1.248110 \mathrm{E}-02$ & $2.994667 \mathrm{E}-02$ & 1. $071553 \mathrm{E}-01$ & $3.176193 \mathrm{E}-01$ & $1.352380 \mathrm{E}+\odot \odot$ & 1. $069116 \mathrm{E}+01$ \\
\hline $7.441243 E-05$ & $6.029267 E-04$ & $4.208394 \mathrm{E}-04$ & $8.788662 E-04$ & $2.338068 \mathrm{E}-04$ & $3.832867 E-05$ \\
\hline 94240 & $3.081800 \mathrm{E}+0 \odot$ & & & & \\
\hline $1.332900 \mathrm{E}-02$ & $3.051000 \mathrm{E}-02$ & 1. 151600E- 01 & $2.974000 \mathrm{E}-01$ & $8.476600 \mathrm{E}-\odot 1$ & $2.879600 \mathrm{E}+\odot \odot$ \\
\hline $9.336029 \mathrm{E}-05$ & $7.385726 \mathrm{E}-04$ & $4.4 \odot 4519 \mathrm{E}-\odot 4$ & $9.638943 E-04$ & $5.243305 \mathrm{E}-\odot 4$ & 1.597601E-๑4 \\
\hline 94241 & $2.946479 \mathrm{E}+\odot \odot$ & & & & \\
\hline 1. 359900E- 02 & $2.996600 \mathrm{E}-02$ & $1.167300 \mathrm{E}-01$ & 3. $069100 \mathrm{E}-01$ & $8.701000 \mathrm{E}-01$ & $3 . \odot \odot 280 \odot E+\odot \odot$ \\
\hline $9.923741 \mathrm{E}-05$ & $1.233243 E-03$ & $7.840723 E-04$ & 1. $920227 \mathrm{E}-03$ & 1. $086294 \mathrm{E}-03$ & $3.750161 \mathrm{E}-04$ \\
\hline 94242 & $3.146448 \mathrm{E}+0 \odot$ & & & & \\
\hline $1.360300 \mathrm{E}-02$ & $3.023800 \mathrm{E}-02$ & $1.15430 \odot \mathrm{E}-\odot 1$ & 3. $041900 \mathrm{E}-01$ & $8.27210 \odot \mathrm{E}-\odot 1$ & $3.137200 \mathrm{E}+\odot \odot$ \\
\hline 1. 229591E-๑4 & 1. 449077E- - 3 & $7.864419 E-\odot 4$ & 2. $042159 E-03$ & $1.412084 \mathrm{E}-03$ & $4.483058 \mathrm{E}-04$ \\
\hline 95241 & $3.236158 \mathrm{E}+0 \odot$ & & & & \\
\hline 1. 333800E- 02 & 3. $079800 \mathrm{E}-02$ & 1. 13050०E-01 & $2.867700 \mathrm{E}-01$ & $8.653600 \mathrm{E}-01$ & $2.643000 \mathrm{E}+00$ \\
\hline $4.687282 E-05$ & $3.351367 \mathrm{E}-04$ & $2.062589 E-04$ & $4.438472 E-04$ & $2.274633 \mathrm{E}-\odot 4$ & $5.988720 E-05$ \\
\hline 95243 & $3.564782 \mathrm{E}+00$ & & & & \\
\hline 1. 349900E- 02 & $2.975900 \mathrm{E}-\odot 2$ & $1.137700 \mathrm{E}-01$ & $2.985900 \mathrm{E}-01$ & $8.820200 \mathrm{E}-01$ & $2.811100 \mathrm{E}+0 \odot$ \\
\hline $5.213651 \mathrm{E}-05$ & $6.568510 \mathrm{E}-\odot 4$ & $3.428585 \mathrm{E}-\odot 4$ & $7.020686 \mathrm{E}-04$ & $3.694160 \mathrm{E}-04$ & 1. $071179 \mathrm{E}-04$ \\
\hline 96242 & $3.753388 \mathrm{E}+00$ & & & & \\
\hline 1. 295600E-02 & $3.124500 \mathrm{E}-02$ & 1. 129100E-01 & $2.783400 \mathrm{E}-01$ & 8.710400E-01 & $2.196900 \mathrm{E}+00$ \\
\hline $2.760101 \mathrm{E}-05$ & 1. $027275 \mathrm{E}-\odot 4$ & $5.117072 \mathrm{E}-05$ & 1. $024804 \mathrm{E}-\odot 4$ & $6.385763 \mathrm{E}-05$ & 1. $359061 \mathrm{E}-05$ \\
\hline 96245 & $3.597515 \mathrm{E}+\odot \odot$ & & & & \\
\hline 1. 340०००E- -2 & 3. $070000 \mathrm{E}-02$ & 1. 1300००E- $\odot 1$ & $3.001000 \mathrm{E}-01$ & 8. 3400९९E- -1 & $2.768600 \mathrm{E}+0 \odot$ \\
\hline $3.942668 \mathrm{E}-05$ & $3.180151 \mathrm{E}-04$ & $2.974124 \mathrm{E}-04$ & $6.592391 E-04$ & $3.654310 \mathrm{E}-\odot 4$ & $9.948328 E-05$ \\
\hline 98249 & $3.888388 \mathrm{E}+00$ & & & & \\
\hline $1.351700 \mathrm{E}-02$ & $2.945000 \mathrm{E}-02$ & 1. 053200E-01 & $2.929800 \mathrm{E}-01$ & $8.474900 \mathrm{E}-01$ & $2.469800 \mathrm{E}+00$ \\
\hline 1. $709062 \mathrm{E}-05$ & $2.721421 \mathrm{E}-04$ & $9.365231 E-05$ & 1. $8 \odot 4290 \mathrm{E}-\odot 4$ & $1.120952 \mathrm{E}-\odot 4$ & $1.896568 \mathrm{E}-05$ \\
\hline 98251 & $4.140665 \mathrm{E}+0 \odot$ & & & & \\
\hline 1.569700E- 02 & $2.883000 \mathrm{E}-02$ & 1. $076900 \mathrm{E}-01$ & $3.245800 \mathrm{E}-01$ & 8. 837100E - 01 & $2.631400 \mathrm{E}+0 \odot$ \\
\hline $9.953225 E-06$ & $6.522987 \mathrm{E}-\odot 4$ & $3.156976 \mathrm{E}-\odot 4$ & 4. 898075E-๑4 & $3.069656 \mathrm{E}-04$ & 4.397731E-05 \\
\hline
\end{tabular}

Figure 5.2.2 Transient data from ENDF/B-7.0

\subsection{Effective recoverable energy per fission}

The energy release per fission is required for burnup calculations, and is usually defined without the kinetic energy of the incident neutrons and the energy carried away by neutrinos. However, the energy release per fission includes the contributions from the kinetic energy of incident neutrons and from the decay of the capture products: 


$$
W_{\text {fiss }}^{i}=E_{r}^{i}+Q_{c}^{i}+W_{n}^{i},
$$

where $W_{\text {fiss }}^{i}$ is the effective energy release in fission, $E_{r}^{i}$ is the energy release in fission excluding the energy carried away by neutrinos and the kinetic energy of the incident neutrons, $Q^{i}{ }_{c}$ is the contribution from the decay of the capture products, and $W_{n}^{i}$ is the kinetic energy of the incident neutrons. Index $i$ refers to a fissionable nuclide in all cases.

$E_{r}^{i}$ values are extracted from the $\mathrm{MF}=1 / \mathrm{MT}=458$ section of an evaluated nuclear data file, while the kinetic energy of the incident neutron is obtained by approximately averaging the energy with the fission reaction rate. The averaging process involved the multi-group approximation and the cross sections obtained from the MCNP calculations based on ENDF/B cross section data:

$$
W_{n}^{i}=\frac{\sum_{g} \bar{E}_{g} \sigma_{f, g}^{i} \phi_{g}}{\sum_{g} \sigma_{f, g}^{i} \phi_{g}},
$$

where $\sigma_{f, g}^{i}$ is the microscopic fission cross section of the fissionable nuclide $i$ in the energy group $g, \phi_{g}$ is the neutron spectrum, and $E_{g}$ is the average group energy. A typical PWR fuel pin was used in this calculation.

The average group energy was a simple mean of the group boundary values, except for the highest energy groups for which more accurate values were adopted to give the average energy in a Maxwellian fission spectrum at a temperature corresponding to $1.4 \mathrm{MeV}$ :

$$
E_{g}=\frac{\int_{g} E \sqrt{E} \cdot \exp (-E / T) d E}{\int_{g} \sqrt{E} \cdot \exp (-E / T) d E} .
$$

The additional energy released due to gamma activation is calculated from

$$
Q_{c}=(\bar{v}-1) Q,
$$

where $(\bar{v}-1)$ represents the average number of neutrons that are captured, and $Q=6.1 \mathrm{MeV}$ [Jam99].

\subsection{Effective background cross sections}

While the AMPX MG library includes resonance data for all nuclides and energy groups, the MPACT library includes resonance data for the specified nuclides and energy groups. For example, 49 nuclides include subgroup data and resonance integral tables and 191 nuclides out of 295 include IR parameters only for groups 10 26. If infinitely dilute cross sections are provided in the MPACT MG library, the MPACT results would underestimate the multiplication 
factors at very high burnup points due to higher capture cross sections. Therefore, pre-selfshielded cross sections need to be included in the MPACT MG library.

At low burnup points heavy and fission product nuclides produced by decay and neutron reactions do not significantly contribute on the multiplication factor due to tiny amount of atomic number densities. It is reasonable to determine high burnup point at which background cross sections are estimated to be used in obtaining 1D self-shielded cross sections. This work can be done by the MPACT single pin depletion calculation to obtain atomic number density at high burnup point (50 MWD/kgU) and then effective microscopic background cross sections can be obtained from the MPACT subgroup or ESSM calculations. 


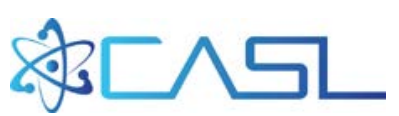

\section{GENERATION OF THE MPACT MG LIBRARY}

\section{$\underline{6.1 \text { Generation of the initial AMPX MG library }}$}

The AMPX MG library can be generated by using the program EXSITE by which all the AMPX module input files for all nuclides can be automatically prepared through expanding a template shown in Figure 6.1.1. Figure 6.1.2 provides the AMPX procedure to generate the AMPX MG library where the left side of flow chart is for the conventional procedure and the right side for new procedure to improve the Bondarenko resonance data. The following data are required in generating the AMPX MG library.

- $\quad$ ENDF/B-VII neutron cross section data

- ENDF/B-VII listing file

- ENDF/B-VII Doppler broadening data files

- ENDF/B-VII probability table files

- Pointwise weighting function

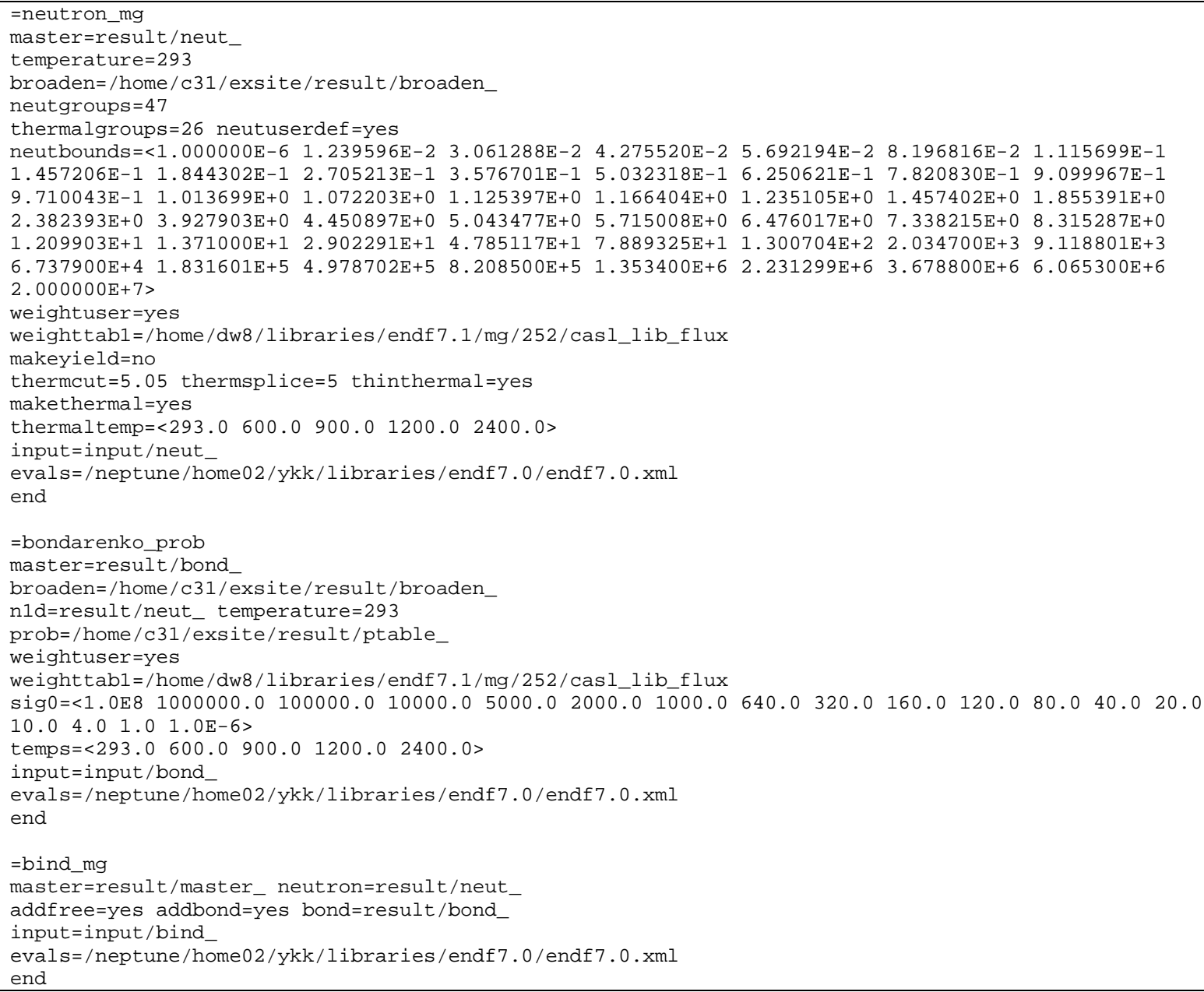

Figure 6.1.1 Sample template to generate the AMPX input files 




Figure 6.1.2 Flow chart of the AMPX procedure

After completing the AMPX calculations for all nuclides, individual MG data files can be merged into an AMPX MG library (I) by the AMPX AJAX module.

\section{$\underline{6.2 \text { Intermediate resonance parameters and Homogeneous F-factors }}$}

The intermediate resonance (IR) parameters are generated for all nuclides by using the AMPXLAMBDA module with a procedure introduced in Section 3.3 in which the IR parameters are estimated only for the specified energy groups (for example, the 47-g library for groups 10 46) and unity is assigned to other energy groups. In addition homogeneous F-factors are generated for the same energy groups for the IR parameters by the AMPX-IRFFACTOR-hom module with a procedure described in Section 3.4 which are substituted for the original F-factors.

Figure 6.2.1 provides a template to be expanded to generate the LAMBDA and IRFFACTORhom input files for all nuclides. An expanded sample input for ${ }^{235} \mathrm{U}$ is shown in Appendix A.1. Figure 6.2.2 provides a flow chart of the resonance data generation procedure in which pointwise slowing down calculations are performed by CENTRM. The following data are included in the AMPX MG master library.

- Intermediate resonance parameters : all nuclides, all energy groups

- Homogeneous F-factors : Nuclides Atomic number $\geq 40$ (Zr) 


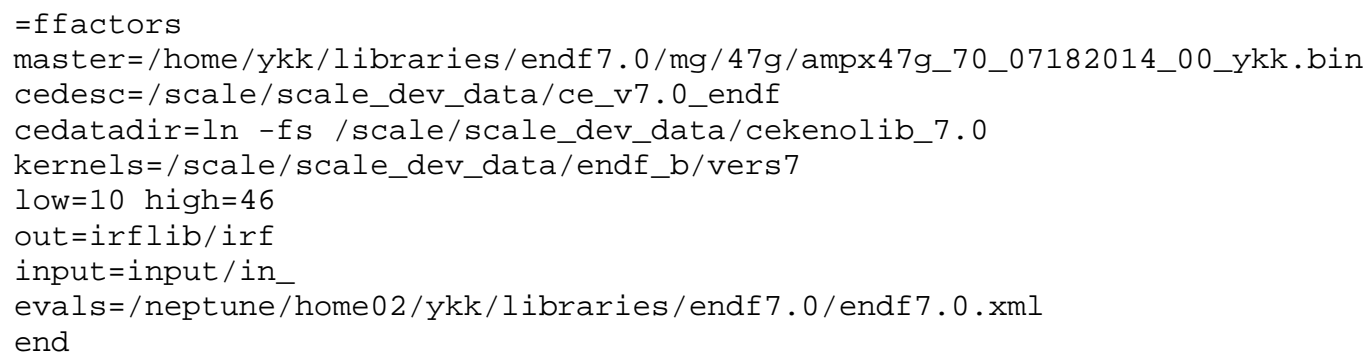

Figure 6.2.1 Sample template to generate the IR parameters and homogeneous F-factors

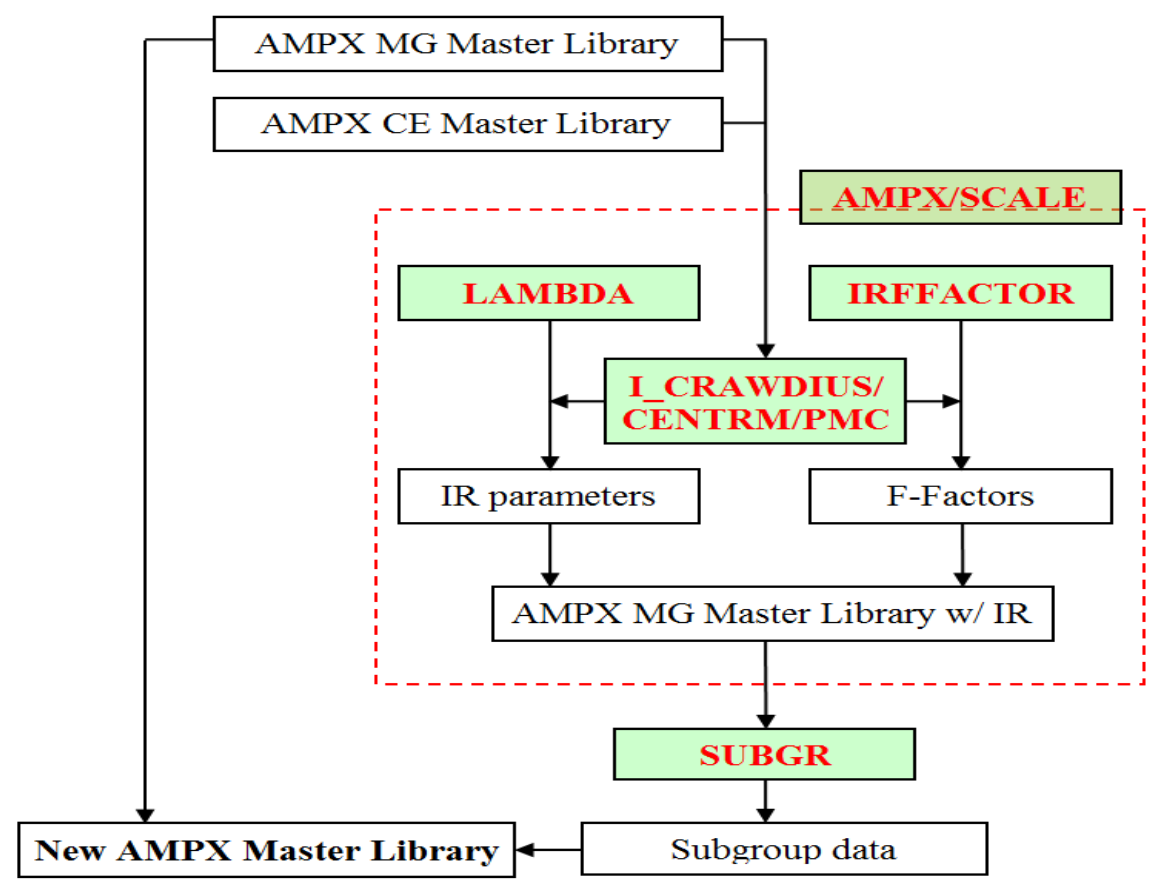

Figure 6.2.1 Flow chart of the resonance data generation procedure

\section{$\underline{6.3 \text { Heterogeneous F-factors }}$}

Only selected nuclides include heterogeneous F-factors which are very important resonance nuclides significantly impacting on the neutronics result. The following 20 nuclides have been selected to have heterogeneous F-factors. Option for level dependent background cross sections has been used for all nuclides except for Ag, In and Cd nuclides.

${ }^{107} \mathrm{Ag}, \quad{ }^{109} \mathrm{Ag}, \quad{ }^{113} \mathrm{Cd},{ }^{232} \mathrm{Th},{ }^{113} \mathrm{In}, \quad{ }^{235} \mathrm{U},{ }^{236} \mathrm{U},{ }^{238} \mathrm{U},{ }^{115} \mathrm{In}, \quad{ }^{239} \mathrm{Pu},{ }^{155} \mathrm{Pu},{ }^{240} \mathrm{Pu},{ }^{241} \mathrm{Pu},{ }^{242} \mathrm{Pu},{ }^{241} \mathrm{Am}$ 
The IRFFACTOR-het input file for ${ }^{238} \mathrm{U}$ to generate heterogeneous F-factors is shown in Figure 6.3.1 and a companion input file for various geometrical and compositional configurations is provided in Appendix B.1. Since typically Bondarenko approach is not able to consider resonance interference effect explicitly, heterogeneous F-factors can be generated in two different ways with or without considering explicit resonance interference. 'Single' and 'Multiple' indicate without and with resonance interference, respectively. Both models are applied only to 5 heavy nuclides including ${ }^{235} \mathrm{U},{ }^{238} \mathrm{U},{ }^{239} \mathrm{Pu},{ }^{240} \mathrm{Pu}$, and ${ }^{241} \mathrm{Pu}$.

The Heterogeneous IRFFACTOR results in two F-factor tables of which the first one can be incorporated by the AMPX-AJAX and the second one (subgrpdata) is for SUBGR to generate subgroup data. The first one (or the AMPX MG master library) also can be utilized in the subgroup data generation. However, in order to generate subgroup data by using eq. (4.2.7) to conserve cross sections, subgroup level dependent background cross sections should be added to the F-factor tables (self-shielded cross section table). The AMPX MG library does not include the subgroup level dependent background cross sections, but the 'subgrpdata' file includes them.

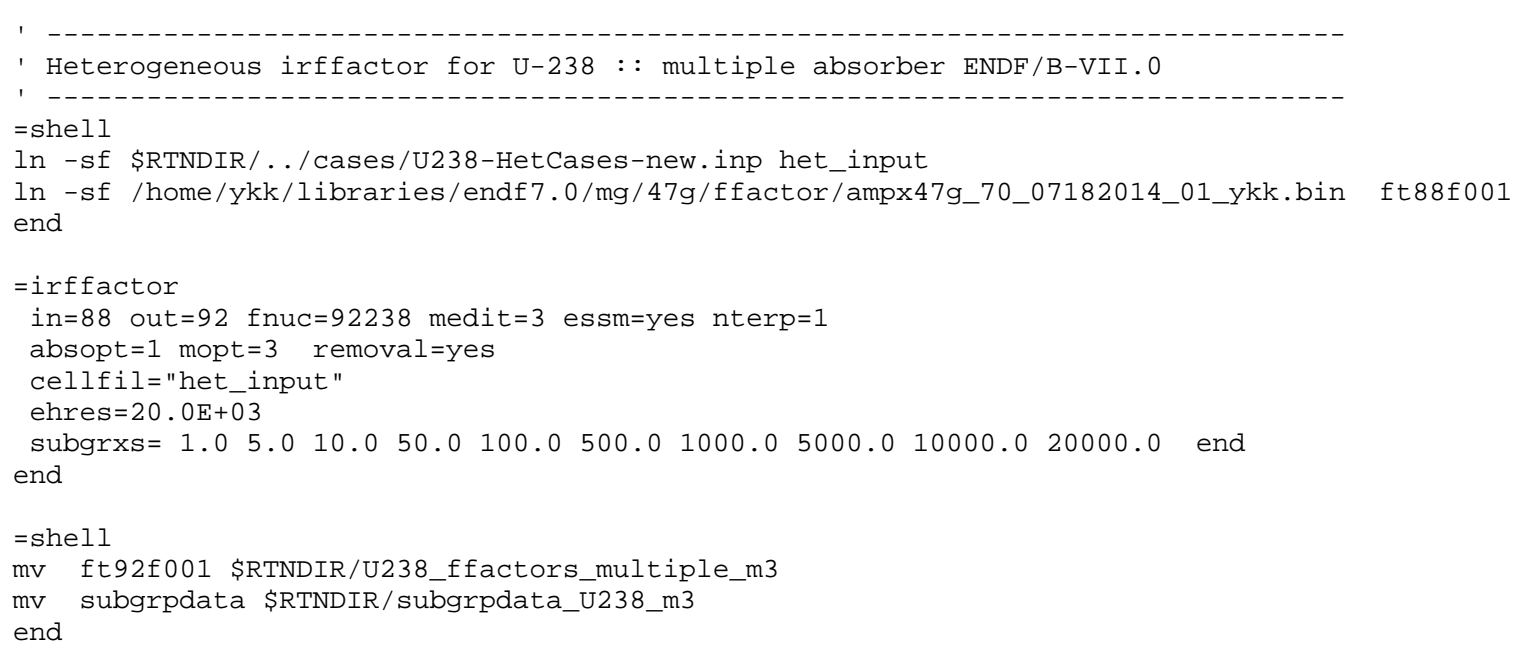

Figure 6.3.1 Sample input to generate the heterogeneous F-factors

\section{$\underline{6.4 \text { Generation of subgroup data }}$}

At first Bondarenko F-factors in the AMPX MG library should be converted into resonance integral tables to be used in the subgroup data generation. And then subgroup data are generated by using SUBGR. Subgroup data including weights and levels have been generated for important 49 resonance nuclides as shown in Table 6.4.1 and for all energy groups. Figures 6.4.1 and 6.4.2 provide the standard subgroup level and the SUBGR input files, respectively. 
Table 6.4.1 List of nuclides including subgroup data

\begin{tabular}{|c|c|c|c|c|c|c|c|c|c|c|c|}
\hline No & Nuclide & ID & Method & $\sigma_{a}$ & $v \sigma_{f}$ & No & Nuclide & ID & Method & $\sigma_{a}$ & $v \sigma_{f}$ \\
\hline 1 & ${ }^{91} \mathrm{Zr}$ & 40091 & RI & 0 & $\mathrm{x}$ & 26 & ${ }^{163} \mathrm{Dy}$ & 66163 & RI & 0 & $\mathrm{x}$ \\
\hline 2 & ${ }^{96} \mathrm{Zr}$ & 40096 & RI & 0 & $\mathrm{x}$ & 27 & ${ }^{164} \mathrm{Dy}$ & 66164 & RI & 0 & $\mathrm{x}$ \\
\hline 3 & 每5 $\mathrm{Mo}$ & 42095 & RI & 0 & $\mathrm{x}$ & 28 & ${ }^{166} \mathrm{Er}$ & 68166 & RI & 0 & $\mathrm{x}$ \\
\hline 4 & ${ }^{99} \mathrm{Tc}$ & 43099 & RI & 0 & $\mathrm{x}$ & 29 & ${ }^{167} \mathrm{Er}$ & 68167 & RI & 0 & $\mathrm{x}$ \\
\hline 5 & ${ }^{103} \mathrm{Rh}$ & 45103 & RI & 0 & $\mathrm{x}$ & 30 & ${ }^{176} \mathrm{Hf}$ & 72176 & RI & 0 & $\mathrm{x}$ \\
\hline 6 & ${ }^{108} \mathrm{Pd}$ & 46108 & RI & 0 & $\mathrm{x}$ & 31 & ${ }^{177} \mathrm{Hf}$ & 72177 & RI & 0 & $\mathrm{x}$ \\
\hline 7 & ${ }^{107} \mathrm{Ag}$ & 47107 & RI & 0 & $\mathrm{x}$ & 32 & ${ }^{178} \mathrm{Hf}$ & 72178 & RI & 0 & $\mathrm{x}$ \\
\hline 8 & ${ }^{109} \mathrm{Ag}$ & 47109 & RI & 0 & $\mathrm{x}$ & 33 & ${ }^{179} \mathrm{Hf}$ & 72179 & RI & 0 & $\mathrm{x}$ \\
\hline 9 & ${ }^{113} \mathrm{In}$ & 49113 & RI & 0 & $\mathrm{x}$ & 34 & ${ }^{180} \mathrm{Hf}$ & 72180 & RI & 0 & $\mathrm{x}$ \\
\hline 10 & ${ }^{115} \mathrm{In}$ & 49115 & RI & 0 & $\mathrm{x}$ & 35 & ${ }^{182} \mathrm{~W}$ & 74182 & RI & 0 & $\mathrm{x}$ \\
\hline 11 & ${ }^{131} \mathrm{Xe}$ & 54131 & RI & 0 & $\mathrm{x}$ & 36 & ${ }^{183} \mathrm{~W}$ & 74183 & RI & 0 & $\mathrm{x}$ \\
\hline 12 & ${ }^{133} \mathrm{Cs}$ & 55133 & RI & 0 & $\mathrm{x}$ & 37 & ${ }^{184} \mathrm{~W}$ & 74184 & RI & 0 & $\mathrm{x}$ \\
\hline 13 & ${ }^{152} \mathrm{Sm}$ & 62152 & RI & 0 & $\mathrm{x}$ & 38 & ${ }^{186} \mathrm{~W}$ & 74186 & RI & 0 & $\mathrm{x}$ \\
\hline 14 & ${ }^{151} \mathrm{Eu}$ & 63151 & RI & 0 & $\mathrm{x}$ & 39 & ${ }^{232} \mathrm{Th}$ & 90232 & $\mathrm{XS}$ & 0 & $\mathrm{x}$ \\
\hline 15 & ${ }^{152} \mathrm{Eu}$ & 63152 & RI & 0 & $\mathrm{x}$ & 40 & ${ }^{233} \mathrm{U}$ & 92233 & XS & 0 & 0 \\
\hline 16 & ${ }^{153} \mathrm{Eu}$ & 63153 & RI & 0 & $\mathrm{x}$ & 41 & ${ }^{235} \mathrm{U}$ & 92235 & $\mathrm{XS}$ & 0 & 0 \\
\hline 17 & ${ }^{154} \mathrm{Eu}$ & 63154 & $\mathrm{RI}$ & 0 & $\mathrm{x}$ & 42 & ${ }^{236} \mathrm{U}$ & 92236 & $\mathrm{XS}$ & 0 & 0 \\
\hline 18 & ${ }^{155} \mathrm{Eu}$ & 63155 & RI & 0 & $\mathrm{x}$ & 43 & ${ }^{238} \mathrm{U}$ & 92238 & $\mathrm{XS}$ & 0 & $\mathrm{x}$ \\
\hline 19 & ${ }^{155} \mathrm{Gd}$ & 64155 & XS & 0 & $\mathrm{x}$ & 44 & ${ }^{238} \mathrm{Pu}$ & 94238 & XS & 0 & 0 \\
\hline 20 & ${ }^{156} \mathrm{Gd}$ & 64156 & XS & 0 & $\mathrm{x}$ & 45 & ${ }^{239} \mathrm{Pu}$ & 94239 & XS & 0 & 0 \\
\hline 21 & ${ }^{157} \mathrm{Gd}$ & 64157 & XS & 0 & $\mathrm{x}$ & 46 & ${ }^{240} \mathrm{Pu}$ & 94240 & XS & 0 & 0 \\
\hline 22 & ${ }^{158} \mathrm{Gd}$ & 64158 & $\mathrm{XS}$ & 0 & $\mathrm{x}$ & 47 & ${ }^{241} \mathrm{Pu}$ & 94241 & $\mathrm{XS}$ & 0 & 0 \\
\hline 23 & ${ }^{160} \mathrm{Dy}$ & 66160 & RI & 0 & $\mathrm{x}$ & 48 & ${ }^{242} \mathrm{Pu}$ & 94242 & $\mathrm{XS}$ & 0 & 0 \\
\hline 24 & ${ }^{161}$ Dy & 66161 & RI & 0 & $\mathrm{x}$ & 49 & ${ }^{241} \mathrm{Am}$ & 95241 & XS & 0 & 0 \\
\hline 25 & ${ }^{162} \mathrm{Dy}$ & 66162 & RI & 0 & $\mathrm{x}$ & & & & & & \\
\hline
\end{tabular}

RI : Resonance Integral conservation

XS: Cross section conservation

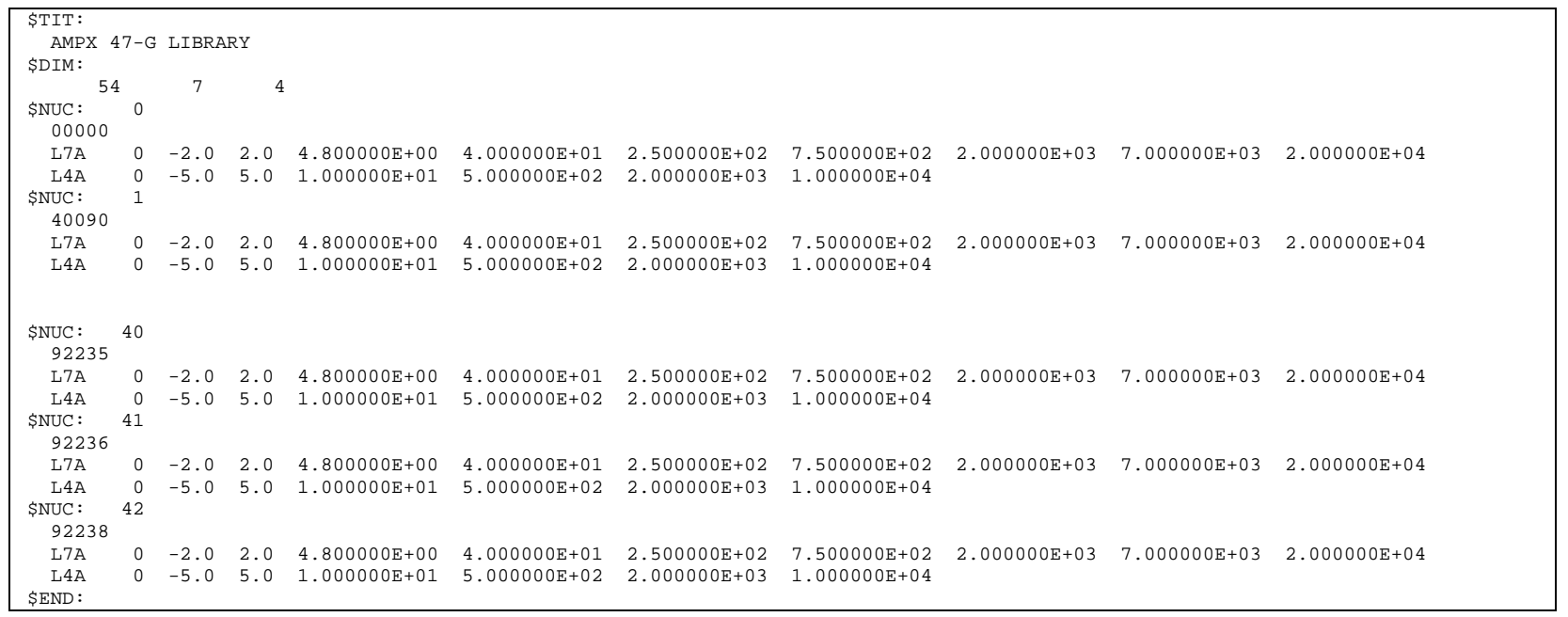

Figure 6.4.1 Sample of the initial subgroup level 




Figure 6.4.2 Sample of the SUBGR input

\section{$\underline{6.5 \text { Generation of the MPACT MG library }}$}

Appendices A and B provide the detailed data information for the MPACT MG library and the DECLIB input to generate the ENDF/B-7.1 based V4.0 MPACT 47-group library. The following data files are required for DECLIB to generate the MPACT MG library.

- $\quad$ The AMPX 47-g library

- ENDF/B files : Neutron data, decay constants, fission product yields

- Subgroup data and resonance integral table (49 nuclides, groups 10-26)

- Transport correction factors $\left({ }^{1} \mathrm{H}\right)$

- $\quad$ Pre-determined background cross sections (105 nuclides) 
- Transient data (21 nuclides)

- Subgroup data with epithermal upscattering $\left({ }^{238} \mathrm{U}\right)$

Figure 6.5.1 provides a flow diagram of DECLIB to generate the MPACT MG library of which data structure is shown in Appendix C.

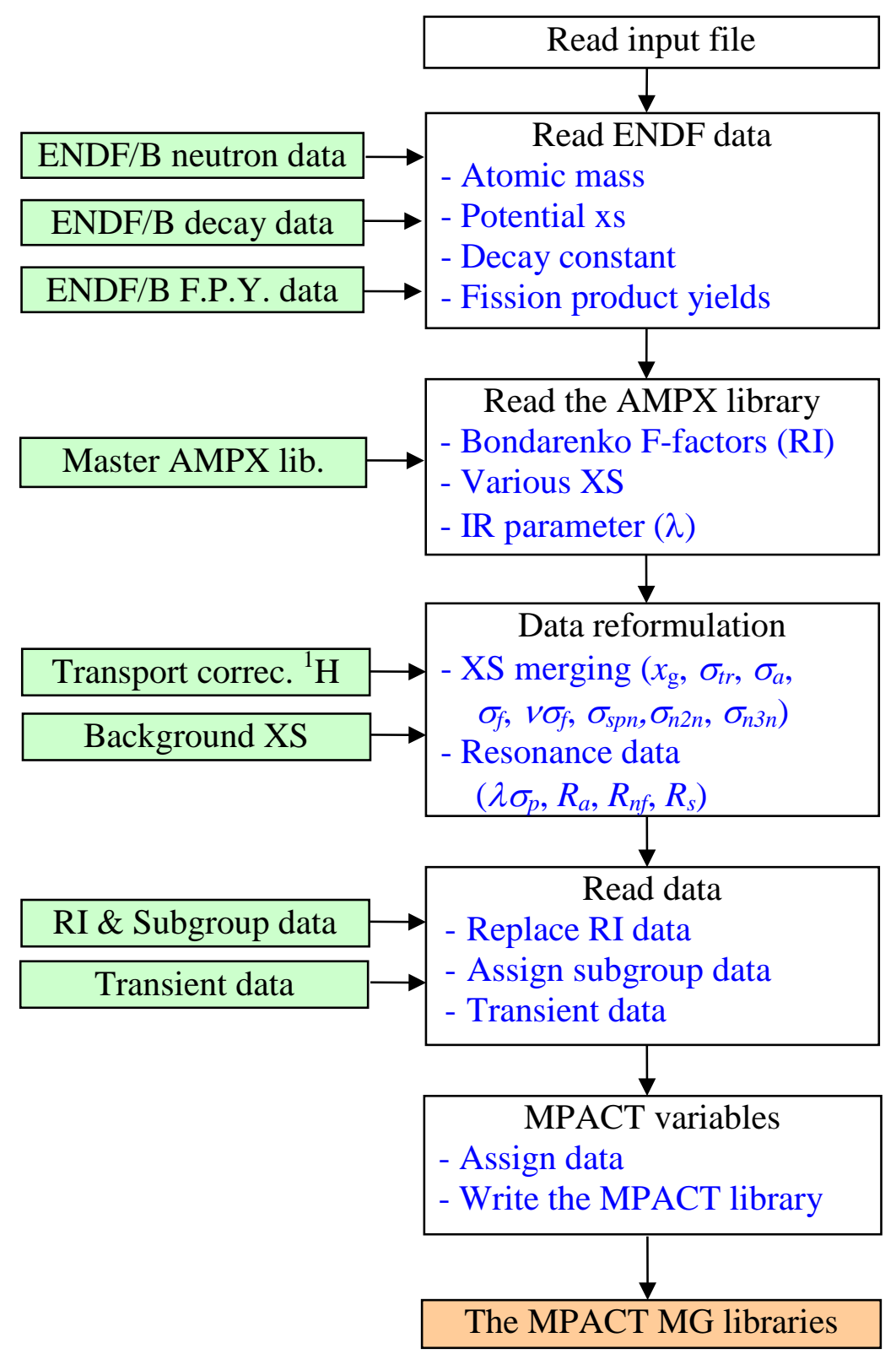

Figure 6.5.1 DECLIB calculation flow 
Multigroup cross sections are directly edited from the AMPX master library. MPACT solves the time-independent Boltzmann equation as follows:

$$
\hat{\Omega} \cdot \nabla \psi_{g}+\Sigma_{t r, g}(\vec{r}) \psi_{g}(\vec{r}, \hat{\Omega})=\sum_{g^{\prime}} \Sigma_{s g^{\prime} g} \phi_{g^{\prime}}+\frac{1}{k_{e f f}} \chi_{g} \sum_{g^{\prime}} v \Sigma_{f g^{\prime}} \phi_{g^{\prime}},
$$

where

$$
\begin{array}{ll}
\hat{\Omega} & : \text { neutron direction } \\
\vec{r} & : \text { space coordinate } \\
\mathrm{g} & : \text { neutron energy group } \\
\psi_{g} & : \text { angular flux } \\
\phi_{g} & : \text { scalar flux } \\
\Sigma_{t r, g} & : \text { macroscopic transport cross section } \\
\Sigma_{s, g^{\prime} g} & : \text { transport corrected scattering matrix } \\
v & : \text { the number of neutrons released from a fission } \\
\Sigma_{f, g^{\prime}} & : \text { fission cross section } \\
\chi_{g} & : \text { fission spectrum } \\
k_{e f f} & : \text { fffective multiplication factor. }
\end{array}
$$

The required data for the transport calculation are transport cross section, fission cross section, the number of neutrons released from a fission, and fission spectrum for each nuclide. Since the absorption and fission cross sections are modified through the resonance treatment and are needed for the depletion calculation, those cross sections should be included. And (n,2n) and $(n, 3 n)$ cross sections are required for the depletion cross sections. High order $\left(\mathrm{P}_{1} \sim \mathrm{P}_{3}\right)$ scattering matrices are to be included for the future use. Therefore, the multigroup data required in the library are as follows:
a. Total cross section $\left(\sigma_{t}\right)$
b. Absorption cross section $\left(\sigma_{a}=\sigma_{c^{+}} \sigma_{f}\right)$
c. Fission cross section $\left(\sigma_{f}\right)$
d. Neutrons released from a fission $(v)$
e. Scattering cross sections $\left(\sigma_{s 0 \sim 3}\right)$
f. $(n, 2 n)$ cross section $\left(\sigma_{n, 2 n}\right)$
g. $(n, 3 n)$ cross section $\left(\sigma_{n, 3 n}\right)$
h. $\mathrm{P}_{0 \sim 3}$ scattering matrix $\left(\sigma_{\text {sngg }}\right)$
i. Fission spectrum $\left(\chi_{g}\right)$

Since the AMPX master library includes cross section data for various reactions, it is required to merge cross sections to generate the above underlined data. Table 6.5.1 provides which MT numbers are to be merged for the required data.

$(n, 2 n)$ and $(n, 3 n)$ cross sections are not treated explicitly, but included in the absorption cross section and scattering matrix as follows: 


$$
\sigma_{a, g}^{c}=\sigma_{c, g}+\sigma_{f, g}-\sigma_{n 2 n, g}-2 \sigma_{n 3 n, g}
$$

and

$$
\sigma_{s, g g^{\prime}}^{c}=\sigma_{s, g g^{\prime}}+2 \sigma_{n 2 n, g g^{\prime}}+3 \sigma_{n 3 n, g g^{\prime}}
$$

The effective fission spectrum needs to be re-estimated by using MT=18, 452, 455, 1055 and 1099 by using the following formula.

$$
\chi_{g}=\frac{\chi_{g}^{\text {prompt }} \sum_{g^{\prime}} v_{g^{\prime}}^{\text {prompt }} \sigma_{g^{\prime}} \phi_{g^{\prime}}+\chi_{g}^{\text {delayed }} \sum_{g^{\prime}} v_{g^{\prime}}^{\text {delayed }} \sigma_{g^{\prime}} \phi_{g^{\prime}}}{\sum_{g^{\prime}} v_{g^{\prime}}^{\text {prompt }} \sigma_{g^{\prime}} \phi_{g^{\prime}}+\sum_{g^{\prime}} v_{g^{\prime}}^{\text {delayed }} \sigma_{g^{\prime}} \phi_{g^{\prime}}} .
$$

Table 6.5.1 MT numbers to be merged

\begin{tabular}{|c|c|c|c|}
\hline \multirow{2}{*}{ Cross section } & \multicolumn{3}{|c|}{ MT numbers in the AMPX master library } \\
\cline { 2 - 4 } & F-factors & 1 -D cross section & 2-D cross section \\
\hline$\sigma_{t}$ & 1 & - & - \\
\hline$\sigma_{c}$ & 102 & $103 \sim 117$ & - \\
\hline$\sigma_{f}$ & 18 & - & - \\
\hline$v$ & - & 452 & - \\
\hline$\sigma_{s 0}$ & 2 & 4 & 11,16 \\
\hline$\sigma_{n, 2 n}$ & - & 11,16 & 17 \\
\hline$\sigma_{n, 3 n}$ & - & 17 & $2,50 \sim 91,1007,1008$ \\
\hline$\sigma_{\text {sngg }}$ & - & - & - \\
\hline$\chi_{g}{ }^{*}$ & - & $18,452,455,1055,1056,1099$ & - \\
\hline$\lambda$ & - & 2000 & - \\
\hline$\sigma_{s, g}^{\text {within }}$ & 2022 & - & \\
\hline
\end{tabular}

*Since MT=1018 is just for prompt neutron, $\chi_{g}$ is internally recalculated. 


\section{CHALLENGING ISSUES}

\section{$\underline{7.1 \text { Drawbacks of the MPACT MG library }}$}

The current MPACT 47-group library includes the following drawbacks.

- 49 nuclides include full resonance data such as subgroup data, RI table and IR parameters

- Groups 10 through 26 include resonance data.

- Pre-self-shielded cross sections should be utilized for non-resonance nuclides and nonresonance energy groups.

- Temperature independent elastic scattering matrices are available. (This is a drawback of the AMPX MG library.)

- Although the ENDF/B-7.0 AMPX MG library includes 419 nuclides, the MPACT MG library include 295 nuclides and in addition some of nuclides are duplicated for activation and fission product nuclides.

- Many of fission product nuclides include only absorption cross sections.

- There are many duplicated nuclides at activation and fission product nuclides.

- High order scattering matrices $\left(\geq \mathrm{P}_{2}\right)$ are available only for important nuclides.

\section{$\underline{7.2 \text { Resonance interference }}$}

Self-shielded cross section table can be generated by considering resonance interference effect between resonance nuclides such as ${ }^{238} \mathrm{U}$ and ${ }^{235} \mathrm{U}$ for the CENTRM pointwise slowing down calculation. This method may be able to provide better agreement for the specific cases in MG 1D self-shielded cross sections or reaction rates between reference solution and the MPACT result. However, resonance interferences are doubly considered due to the Bondarenko iteration of subgroup method for resonance interference, and this procedure would introduce significant ${ }^{235} \mathrm{U}$ enrichment bias resulting in radial power tilt at the whole core calculation.

Resonance interference could not be considered explicitly in the conventional Bondarenko resonance self-shielding methods such as subgroup method, ESSM and Dancoff based methods when using coarse energy group structure. There can be several solutions as follows:
a. Pre-calculated resonance interference factor tables [Kim11]
b. ESSM+0D slowing down [Kim12]
c. ESSM-X [Liu15]

However, these methods are still under development and include various pending technical issues associated with computing time and accuracy. These will be discussed later.

\section{$\underline{7.3 \text { Resonance self-shielding effect on scattering matrix }}$}

In deterministic transport calculation, the accuracy is determined by quality of both $1 \mathrm{D}$ and $2 \mathrm{D}$ cross section data. Recently new F-factor table for removal correction has been included to 


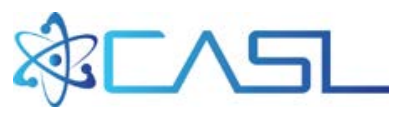

adjust scattering matrix in the AMPX MG master library. It is noted that this approach is very successful in resolving temperature bias problem due to temperature independent scattering matrix. At first a new functionality to utilize the F-factor table for removal correction needs to be incorporated into MPACT. However, since this approach is based on Bondarenko method, there should be some bias in estimating self-shielding effect in scattering matrix. New resonance selfshielding method is based on quasi 1D slowing down calculation. Therefore, removal correction factor for 2D data can be explicitly estimated simultaneously with an estimation of 1D data. These removal correction factors can be applied to scattering matrix.

\subsection{Intra pin self-shielding effect in ESSM}

When estimating resonance self-shielded cross section by ESSM, absorption cross sections $\left(\Sigma_{i, a, g}\right)$ at fuel and cladding are iteratively determined by solving the fixed source transport eq. (7.4.1).

$$
\hat{\Omega} \cdot \nabla \psi_{g}+\sum_{i}\left(\Sigma_{i, a, g}+\lambda_{i, g} \Sigma_{i, p}\right) \psi_{g}(\hat{\Omega})=\sum_{i} \lambda_{i, g} \Sigma_{i, p}
$$

Typical PWR and BWR fuel pins include $\mathrm{UO}_{2}$ fuel and zirconium alloy fuel cladding in which ${ }^{235} \mathrm{U},{ }^{238} \mathrm{U},{ }^{91} \mathrm{Zr}$ and ${ }^{95} \mathrm{Zr}$ are important resonant nuclides requiring explicit resonance treatment. Since $\mathrm{Zr}$ nuclides are located at different zone from fuel, there is no direct resonance interference effect with fuel nuclides.

When generating F-factor tables by using homogeneous or heterogeneous models by the SCALE and AMPX procedures, only one resonant nuclide is considered to have absorption cross section and other background nuclides include only potential or scattering cross sections in slowing down and ESSM calculations. Multigroup cross sections are obtained from the slowing down calculations and the corresponding background cross sections are obtained by solving eq. (7.4.1) and then by using eq. (7.4.2).

$$
\Sigma_{b, g}=\frac{\Sigma_{a, g} \phi_{g}}{1-\phi_{g}} \rightarrow \sigma_{b, g}^{i}=\frac{\Sigma_{b, g}}{N_{i}}
$$

When performing multigroup ESSM calculation, absorption cross sections at fuel and cladding are estimated simultaneously. Since the scalar flux $\left(\phi_{g}\right)$ at fuel is influenced by the cladding absorption cross section and vice versa, self-shielded cross sections at fuel and cladding introduce some bias because of inconsistency between how to generate F-factor table and how to use it.

In order to avoid this problem, the AMPX 56-group master library includes two sets of cross section data for ${ }^{91} \mathrm{Zr}$ and ${ }^{96} \mathrm{Zr}$. The first set of F-factor table was prepared by using a conventional procedure, and the second set of F-factor table was generated by using a model shown in Figure 
7.4.1 (a). The second set of F-factor tables cannot be utilized in other composition except for fuel cladding. While the impact on $\mathrm{Zr}$ nuclides of cladding by fuel is significant, the impact on $\mathrm{U}$ nuclides of fuel by cladding is not. In order to have the highest quality of self-shielded cross sections and a simplified procedure, this impact should be considered for both cladding and fuel with a utilization of only one set of F-factor table.

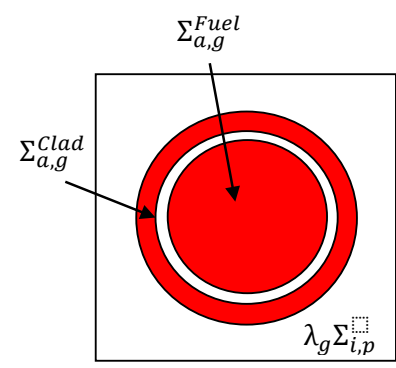

(a)



(b)

Figure 7.4.1 Counter influence between fuel and cladding

In order to solve this problem category concept in the subgroup method can be utilized. Or if fuel and cladding regions are separated from each other which is somewhat similar with category concept, this problem can be resolved which may be called a geometrical category.

Another idea is to include a special option to generate a table of macroscopic cross sections as a function of temperature for which resonance self-shielding effect, if necessary, can be considered by using a simple model such as single pin and homogeneous mixture. This option can be applied to cladding, detector and moderator. Similar approach can be applied to the subgroup method.

\section{ACKNOWLEDGEMENT}

The author would like to acknowledge Kevin T. Clarno who is the CASL PHI lead for his efforts to review this document in detail and provide valuable comments. 


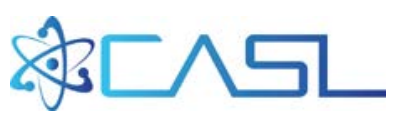

\section{REFERENCE}

[Ald03] D. L. Aldama and et. al., “WIMS-D Library Update,” IAEA (2003).

[Bec10] B. Becker, "On the influence of the Resonance Scattering Treatment in Monte Carlo Codes on High Temperature Reactor Characteristics," Ph. D. Thesis, University Stuttgart, June, 2010 (2010).

[Dud76] James J. Duderstadt and Louis J. Hamilton, “Nuclear Reactor Analysis,” John Wiley \& Sons, Inc. (1976).

[Hel03] HELIOS Methods, Studsvik Scandpower, 20 November 2003.

[Hon11] Ser Gi Hong, Kang Seog Kim, "Iterative Resonance Treatment Methods Using Resonance Integral Table in Heterogeneous Transport Lattice Calculations,” Ann. Nucl. Energ., 38, 32-43 (2011).

[Jam99] M. F. James, "Energy Release in Fission of Th-232, U-233, U-234, U-236, Np-237, Pu-238, Pu-240 and Pu-242," J. Nucl. Energy, 23, 1999.

[Joo09] H. G. Joo and et al., "Subgroup Weight Generation Based on Shielded Pin-Cell Cross Section Conservation,” Ann. nucl. Energy, 36, 859 (2009).

[Kim03] Kang Seog Kim et al., "Monte Carlo Resonance Treatment for the Deterministic Transport Lattice Codes,” Journal of the Korean Nuclear Society, 35, No.6, 581-595 (2003).

[Kim11] Kang-Seog Kim, Ser Gi Hong, “A New Procedure to Generate Resonance Integral Table with an Explicit Resonance Interference for Transport Lattice Codes," Ann. Nucl. Ener., 38, 118-127 (2011).

[Kim12] Kang-Seog Kim, Mark L. Williams, "Preliminary Assessment of Resonance Interference Treatment by Using 0-D Slowing Down Calculation in the Embedded Self-Shielding Method,” Trans. Am. Nucl. Soc., 107, 1128-1131 (2012).

[Lee11] C. H. Lee and W. S. Yang, "MC²-3: Multigroup Cross Section Generation Code for Fast Reactor Analysis,” ANL-NE-11-41, Argonne National Laboratory (2011).

[Liu14] Yuxuan Liu, William Martin, Mark L. Williams, Kang-Seog Kim, "Resonance Selfshielding Method for Fuel Annular Subdivisions Using Quasi-1D Slowing down Solutions," (In preparation) (2014).

[Mat05] H. Matsumoto, M. Ouisloumen and T. Takeda, "Development of Spatially Dependent Resonance Shielding Method,” J. Nucl. Sci Technol., 42, 688 (2005).

[Sca09] "SCALE: A Modular Code System for Performing Standardized Computer Analyses for Licensing Evaluation,” ORNL-TM/2005/39, Version 6, Vols. I-III, Oak Ridge National Laboratory, Oak Ridge, Tennessee (2009). (Available from Radiation Safety Information Computational Center at Oak Ridge National Laboratory as CCC-750.)

[Sta83] Rudi J.J. Stamm’ler and Maximo J. Abbate, "Methods of Steady-State Reactor Physics in Nuclear Design,” Academic Press (1983).

[Sto96] C. Stoker and Z. Weiss, "Spatially Dependent Resonance Cross Sections in a Fuel Rod,” Ann. Nucl. Energy., 23, 765 (1996).

[Wem07] C. A. Wemple, et al., "Improved Temperature-Dependent Resonance Treatment in HELIOS-1.9,” Trans. Am. Nucl. Soc., 96, 657-659 (2007).

[Wia14] D. Wiarda, S. Goluoglu, M.E. Dunn, N.M. Green and L. M. Petrie, “AMPX-6: A Modular Code System for Processing ENDF/B Evaluations,” in preparation (2014). 
[Wil06] Mark L. Williams, M. Asgari, D. F. Hollenbach, "CENTRM: A One-Dimensional Neutron Transport Code For Computing Pointwise Energy Spectra,” ORNL/TM2005/39, Version 5.1, Vol. II, Book 4, Sect. F18 (2006).

[Wil11] Mark L. Williams, "Resonance Self-Shielding Methodologies in SCALE 6," Nuclear Technology, 174, 149-168 (2011).

[Wil12] Mark L. Williams and Kang-Seog Kim, “The Embedded Self-Shielding Method,” PHYSOR 2012, Knoxville, Tennessee, USA (2012). 
Appendix A.1 Sample input of LAMBDA and IRFFACTOR-hom for ${ }^{235} \mathrm{U}$

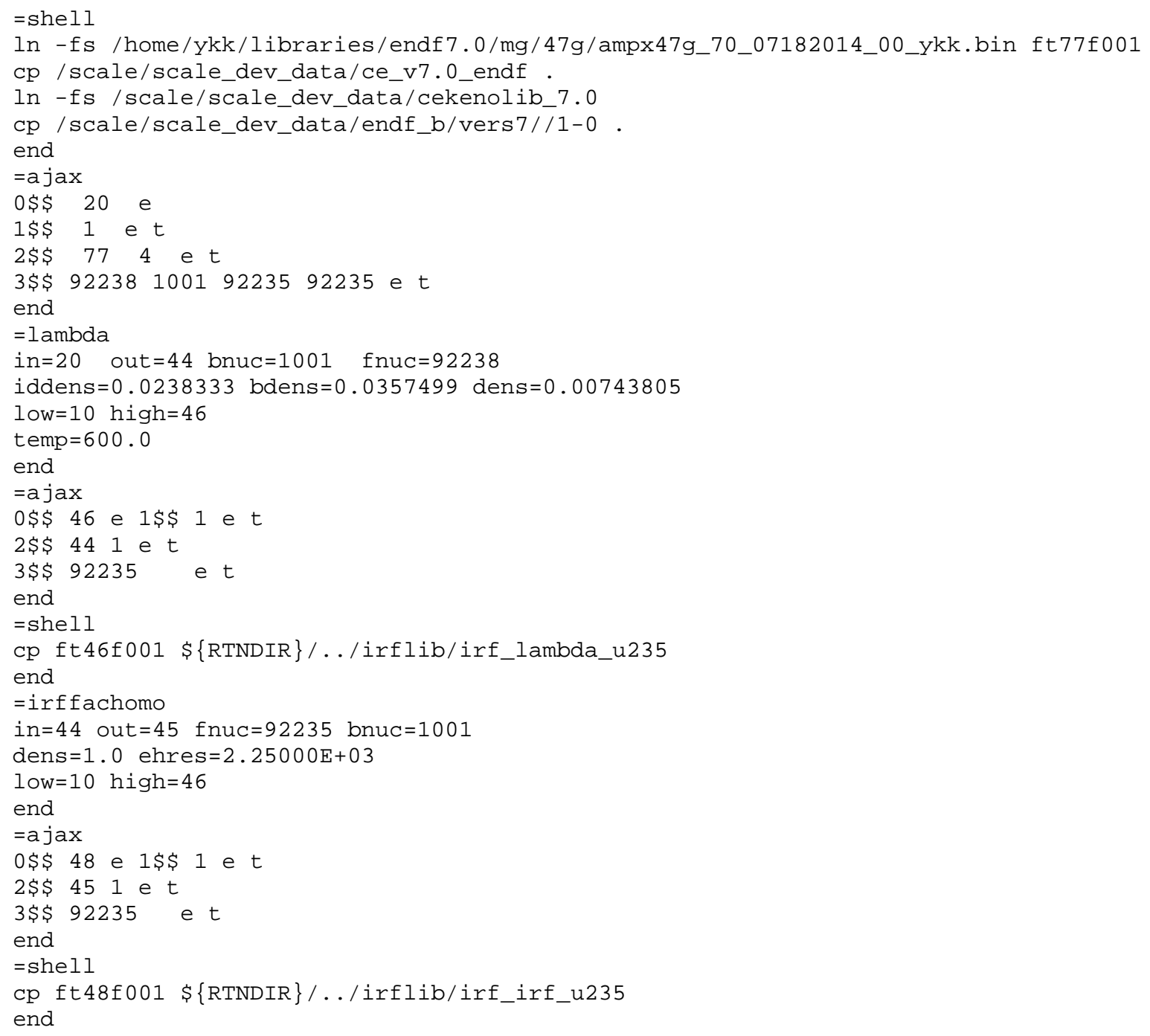


Appendix B.1 Sample input of IRFFACTOR-het for ${ }^{238} U$

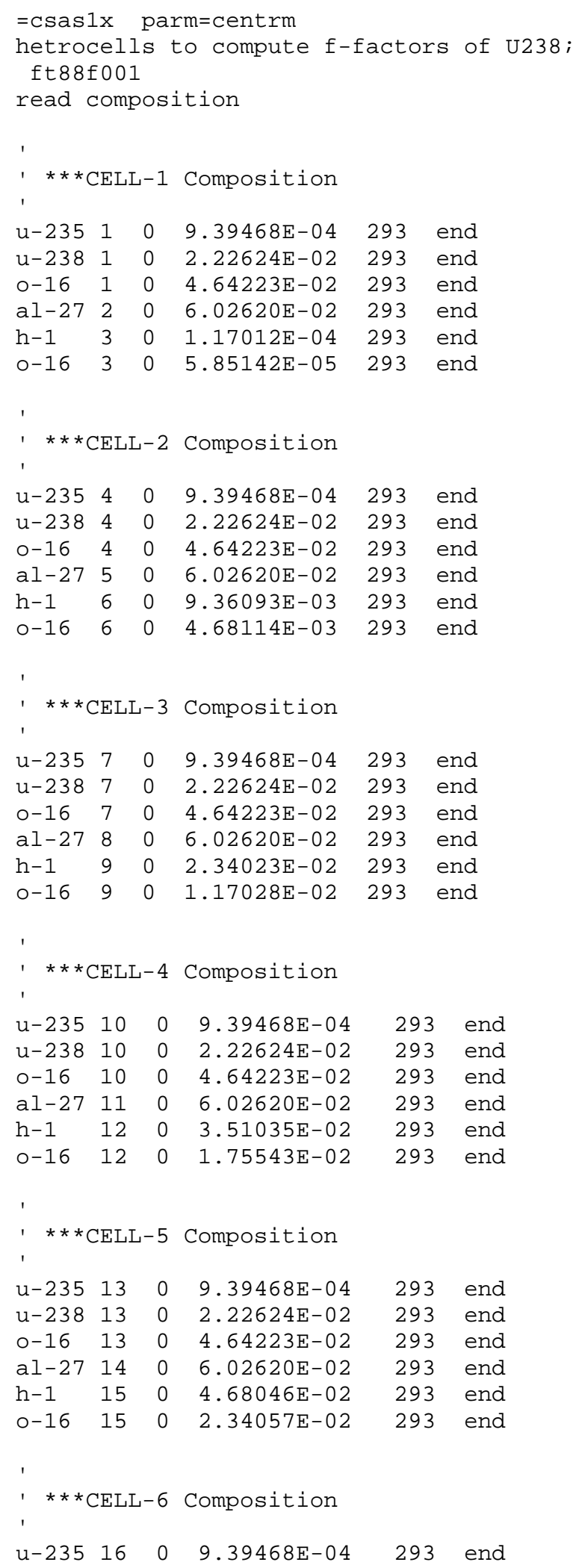




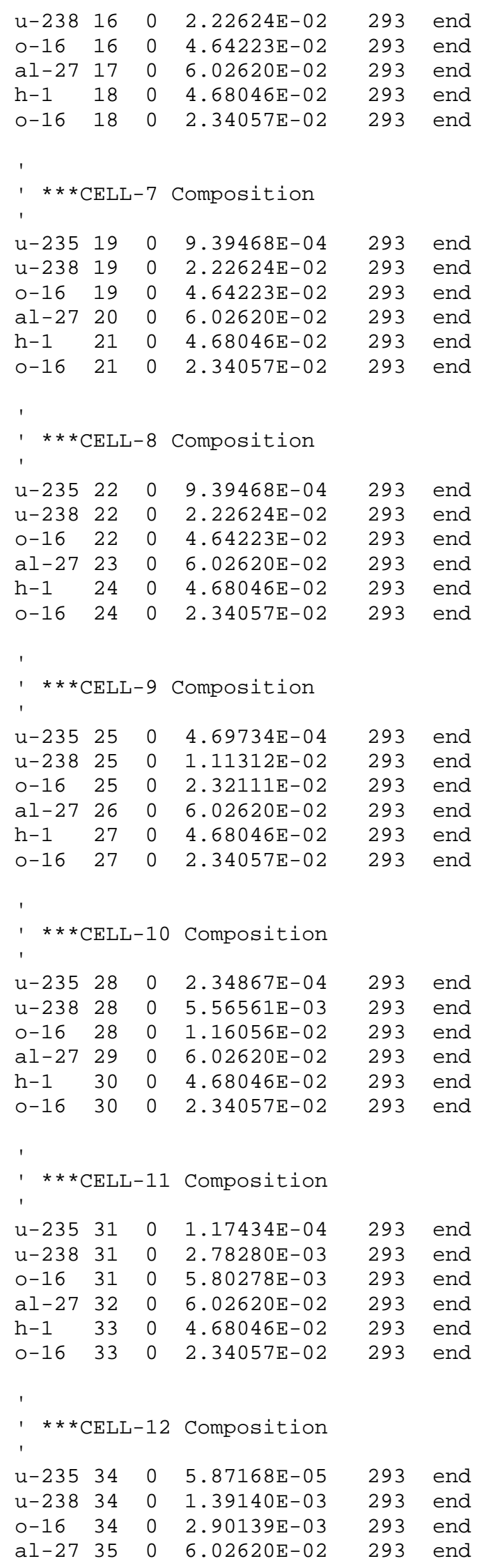



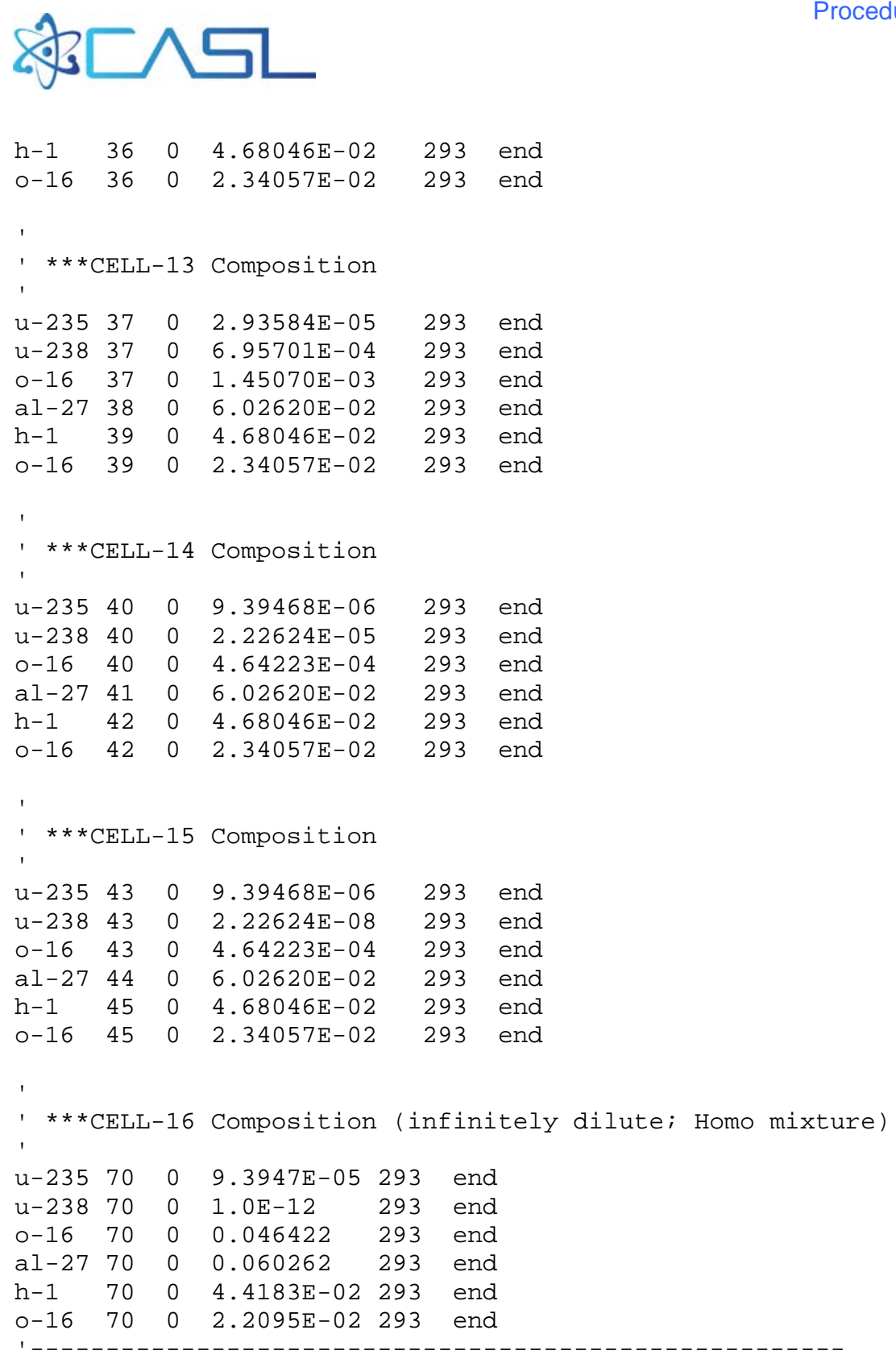

end composition

read celldata

1

' $* * *$ CELL -1 Geometry

latticecell squarepitch pitch=1.2620 3 fuelr=0.4025 1 cladr $=0.47592$ end

centrmata demin $=.0001$ demax=9. E3 iup=15 npxs=6 end centrmdata

1

' $* * *$ CELL -2 Geometry

latticecell squarepitch pitch=1.2620 6 fuelr $=0.40254$ $c l a d r=0.47595$ end

centrmata demin $=.0001$ demax $=9$. E3 $i u p=15$ npxs $=6$ end centrmdata

।

' ***CELL -3 Geometry

latticecell squarepitch pitch=1.2620 9 fuelr $=0.40257$ 


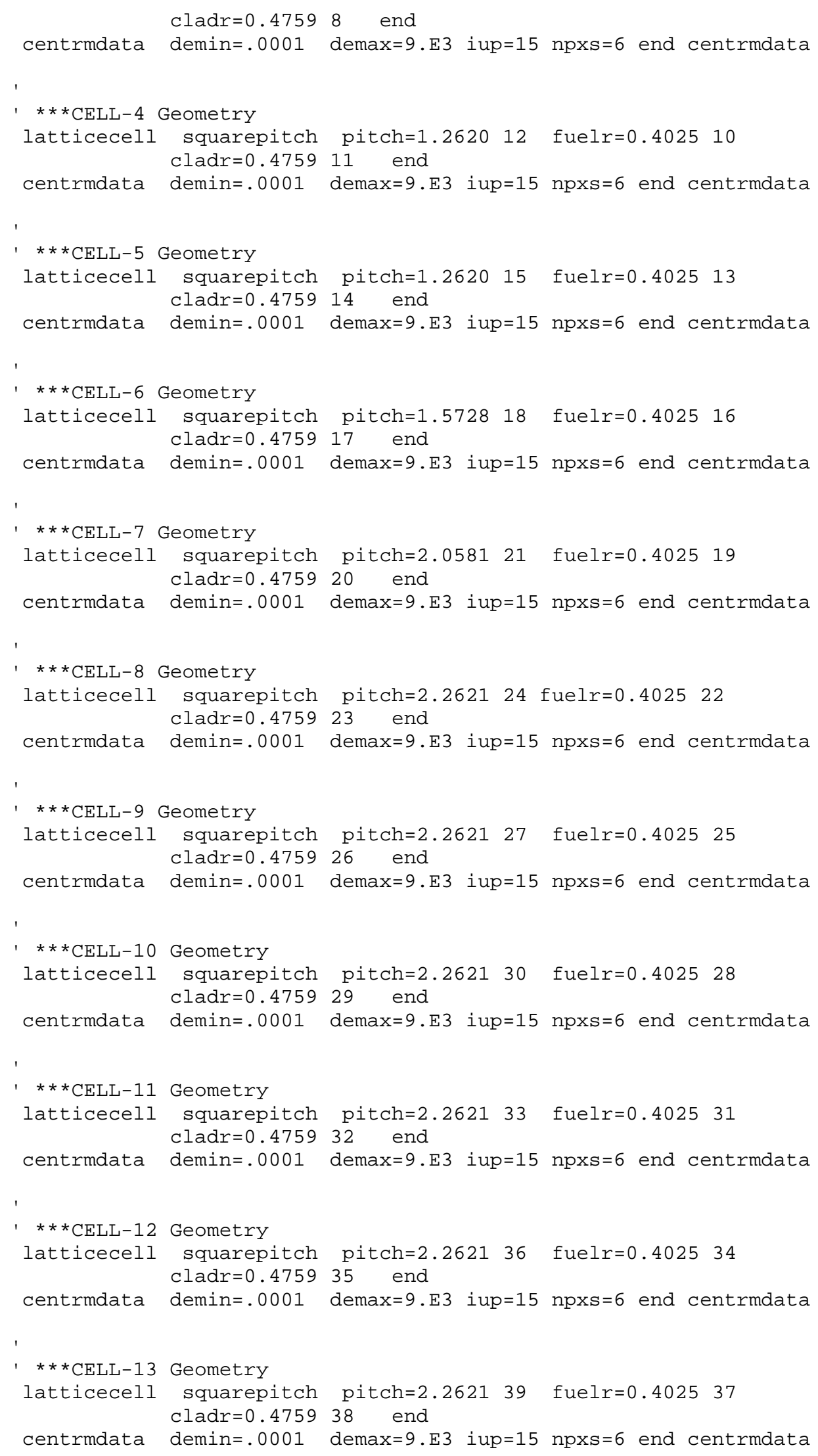






1 


\section{Appendix C.1 Data information in the MAPCT 47-group library}

NO1 0 non depletable

$>0$ depletable (w/ decay constant)

2 RI-a only (fissionable w/ kappa, beta, FPY)

3 RI -a \& RI-vf (fissionable w/ kappa, beta, FPY)

NO2 NFPY (Ordering no. of FPY) (+/- : cumulative/direct yield)

NO3 Indicator for fission spectrum $(0 />0:$ no/yes $)$

NO4 Indicator for $(n, 2 n) \&(n, 3 n)$ XS (0/1/2/3 : no/(n,2n)/(n,3n)/both)

NO5 The number of temperatures for XS

NO6 The number of temperatures for RI

NO7 The number of temperatures for $\mathrm{p} 2 \sim \mathrm{p} 3$

NO8 The number of sig-0's for RI

1 - 49: Full XS + subgroup data

50 - 191: Full XS + resonance data such as IR parameter, potential XS

192 - end: Part XS

\begin{tabular}{|c|c|c|c|c|c|c|c|c|c|c|c|}
\hline No & NID & Amass & NO1 & NO2 & NO3 & NO4 & NO5 & NO6 & NO7 & NO8 & AID \\
\hline 1 & 40091 & 90.9056 & 1 & 0 & 0 & 0 & 3 & 5 & 0 & 16 & Zr-91 \\
\hline 2 & 40096 & 95.9083 & 1 & 0 & 0 & 0 & 3 & 5 & 0 & 16 & Zr-96 \\
\hline 3 & 42595 & 94.9059 & 1 & 17 & 0 & 0 & 3 & 5 & 0 & 16 & Mo-95 \\
\hline 4 & 43599 & 99.0005 & 1 & 23 & 0 & 1 & 3 & 5 & 0 & 16 & Tc-99 \\
\hline 5 & 45103 & 102.9050 & 1 & 0 & 0 & 0 & 3 & 5 & 0 & 16 & Rh-103 \\
\hline 6 & 46608 & 107.9039 & 1 & 37 & 0 & 0 & 3 & 5 & 0 & 16 & Pd-108 \\
\hline 7 & 47107 & 106.9054 & 0 & 0 & 0 & 3 & 3 & 5 & 0 & 16 & Ag-107 \\
\hline 8 & 47109 & 108.9045 & 0 & 0 & 0 & 3 & 3 & 5 & 0 & 16 & Ag-109 \\
\hline 9 & 49113 & 112.9039 & 0 & 0 & 0 & 0 & 3 & 5 & 0 & 16 & In-113 \\
\hline 10 & 49115 & 114.9041 & 0 & 0 & 0 & 0 & 3 & 5 & 0 & 16 & In-115 \\
\hline 11 & 54631 & 130.9055 & 1 & 57 & 0 & 3 & 3 & 5 & 0 & 16 & Xe-131 \\
\hline 12 & 55633 & 132.9057 & 1 & 63 & 0 & 1 & 3 & 5 & 0 & 16 & Cs-133 \\
\hline 13 & 62152 & 151.9201 & 0 & 0 & 0 & 3 & 5 & 5 & 0 & 16 & Sm-152 \\
\hline 14 & 63151 & 150.9195 & 0 & 0 & 0 & 3 & 5 & 5 & 0 & 16 & Eu-151 \\
\hline 15 & 63152 & 151.9251 & 1 & 0 & 0 & 3 & 5 & 5 & 0 & 16 & Eu-152 \\
\hline 16 & 63153 & 152.9217 & 0 & 0 & 0 & 3 & 5 & 5 & 0 & 16 & Eu-153 \\
\hline 17 & 63154 & 153.9223 & 1 & 0 & 0 & 3 & 5 & 5 & 0 & 16 & Eu-154 \\
\hline 18 & 63155 & 154.9229 & 1 & 0 & 0 & 3 & 5 & 5 & 0 & 16 & Eu-155 \\
\hline 19 & 64155 & 154.9229 & 0 & 0 & 0 & 1 & 5 & 5 & 0 & 16 & Gd-155 \\
\hline 20 & 64156 & 155.9225 & 0 & 0 & 0 & 1 & 5 & 5 & 0 & 16 & Gd-156 \\
\hline 21 & 64157 & 156.9241 & 0 & 0 & 0 & 1 & 5 & 5 & 0 & 16 & Gd-157 \\
\hline 22 & 64158 & 157.9236 & 0 & 0 & 0 & 1 & 5 & 5 & 0 & 16 & Gd-158 \\
\hline 23 & 66160 & 159.9248 & 0 & 0 & 0 & 0 & 3 & 5 & 0 & 16 & Dy-160 \\
\hline 24 & 66161 & 160.9274 & 0 & 0 & 0 & 0 & 3 & 5 & 0 & 16 & Dy-161 \\
\hline 25 & 66162 & 161.9270 & 0 & 0 & 0 & 0 & 3 & 5 & 0 & 16 & Dy-162 \\
\hline 26 & 66163 & 162.9286 & 0 & 0 & 0 & 0 & 3 & 5 & 0 & 16 & Dy-163 \\
\hline 27 & 66164 & 163.9282 & 0 & 0 & 0 & 3 & 3 & 5 & 0 & 16 & Dy-164 \\
\hline 28 & 68166 & 165.9304 & 0 & 0 & 0 & 0 & 3 & 5 & 0 & 16 & Er-166 \\
\hline 29 & 68167 & 166.9320 & 0 & 0 & 0 & 0 & 5 & 5 & 0 & 16 & Er-167 \\
\hline 30 & 72176 & 175.9414 & 0 & 0 & 0 & 1 & 3 & 5 & 0 & 16 & Hf-176 \\
\hline 31 & 72177 & 176.9430 & 0 & 0 & 0 & 1 & 3 & 5 & 0 & 16 & Hf-177 \\
\hline 32 & 72178 & 177.9436 & 0 & 0 & 0 & 1 & 3 & 5 & 0 & 16 & Hf-178 \\
\hline 33 & 72179 & 178.9462 & 0 & 0 & 0 & 1 & 3 & 5 & 0 & 16 & Hf-179 \\
\hline 34 & 72180 & 179.9468 & 0 & 0 & 0 & 1 & 3 & 5 & 0 & 16 & Hf-180 \\
\hline 35 & 74182 & 181.9531 & 0 & 0 & 0 & 1 & 3 & 5 & 0 & 16 & W-182 \\
\hline 36 & 74183 & 182.9517 & 0 & 0 & 0 & 1 & 3 & 5 & 0 & 16 & W-183 \\
\hline 37 & 74184 & 183.9502 & 0 & 0 & 0 & 1 & 3 & 5 & 0 & 16 & W-184 \\
\hline 38 & 74186 & 185.9575 & 0 & 0 & 0 & 1 & 3 & 5 & 0 & 16 & W-186 \\
\hline
\end{tabular}




\begin{tabular}{|c|c|c|c|c|c|c|c|c|c|c|c|}
\hline 39 & 90232 & 232.0333 & 2 & 0 & 1 & 3 & 5 & 5 & 5 & 16 & Th-232 \\
\hline 40 & 92233 & 233.0450 & 3 & 0 & 2 & 3 & 5 & 5 & 5 & 16 & U-233 \\
\hline 41 & 92235 & 235.0441 & 3 & 0 & 3 & 3 & 5 & 5 & 5 & 16 & U-235 \\
\hline 42 & 92236 & 236.0458 & 3 & 0 & 4 & 3 & 3 & 5 & 3 & 16 & U-236 \\
\hline 43 & 92238 & 238.0510 & 2 & 0 & 5 & 3 & 5 & 5 & 5 & 16 & U-238 \\
\hline 44 & 94238 & 238.0495 & 3 & 0 & 6 & 3 & 3 & 5 & 0 & 16 & $\mathrm{Pu}-238$ \\
\hline 45 & 94239 & 239.0522 & 3 & 0 & 7 & 3 & 5 & 5 & 5 & 16 & Pu-239 \\
\hline 46 & 94240 & 240.0542 & 3 & 0 & 8 & 3 & 5 & 5 & 5 & 16 & $\mathrm{Pu}-240$ \\
\hline 47 & 94241 & 241.0487 & 3 & 0 & 9 & 3 & 5 & 5 & 5 & 16 & Pu-241 \\
\hline 48 & 94242 & 242.0584 & 3 & 0 & 10 & 3 & 3 & 5 & 3 & 16 & $\mathrm{Pu}-242$ \\
\hline 49 & 95241 & 241.0568 & 3 & 0 & 0 & 3 & 3 & 5 & 0 & 16 & Am-241 \\
\hline 50 & 1001 & 1.0078 & 0 & 0 & 0 & 0 & 8 & 0 & 8 & 0 & $\mathrm{H}-1$ \\
\hline 51 & 1002 & 2.0141 & 0 & 0 & 0 & 1 & 5 & 0 & 5 & 0 & H-2 \\
\hline 52 & 1006 & 1.0078 & 0 & 0 & 0 & 0 & 3 & 0 & 3 & 0 & $\mathrm{H}-1$ \\
\hline 53 & 1040 & 1.0078 & 0 & 0 & 0 & 0 & 3 & 0 & 3 & 0 & $\mathrm{H}-1$ \\
\hline 54 & 3006 & 4.0026 & 0 & 0 & 0 & 0 & 5 & 0 & 5 & 0 & Li-6 \\
\hline 55 & 3007 & 6.0151 & 0 & 0 & 0 & 1 & 3 & 0 & 3 & 0 & $\mathrm{Li}-7$ \\
\hline 56 & 4009 & 7.0160 & 0 & 0 & 0 & 1 & 3 & 0 & 0 & 0 & Be-9 \\
\hline 57 & 5000 & 9.0122 & 0 & 0 & 0 & 0 & 3 & 0 & 0 & 0 & B-Nat \\
\hline 58 & 5010 & 10.8120 & 1 & 0 & 0 & 0 & 3 & 0 & 0 & 0 & B-10 \\
\hline 59 & 5011 & 10.0129 & 1 & 0 & 0 & 0 & 3 & 0 & 0 & 0 & B-11 \\
\hline 60 & 6000 & 11.0093 & 0 & 0 & 0 & 0 & 5 & 0 & 5 & 0 & C-Nat \\
\hline 61 & 6001 & 12.0011 & 0 & 0 & 0 & 0 & 10 & 0 & 10 & 0 & C-Nat \\
\hline 62 & 7014 & 12.0011 & 0 & 0 & 0 & 1 & 3 & 0 & 0 & 0 & $\mathrm{~N}-14$ \\
\hline 63 & 8001 & 14.0031 & 0 & 0 & 0 & 0 & 5 & 0 & 5 & 0 & O-UO2 \\
\hline 64 & 8016 & 15.9905 & 0 & 0 & 0 & 0 & 5 & 0 & 5 & 0 & O-16 \\
\hline 65 & 9019 & 15.9905 & 0 & 0 & 0 & 0 & 3 & 0 & 0 & 0 & F-19 \\
\hline 66 & 11023 & 18.9982 & 0 & 0 & 0 & 0 & 5 & 0 & 0 & 0 & Na-23 \\
\hline 67 & 12000 & 22.9895 & 0 & 0 & 0 & 0 & 3 & 0 & 0 & 0 & Mg-Nat \\
\hline 68 & 13027 & 24.3051 & 0 & 0 & 0 & 0 & 3 & 0 & 3 & 0 & Al-27 \\
\hline 69 & 14000 & 26.9818 & 0 & 0 & 0 & 0 & 3 & 0 & 0 & 0 & Si-Nat \\
\hline 70 & 15031 & 28.0853 & 0 & 0 & 0 & 0 & 3 & 0 & 0 & 0 & P-31 \\
\hline 71 & 16000 & 30.9741 & 0 & 0 & 0 & 0 & 3 & 0 & 0 & 0 & S-Nat \\
\hline 72 & 17000 & 32.0636 & 0 & 0 & 0 & 0 & 3 & 0 & 0 & 0 & Cl-Nat \\
\hline 73 & 19000 & 35.4526 & 0 & 0 & 0 & 0 & 3 & 0 & 0 & 0 & K-Nat \\
\hline 74 & 20000 & 39.1019 & 0 & 0 & 0 & 0 & 3 & 0 & 0 & 0 & Ca-Nat \\
\hline 75 & 22000 & 40.0803 & 0 & 0 & 0 & 0 & 3 & 0 & 0 & 0 & Ti-Nat \\
\hline 76 & 23000 & 47.8789 & 0 & 0 & 0 & 0 & 3 & 0 & 0 & 0 & V-Nat \\
\hline 77 & 24000 & 50.9416 & 0 & 0 & 0 & 1 & 3 & 0 & 0 & 0 & Cr-Nat \\
\hline 78 & 24050 & 51.9957 & 0 & 0 & 0 & 0 & 3 & 0 & 0 & 0 & Cr-50 \\
\hline 79 & 24052 & 49.9461 & 0 & 0 & 0 & 0 & 3 & 0 & 0 & 0 & Cr-52 \\
\hline 80 & 24053 & 51.9402 & 0 & 0 & 0 & 0 & 3 & 0 & 0 & 0 & Cr-53 \\
\hline 81 & 24054 & 52.9408 & 0 & 0 & 0 & 0 & 3 & 0 & 0 & 0 & Cr-54 \\
\hline 82 & 25055 & 53.9394 & 0 & 0 & 0 & 1 & 3 & 0 & 0 & 0 & Mn-55 \\
\hline 83 & 26000 & 54.9381 & 0 & 0 & 0 & 1 & 3 & 0 & 0 & 0 & Fe-Nat \\
\hline 84 & 26054 & 55.8446 & 0 & 0 & 0 & 0 & 3 & 0 & 0 & 0 & Fe-54 \\
\hline 85 & 26056 & 53.9394 & 0 & 0 & 0 & 0 & 3 & 0 & 0 & 0 & Fe-56 \\
\hline 86 & 26057 & 55.9345 & 0 & 0 & 0 & 0 & 3 & 0 & 0 & 0 & Fe-57 \\
\hline 87 & 26058 & 56.9351 & 0 & 0 & 0 & 0 & 3 & 0 & 0 & 0 & Fe-58 \\
\hline 88 & 27059 & 57.9337 & 0 & 0 & 0 & 1 & 3 & 0 & 0 & 0 & Co-59 \\
\hline 89 & 28000 & 58.9332 & 0 & 0 & 0 & 1 & 3 & 0 & 0 & 0 & Ni-Nat \\
\hline 90 & 28058 & 58.6936 & 0 & 0 & 0 & 0 & 3 & 0 & 0 & 0 & $\mathrm{Ni}-58$ \\
\hline 91 & 28060 & 57.9357 & 0 & 0 & 0 & 0 & 3 & 0 & 0 & 0 & $\mathrm{Ni}-60$ \\
\hline 92 & 28061 & 59.9308 & 0 & 0 & 0 & 0 & 3 & 0 & 0 & 0 & $\mathrm{Ni}-61$ \\
\hline 93 & 28062 & 60.9314 & 0 & 0 & 0 & 0 & 3 & 0 & 0 & 0 & $\mathrm{Ni}-62$ \\
\hline 94 & 28064 & 61.9280 & 0 & 0 & 0 & 0 & 3 & 0 & 0 & 0 & Ni-64 \\
\hline 95 & 29063 & 63.9282 & 0 & 0 & 0 & 0 & 3 & 0 & 0 & 0 & $\mathrm{Cu}-63$ \\
\hline 96 & 29065 & 62.9296 & 0 & 0 & 0 & 0 & 3 & 0 & 0 & 0 & Cu-65 \\
\hline 97 & 40000 & 64.9278 & 0 & 0 & 0 & 1 & 3 & 0 & 3 & 0 & Zr-Nat \\
\hline
\end{tabular}




\begin{tabular}{|c|c|c|c|c|c|c|c|c|c|c|c|}
\hline 98 & 40001 & 91.2196 & 0 & 0 & 0 & 1 & 8 & 0 & 8 & 0 & Zr-Zrh2 \\
\hline 99 & 40090 & 91.2196 & 0 & 0 & 0 & 0 & 3 & 0 & 0 & 0 & Zr-90 \\
\hline 100 & 40092 & 89.9043 & 0 & 0 & 0 & 0 & 3 & 0 & 0 & 0 & Zr-92 \\
\hline 101 & 40094 & 91.9050 & 0 & 0 & 0 & 0 & 3 & 0 & 0 & 0 & Zr-94 \\
\hline 102 & 41093 & 93.9063 & 0 & 0 & 0 & 0 & 5 & 0 & 0 & 0 & Nb-93 \\
\hline 103 & 42000 & 92.9032 & 0 & 0 & 0 & 3 & 3 & 0 & 0 & 0 & Mo-Nat \\
\hline 104 & 44601 & 95.9402 & 1 & 25 & 0 & 0 & 3 & 0 & 0 & 0 & Ru-101 \\
\hline 105 & 45103 & 100.9058 & 0 & 0 & 0 & 0 & 3 & 0 & 0 & 0 & Rh-103 \\
\hline 106 & 45603 & $\begin{array}{l}102.9050 \\
\end{array}$ & 1 & 31 & 0 & 1 & 3 & 0 & 0 & 0 & Rh-103 \\
\hline 107 & 45605 & 104.9062 & 1 & 32 & 0 & 0 & 3 & 0 & 0 & 0 & Rh-105 \\
\hline 108 & 46605 & 104.9052 & 1 & 34 & 0 & 0 & 3 & 0 & 0 & 0 & Pd-105 \\
\hline 109 & 46607 & 106.9054 & 1 & 36 & 0 & 0 & 3 & 0 & 0 & 0 & Pd-107 \\
\hline 110 & 47609 & 108.9045 & 1 & 38 & 0 & 3 & 3 & 0 & 0 & 0 & Ag-109 \\
\hline 111 & 48000 & 112.4258 & 0 & 0 & 0 & 0 & 3 & 0 & 0 & 0 & Cd-Nat \\
\hline 112 & 48110 & 109.9031 & 0 & 0 & 0 & 0 & 3 & 0 & 0 & 0 & Cd-110 \\
\hline 113 & 48111 & 110.9047 & 0 & 0 & 0 & 0 & 3 & 0 & 0 & 0 & Cd-111 \\
\hline 114 & 48112 & 111.9033 & 0 & 0 & 0 & 0 & 3 & 0 & 0 & 0 & Cd-112 \\
\hline 115 & 48113 & 112.8999 & 0 & 0 & 0 & 1 & 3 & 0 & 0 & 0 & Cd-113 \\
\hline 116 & 48114 & 113.9035 & 0 & 0 & 0 & 0 & 3 & 0 & 0 & 0 & Cd-114 \\
\hline 117 & 49000 & 114.9071 & 0 & 0 & 0 & 0 & 3 & 0 & 0 & 0 & In-Nat \\
\hline 118 & 49615 & 114.9071 & 1 & 44 & 0 & 0 & 3 & 0 & 0 & 0 & In-115 \\
\hline 119 & 50000 & 118.7107 & 0 & 0 & 0 & 0 & 3 & 0 & 0 & 0 & Sn-Nat \\
\hline 120 & 50112 & 111.9013 & 0 & 0 & 0 & 0 & 3 & 0 & 0 & 0 & Sn-112 \\
\hline 121 & 50114 & 113.8984 & 0 & 0 & 0 & 0 & 3 & 0 & 0 & 0 & Sn-114 \\
\hline 122 & 50115 & 114.9031 & 0 & 0 & 0 & 0 & 3 & 0 & 0 & 0 & Sn-115 \\
\hline 123 & 50116 & 115.9017 & 0 & 0 & 0 & 0 & 3 & 0 & 0 & 0 & Sn-116 \\
\hline 124 & 50117 & 116.9033 & 0 & 0 & 0 & 0 & 3 & 0 & 0 & 0 & Sn-117 \\
\hline 125 & 50118 & 117.9018 & 0 & 0 & 0 & 0 & 3 & 0 & 0 & 0 & Sn-118 \\
\hline 126 & 50119 & 118.9035 & 0 & 0 & 0 & 0 & 3 & 0 & 0 & 0 & Sn-119 \\
\hline 127 & 50120 & 119.9020 & 0 & 0 & 0 & 0 & 3 & 0 & 0 & 0 & Sn-120 \\
\hline 128 & 50122 & 121.9032 & 0 & 0 & 0 & 0 & 3 & 0 & 0 & 0 & Sn-122 \\
\hline 129 & 50124 & 123.9054 & 0 & 0 & 0 & 0 & 3 & 0 & 0 & 0 & Sn-124 \\
\hline 130 & 51000 & 121.7577 & 0 & 0 & 0 & 0 & 3 & 0 & 0 & 0 & Sb-Nat \\
\hline 131 & 51121 & 120.9036 & 0 & 0 & 0 & 0 & 3 & 0 & 0 & 0 & Sb-121 \\
\hline 132 & 51123 & 122.9038 & 0 & 0 & 0 & 0 & 3 & 0 & 0 & 0 & Sb-123 \\
\hline 133 & 54634 & 133.9053 & 1 & 60 & 0 & 3 & 3 & 0 & 0 & 0 & Xe-134 \\
\hline 134 & 60643 & 142.9097 & 1 & 81 & 0 & 3 & 3 & 0 & 0 & 0 & Nd-143 \\
\hline 135 & 60645 & 144.9129 & 1 & 83 & 0 & 3 & 3 & 0 & 0 & 0 & Nd-145 \\
\hline 136 & 61647 & 146.9151 & 1 & 88 & 0 & 3 & 3 & 0 & 0 & 0 & Pm-147 \\
\hline 137 & 61748 & 147.9177 & 1 & 92 & 0 & 0 & 3 & 0 & 0 & 0 & Pm-148m \\
\hline 138 & 62153 & 152.9217 & 1 & 0 & 0 & 0 & 3 & 0 & 0 & 0 & Sm-153 \\
\hline 139 & 62647 & 146.9151 & 1 & 93 & 0 & 3 & 3 & 0 & 0 & 0 & Sm-147 \\
\hline 140 & 62649 & 148.9173 & 1 & 95 & 0 & 3 & 3 & 0 & 0 & 0 & Sm-149 \\
\hline 141 & 62650 & 149.9169 & 1 & 96 & 0 & 1 & 3 & 0 & 0 & 0 & Sm-150 \\
\hline 142 & 62651 & 150.9195 & 1 & 97 & 0 & 3 & 3 & 0 & 0 & 0 & Sm-151 \\
\hline 143 & 62652 & 151.9201 & 1 & 98 & 0 & 3 & 3 & 0 & 0 & 0 & Sm-152 \\
\hline 144 & 63156 & 155.9245 & 1 & 0 & 0 & 0 & 3 & 0 & 0 & 0 & Eu-156 \\
\hline 145 & 63157 & 156.9251 & 1 & 0 & 0 & 0 & 3 & 0 & 0 & 0 & Eu-157 \\
\hline 146 & 63653 & 152.9217 & 1 & 102 & 0 & 3 & 3 & 0 & 0 & 0 & Eu-153 \\
\hline 147 & 63654 & 153.9223 & 1 & 103 & 0 & 3 & 3 & 0 & 0 & 0 & Eu-154 \\
\hline 148 & 63655 & 154.9229 & 1 & 104 & 0 & 3 & 3 & 0 & 0 & 0 & Eu-155 \\
\hline 149 & 64152 & 151.9201 & 0 & 0 & 0 & 1 & 3 & 0 & 0 & 0 & Gd-152 \\
\hline 150 & 64154 & 153.9213 & 0 & 0 & 0 & 1 & 3 & 0 & 0 & 0 & Gd-154 \\
\hline 151 & 64160 & 159.9268 & 0 & 0 & 0 & 1 & 3 & 0 & 0 & 0 & Gd-160 \\
\hline 152 & 64655 & 154.9229 & 1 & 108 & 0 & 1 & 3 & 0 & 0 & 0 & Gd-155 \\
\hline 153 & 64656 & 155.9225 & 1 & 109 & 0 & 1 & 3 & 0 & 0 & 0 & Gd-156 \\
\hline 154 & 64657 & 156.9241 & 1 & 110 & 0 & 1 & 3 & 0 & 0 & 0 & Gd-157 \\
\hline 155 & 64658 & 157.9236 & 1 & 111 & 0 & 1 & 3 & 0 & 0 & 0 & Gd-158 \\
\hline 156 & 68168 & 167.9414 & 0 & 0 & 0 & 0 & 3 & 0 & 0 & 0 & Er-166 \\
\hline
\end{tabular}




\begin{tabular}{|c|c|c|c|c|c|c|c|c|c|c|c|}
\hline 157 & 71176 & 175.9414 & 0 & 0 & 0 & 0 & 3 & 0 & 0 & 0 & $\begin{array}{l}\text { Lu-176 } \\
\end{array}$ \\
\hline 158 & 72174 & 173.9402 & 0 & 0 & 0 & 1 & 3 & 0 & 0 & 0 & Hf-174 \\
\hline 159 & 73181 & 180.9545 & 0 & 0 & 0 & 3 & 3 & 0 & 0 & 0 & Ta-181 \\
\hline 160 & 73182 & 181.9500 & 0 & 0 & 0 & 3 & 3 & 0 & 0 & 0 & Ta-182 \\
\hline 161 & 74000 & 183.8564 & 0 & 0 & 0 & 3 & 3 & 0 & 0 & 0 & W-Nat \\
\hline 162 & 79197 & $\begin{array}{l}196.9660 \\
\end{array}$ & 0 & 0 & 0 & 0 & 3 & 0 & 0 & 0 & $\mathrm{Au}-197$ \\
\hline 163 & 82206 & 205.9694 & 0 & 0 & 0 & 0 & 3 & 0 & 0 & 0 & Pb-206 \\
\hline 164 & 82207 & 206.9780 & 0 & 0 & 0 & 0 & 3 & 0 & 0 & 0 & Pb-207 \\
\hline 165 & 82208 & 207.9766 & 0 & 0 & 0 & 0 & 3 & 0 & 0 & 0 & Pb-208 \\
\hline 166 & 83209 & 208.9803 & 0 & 0 & 0 & 3 & 3 & 0 & 0 & 0 & Bi-209 \\
\hline 167 & 90230 & 230.0361 & 3 & 0 & 0 & 3 & 3 & 0 & 0 & 0 & Th-230 \\
\hline 168 & 91231 & 231.0347 & 3 & 0 & 0 & 3 & 3 & 0 & 0 & 0 & Pa-231 \\
\hline 169 & 91233 & 233.0399 & 3 & 0 & 0 & 3 & 5 & 0 & 0 & 0 & Pa-233 \\
\hline 170 & 92232 & 232.0333 & 3 & 0 & 0 & 3 & 3 & 0 & 0 & 0 & U-232 \\
\hline 171 & 92234 & 234.0405 & 3 & 0 & 11 & 3 & 3 & 0 & 3 & 0 & U-234 \\
\hline 172 & 92237 & 237.0484 & 3 & 0 & 0 & 3 & 3 & 0 & 0 & 0 & U-237 \\
\hline 173 & 93237 & 237.0482 & 3 & 0 & 12 & 3 & 3 & 0 & 0 & 0 & Np-237 \\
\hline 174 & 93238 & 238.0510 & 3 & 0 & 0 & 0 & 3 & 0 & 0 & 0 & Np-238 \\
\hline 175 & 93239 & 239.0526 & 3 & 0 & 13 & 3 & 3 & 0 & 0 & 0 & Np-239 \\
\hline 176 & 94236 & 236.0458 & 3 & 0 & 14 & 3 & 3 & 0 & 0 & 0 & Pu-236 \\
\hline 177 & 95242 & 242.0594 & 3 & 0 & 0 & 0 & 3 & 0 & 0 & 0 & Am-242 \\
\hline 178 & 95342 & 242.0594 & 3 & 0 & 0 & 3 & 3 & 0 & 0 & 0 & Am-242m \\
\hline 179 & 95243 & 243.0610 & 3 & 0 & 0 & 3 & 3 & 0 & 0 & 0 & Am-243 \\
\hline 180 & 96242 & 242.0584 & 3 & 0 & 0 & 3 & 3 & 0 & 0 & 0 & Cm-242 \\
\hline 181 & 96243 & 243.0610 & 3 & 0 & 0 & 3 & 3 & 0 & 0 & 0 & $\mathrm{Cm}-243$ \\
\hline 182 & 96244 & 244.0626 & 3 & 0 & 0 & 3 & 3 & 0 & 0 & 0 & Cm-244 \\
\hline 183 & 96245 & 245.0652 & 3 & 0 & 0 & 3 & 3 & 0 & 0 & 0 & Cm-245 \\
\hline 184 & 96246 & 246.0668 & 3 & 0 & 0 & 3 & 3 & 0 & 0 & 0 & Cm-246 \\
\hline 185 & 96247 & 247.0725 & 3 & 0 & 0 & 3 & 3 & 0 & 0 & 0 & Cm-247 \\
\hline 186 & 96248 & 248.0721 & 3 & 0 & 0 & 3 & 3 & 0 & 0 & 0 & Cm-248 \\
\hline 187 & 97249 & 249.0797 & 3 & 0 & 0 & 3 & 3 & 0 & 0 & 0 & Bk-249 \\
\hline 188 & 98249 & 249.0797 & 3 & 0 & 0 & 3 & 3 & 0 & 0 & 0 & Cf-249 \\
\hline 189 & 98250 & 250.0763 & 3 & 0 & 0 & 3 & 3 & 0 & 0 & 0 & Cf-250 \\
\hline 190 & 98251 & 251.0799 & 3 & 0 & 0 & 3 & 3 & 0 & 0 & 0 & Cf-251 \\
\hline 191 & 98252 & 252.0815 & 3 & 0 & 0 & 3 & 3 & 0 & 0 & 0 & Cf-252 \\
\hline 192 & 1003 & 3.0160 & 0 & 0 & 0 & 0 & 3 & 0 & 0 & 0 & T-3 \\
\hline 193 & 2003 & 3.0149 & 0 & 0 & 0 & 0 & 3 & 0 & 0 & 0 & He-3 \\
\hline 194 & 35581 & 80.9163 & 1 & 1 & 0 & 0 & 1 & 0 & 0 & 0 & Br-81 \\
\hline 195 & 36582 & 81.9135 & 1 & 2 & 0 & 1 & 1 & 0 & 0 & 0 & Kr-82 \\
\hline 196 & 36583 & 82.9141 & 1 & 3 & 0 & 3 & 1 & 0 & 0 & 0 & Kr-83 \\
\hline 197 & 36584 & 83.9114 & 1 & 4 & 0 & 1 & 1 & 0 & 0 & 0 & Kr-84 \\
\hline 198 & 36585 & 84.9125 & 1 & 5 & 0 & 0 & 1 & 0 & 0 & 0 & Kr-85 \\
\hline 199 & 36586 & 85.9106 & 1 & 6 & 0 & 3 & 1 & 0 & 0 & 0 & Kr-86 \\
\hline 200 & 38589 & 88.9075 & 1 & 7 & 0 & 0 & 1 & 0 & 0 & 0 & Sr-89 \\
\hline 201 & 38590 & 89.9078 & 1 & 8 & 0 & 0 & 1 & 0 & 0 & 0 & Sr-90 \\
\hline 202 & 39589 & 88.9058 & 1 & 9 & 0 & 1 & 1 & 0 & 0 & 0 & Y-89 \\
\hline 203 & 39590 & 89.9072 & 1 & 10 & 0 & 0 & 1 & 0 & 0 & 0 & Y-90 \\
\hline 204 & 39591 & 90.9074 & 1 & 11 & 0 & 0 & 1 & 0 & 0 & 0 & Y-91 \\
\hline 205 & 40591 & 90.9029 & 1 & 12 & 0 & 1 & 1 & 0 & 0 & 0 & Zr-91 \\
\hline 206 & 40593 & 92.9064 & 1 & 13 & 0 & 0 & 1 & 0 & 0 & 0 & Zr-93 \\
\hline 207 & 40595 & 94.9080 & 1 & 14 & 0 & 0 & 1 & 0 & 0 & 0 & Zr-95 \\
\hline 208 & 40596 & $\begin{array}{l}95.9049 \\
\end{array}$ & 1 & 15 & 0 & 1 & 1 & 0 & 0 & 0 & Zr-96 \\
\hline 209 & 41595 & 94.9068 & 1 & 16 & 0 & 0 & 1 & 0 & 0 & 0 & Nb-95 \\
\hline 210 & 42095 & 94.9059 & 0 & 0 & 0 & 0 & 1 & 0 & 0 & 0 & Mo-95 \\
\hline 211 & 42596 & 95.9047 & 1 & 18 & 0 & 0 & 3 & 0 & 0 & 0 & Mo-96 \\
\hline 212 & 42597 & 96.9061 & 1 & 19 & 0 & 0 & 1 & 0 & 0 & 0 & Mo-97 \\
\hline 213 & 42598 & 97.9055 & 1 & 20 & 0 & 0 & 1 & 0 & 0 & 0 & Mo-98 \\
\hline 214 & 42599 & 98.9078 & 1 & 21 & 0 & 0 & 1 & 0 & 0 & 0 & Mo-99 \\
\hline 215 & 42600 & 99.9075 & 1 & 22 & 0 & 0 & 1 & 0 & 0 & 0 & Mo-100 \\
\hline
\end{tabular}




\begin{tabular}{|c|c|c|c|c|c|c|c|c|c|c|c|}
\hline 216 & 44600 & 99.9042 & 1 & 24 & 0 & 0 & 1 & 0 & 0 & 0 & Ru-100 \\
\hline 217 & 44602 & 101.9044 & 1 & 26 & 0 & 0 & 1 & 0 & 0 & 0 & Ru-102 \\
\hline 218 & 44603 & 102.9060 & 1 & 27 & 0 & 0 & 1 & 0 & 0 & 0 & Ru-103 \\
\hline 219 & 44604 & 103.9056 & 1 & 28 & 0 & 0 & 1 & 0 & 0 & 0 & Ru-104 \\
\hline 220 & 44605 & 104.9082 & 1 & 29 & 0 & 0 & 1 & 0 & 0 & 0 & Ru-105 \\
\hline 221 & 44606 & 105.9078 & 1 & 30 & 0 & 0 & 1 & 0 & 0 & 0 & Ru-106 \\
\hline 222 & 46604 & 103.9036 & 1 & 33 & 0 & 0 & 1 & 0 & 0 & 0 & Pd-104 \\
\hline 223 & 46606 & 105.9038 & 1 & 35 & 0 & 0 & 1 & 0 & 0 & 0 & Pd-106 \\
\hline 224 & 47710 & $\begin{array}{l}109.9061 \\
\end{array}$ & 1 & 39 & 0 & 0 & 1 & 0 & 0 & 0 & Ag-110m \\
\hline 225 & 47611 & 110.9057 & 1 & 40 & 0 & 0 & 1 & 0 & 0 & 0 & Ag-111 \\
\hline 226 & 48610 & 109.9031 & 1 & 41 & 0 & 0 & 1 & 0 & 0 & 0 & Cd-110 \\
\hline 227 & 48611 & 110.9047 & 1 & 42 & 0 & 0 & 1 & 0 & 0 & 0 & Cd-111 \\
\hline 228 & 48613 & 112.8999 & 1 & 43 & 0 & 1 & 1 & 0 & 0 & 0 & Cd-113 \\
\hline 229 & 50125 & 124.9080 & 0 & 0 & 0 & 0 & 1 & 0 & 0 & 0 & Sn-125 \\
\hline 230 & 51621 & 120.9087 & 1 & 45 & 0 & 0 & 1 & 0 & 0 & 0 & Sb-121 \\
\hline 231 & 51625 & 124.9050 & 1 & 46 & 0 & 0 & 1 & 0 & 0 & 0 & Sb-125 \\
\hline 232 & 51627 & 126.9069 & 1 & 47 & 0 & 0 & 1 & 0 & 0 & 0 & Sb-127 \\
\hline 233 & 52727 & 126.9052 & 1 & 48 & 0 & 0 & 1 & 0 & 0 & 0 & Te-127m \\
\hline 234 & 52729 & 128.9074 & 1 & 49 & 0 & 0 & 1 & 0 & 0 & 0 & Te-129m \\
\hline 235 & 52632 & 131.9082 & 1 & 50 & 0 & 0 & 1 & 0 & 0 & 0 & Te-132 \\
\hline 236 & 53627 & 126.9042 & 1 & 51 & 0 & 3 & 1 & 0 & 0 & 0 & I-127 \\
\hline 237 & 53629 & 128.9054 & 1 & 52 & 0 & 0 & 1 & 0 & 0 & 0 & I-129 \\
\hline 238 & 53631 & 130.9066 & 1 & 53 & 0 & 0 & 1 & 0 & 0 & 0 & I-131 \\
\hline 239 & 53635 & 134.9100 & 1 & 54 & 0 & 0 & 1 & 0 & 0 & 0 & I-135 \\
\hline 240 & 54628 & 127.9038 & 1 & 55 & 0 & 3 & 1 & 0 & 0 & 0 & Xe-128 \\
\hline 241 & 54630 & 129.9039 & 1 & 56 & 0 & 3 & 1 & 0 & 0 & 0 & Xe-130 \\
\hline 242 & 54632 & 131.9041 & 1 & 58 & 0 & 3 & 1 & 0 & 0 & 0 & Хe-132 \\
\hline 243 & 54633 & 132.9057 & 1 & 59 & 0 & 0 & 1 & 0 & 0 & 0 & Xe-133 \\
\hline 244 & 54635 & 134.9069 & 1 & 61 & 0 & 0 & 1 & 0 & 0 & 0 & Xe-135 \\
\hline 245 & 54636 & 135.9075 & 1 & 62 & 0 & 3 & 1 & 0 & 0 & 0 & Хe-136 \\
\hline 246 & 55634 & 133.9073 & 1 & 64 & 0 & 0 & $\frac{x}{1}$ & 0 & 0 & 0 & Cs-134 \\
\hline 247 & 55635 & 134.9059 & 1 & 65 & 0 & 0 & 1 & 0 & 0 & 0 & Cs-135 \\
\hline 248 & 55636 & 135.9075 & 1 & 66 & 0 & 0 & 1 & 0 & 0 & 0 & Cs-136 \\
\hline 249 & 55637 & 136.9071 & 1 & 67 & 0 & 0 & 1 & 0 & 0 & 0 & Cs-137 \\
\hline 250 & 56634 & 133.9043 & 1 & 68 & 0 & 0 & 1 & 0 & 0 & 0 & Вa-134 \\
\hline 251 & 56637 & 136.9051 & 1 & 69 & 0 & 0 & 1 & 0 & 0 & 0 & Ba-137 \\
\hline 252 & 56640 & 139.9109 & 1 & 70 & 0 & 0 & 1 & 0 & 0 & 0 & Ba-140 \\
\hline 253 & 57639 & 138.9063 & 1 & 71 & 0 & 0 & 1 & 0 & 0 & 0 & La-139 \\
\hline 254 & 57640 & 139.9099 & 1 & 72 & 0 & 0 & 1 & 0 & 0 & 0 & La-140 \\
\hline 255 & 58640 & 139.9059 & 1 & 73 & 0 & 0 & 1 & 0 & 0 & 0 & Ce-140 \\
\hline 256 & 58641 & 140.9085 & 1 & 74 & 0 & 0 & 1 & 0 & 0 & 0 & Ce-141 \\
\hline 257 & 58642 & 141.9091 & 1 & 75 & 0 & 0 & 1 & 0 & 0 & 0 & Ce-142 \\
\hline 258 & 58643 & 142.9127 & 1 & 76 & 0 & 0 & 1 & 0 & 0 & 0 & Ce-143 \\
\hline 259 & 58644 & 143.9133 & 1 & 77 & 0 & 0 & 1 & 0 & 0 & 0 & Ce-144 \\
\hline 260 & 59641 & 140.9075 & 1 & 78 & 0 & 3 & 1 & 0 & 0 & 0 & Pr-141 \\
\hline 261 & 59643 & 142.9107 & 1 & 79 & 0 & 0 & 1 & 0 & 0 & 0 & Pr-143 \\
\hline 262 & 60642 & 141.9081 & 1 & 80 & 0 & 0 & 1 & 0 & 0 & 0 & Nd-142 \\
\hline 263 & 60644 & 143.9103 & 1 & 82 & 0 & 0 & 1 & 0 & 0 & 0 & Nd-144 \\
\hline 264 & 60646 & 145.9135 & 1 & 84 & 0 & 3 & 1 & 0 & 0 & 0 & Nd-146 \\
\hline 265 & 60647 & 146.9161 & 1 & 85 & 0 & 0 & 1 & 0 & 0 & 0 & Nd-147 \\
\hline 266 & 60648 & 147.9167 & 1 & 86 & 0 & 3 & 1 & 0 & 0 & 0 & Nd-148 \\
\hline 267 & 60650 & 149.9209 & 1 & 87 & 0 & 3 & 1 & 0 & 0 & 0 & Nd-150 \\
\hline 268 & 61648 & 147.9177 & 1 & 89 & 0 & 0 & 1 & 0 & 0 & 0 & Pm-148 \\
\hline 269 & 61649 & 148.9183 & 1 & 90 & 0 & 0 & 1 & 0 & 0 & 0 & Pm-149 \\
\hline 270 & 61651 & 150.9215 & 1 & 91 & 0 & 0 & 1 & 0 & 0 & 0 & Pm-151 \\
\hline 271 & 62648 & 147.9147 & 1 & 94 & 0 & 0 & 1 & 0 & 0 & 0 & Sm-148 \\
\hline 272 & 62653 & 152.9217 & 1 & 99 & 0 & 0 & 1 & 0 & 0 & 0 & Sm-153 \\
\hline 273 & 62654 & 153.9223 & 1 & 100 & 0 & 0 & 1 & 0 & 0 & 0 & Sm-154 \\
\hline 274 & 63651 & 150.9195 & 1 & 101 & 0 & 3 & 1 & 0 & 0 & 0 & Eu-151 \\
\hline
\end{tabular}




\begin{tabular}{|l|l|l|l|l|l|l|l|l|l|l|c|}
\hline 275 & 63656 & 155.9245 & 1 & 105 & 0 & 0 & 1 & 0 & 0 & 0 & Eu-156 \\
\hline 276 & 63657 & 156.9251 & 1 & 106 & 0 & 0 & 1 & 0 & 0 & 0 & Eu-157 \\
\hline 277 & 64654 & 153.9213 & 1 & 107 & 0 & 1 & 1 & 0 & 0 & 0 & Gd-154 \\
\hline 278 & 64660 & 159.9268 & 1 & 112 & 0 & 1 & 1 & 0 & 0 & 0 & Gd-160 \\
\hline 279 & 65159 & 158.9252 & 0 & 0 & 0 & 0 & 1 & 0 & 0 & 0 & Tb-159 \\
\hline 280 & 65160 & 158.9273 & 1 & 0 & 0 & 0 & 1 & 0 & 0 & 0 & Tb-160 \\
\hline 281 & 65161 & 160.9276 & 1 & 0 & 0 & 0 & 1 & 0 & 0 & 0 & Tb-161 \\
\hline 282 & 65659 & 158.9252 & 1 & 113 & 0 & 0 & 1 & 0 & 0 & 0 & Tb-159 \\
\hline 283 & 65660 & 158.9273 & 1 & 114 & 0 & 0 & 1 & 0 & 0 & 0 & Tb-160 \\
\hline 284 & 65661 & 160.9276 & 1 & 115 & 0 & 0 & 1 & 0 & 0 & 0 & Tb-161 \\
\hline 285 & 66660 & 159.9248 & 1 & 116 & 0 & 0 & 1 & 0 & 0 & 0 & Dy-160 \\
\hline 286 & 66661 & 160.9274 & 1 & 117 & 0 & 0 & 1 & 0 & 0 & 0 & Dy-161 \\
\hline 287 & 66662 & 161.9270 & 1 & 118 & 0 & 0 & 1 & 0 & 0 & 0 & Dy-162 \\
\hline 288 & 66663 & 162.9286 & 1 & 119 & 0 & 0 & 1 & 0 & 0 & 0 & Dy-163 \\
\hline 289 & 66664 & 163.9282 & 1 & 120 & 0 & 3 & 1 & 0 & 0 & 0 & Dy-164 \\
\hline 290 & 67165 & 164.9298 & 0 & 0 & 0 & 3 & 1 & 0 & 0 & 0 & Ho-165 \\
\hline 291 & 67665 & 164.9298 & 1 & 121 & 0 & 3 & 1 & 0 & 0 & 0 & Ho-165 \\
\hline 292 & 77191 & 190.9781 & 0 & 0 & 0 & 0 & 1 & 0 & 0 & 0 & Ir-191 \\
\hline 293 & 77193 & 192.9803 & 0 & 0 & 0 & 0 & 1 & 0 & 0 & 0 & Ir-193 \\
\hline 294 & 91232 & 232.0386 & 1 & 0 & 0 & 0 & 1 & 0 & 0 & 0 & Pa-232 \\
\hline 295 & 91234 & 234.0433 & 1 & 0 & 0 & 0 & 1 & 0 & 0 & 0 & Pa-234 \\
\hline
\end{tabular}




\section{Appendix C.2 DECLIB input to generate the ENDF/B-7.1 V4.0 MPACT 47-group library}

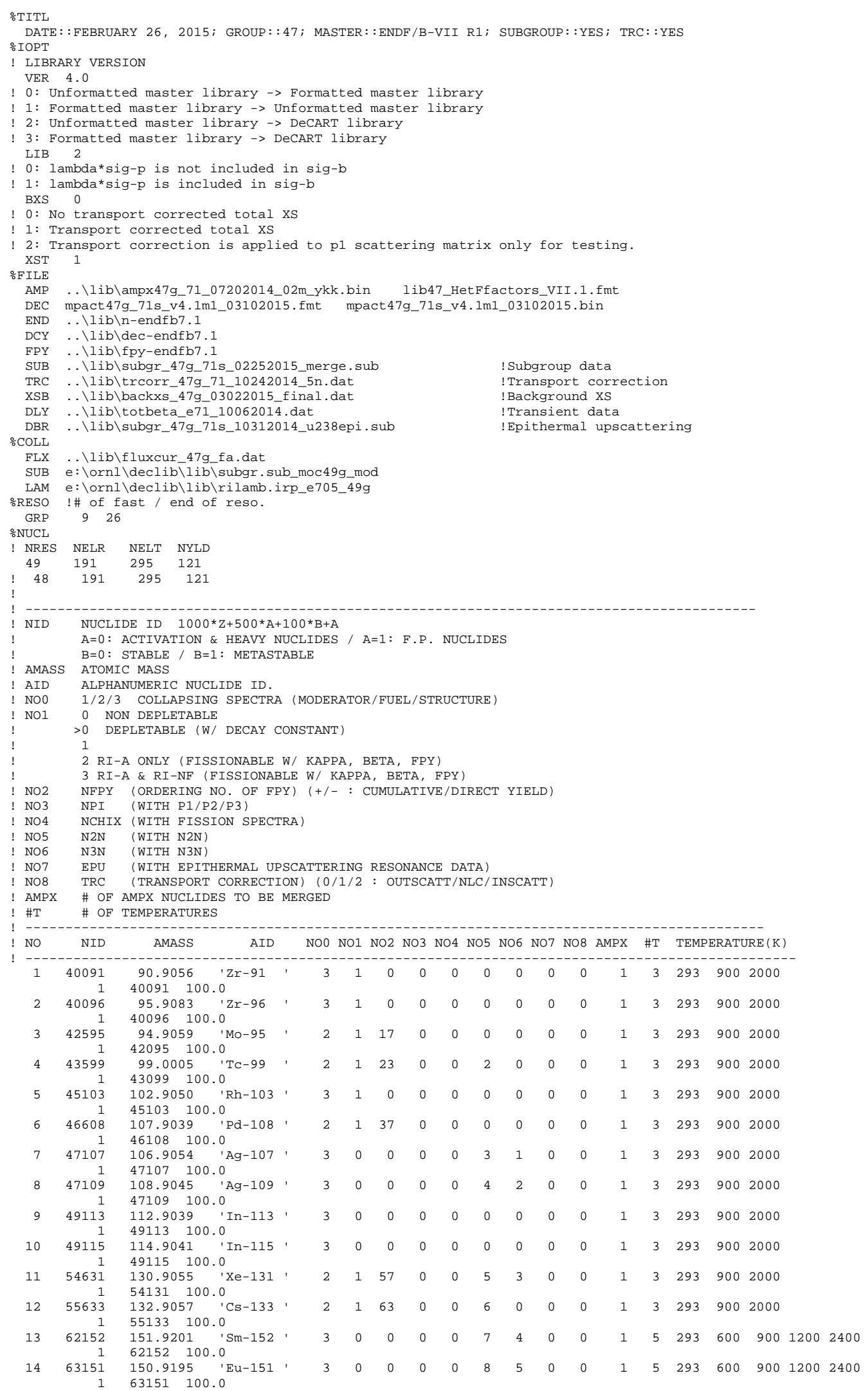




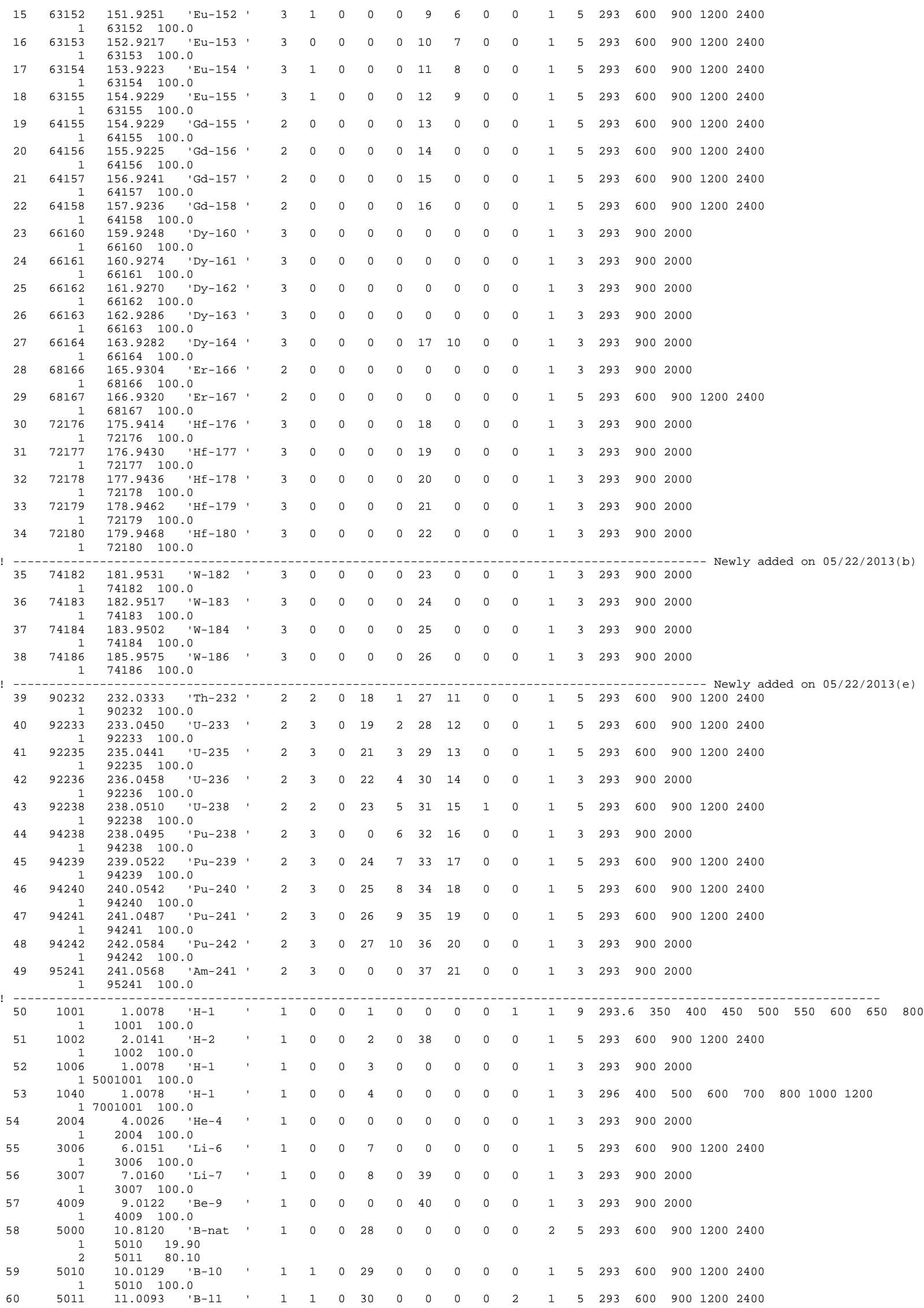




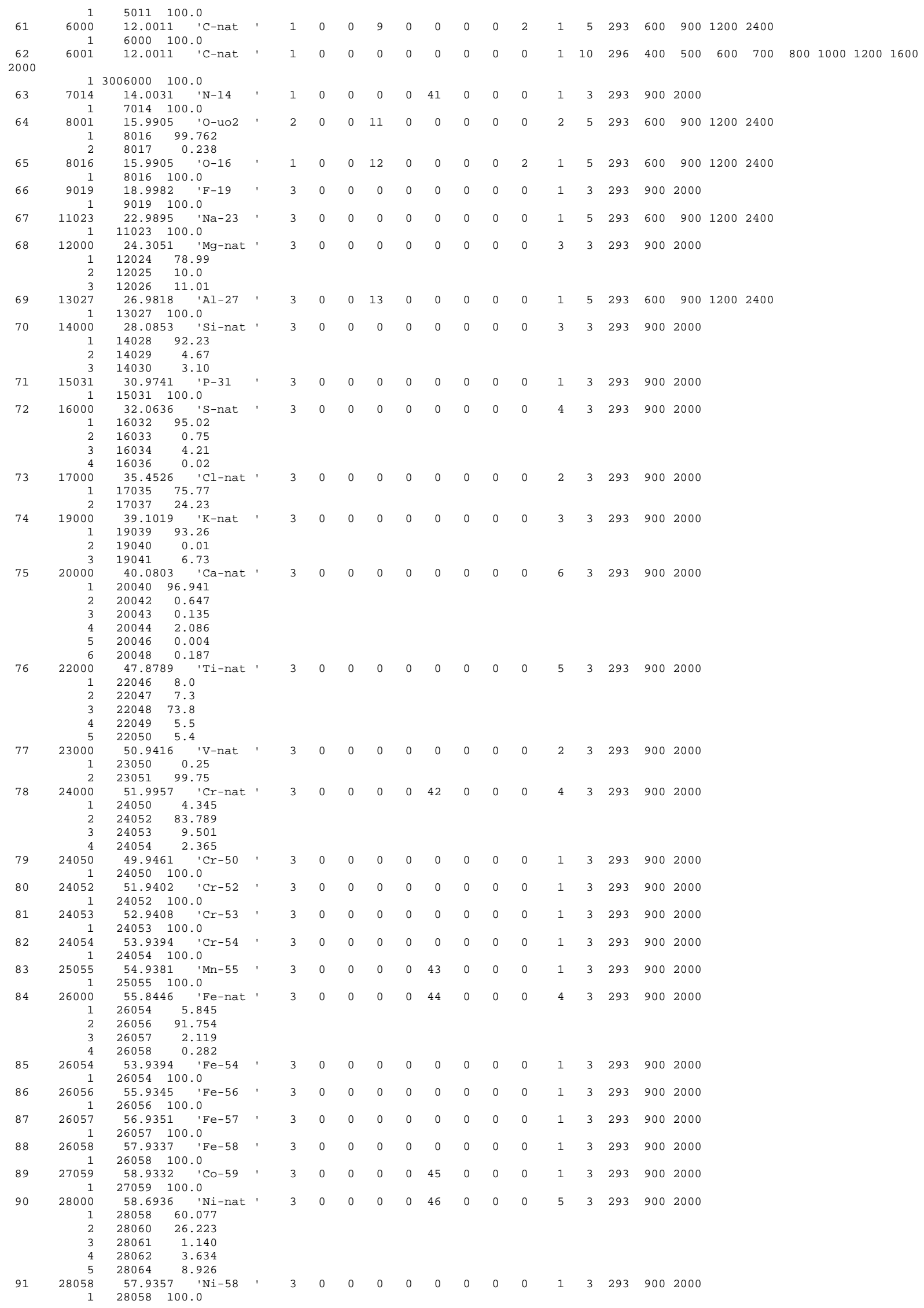



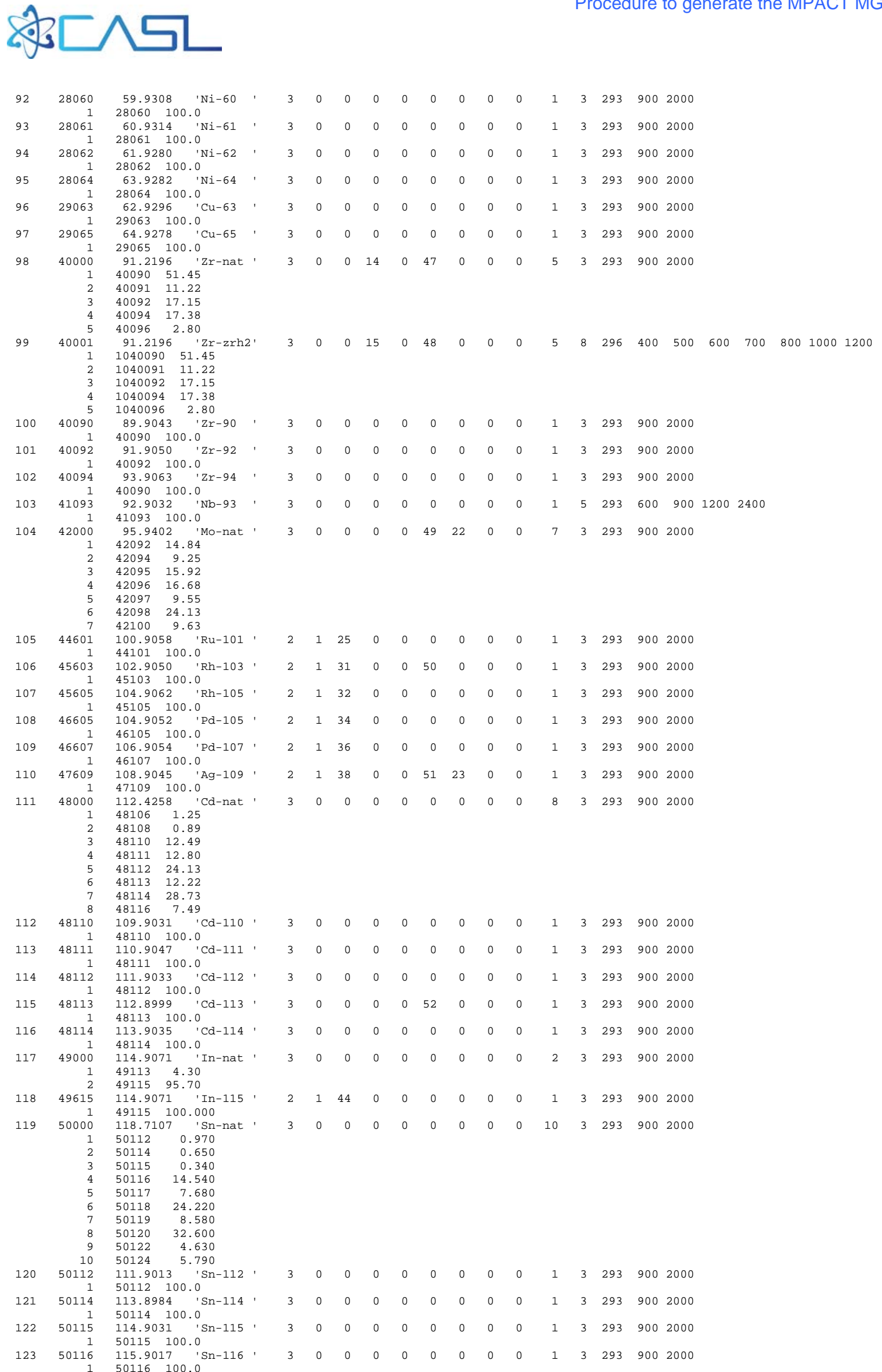


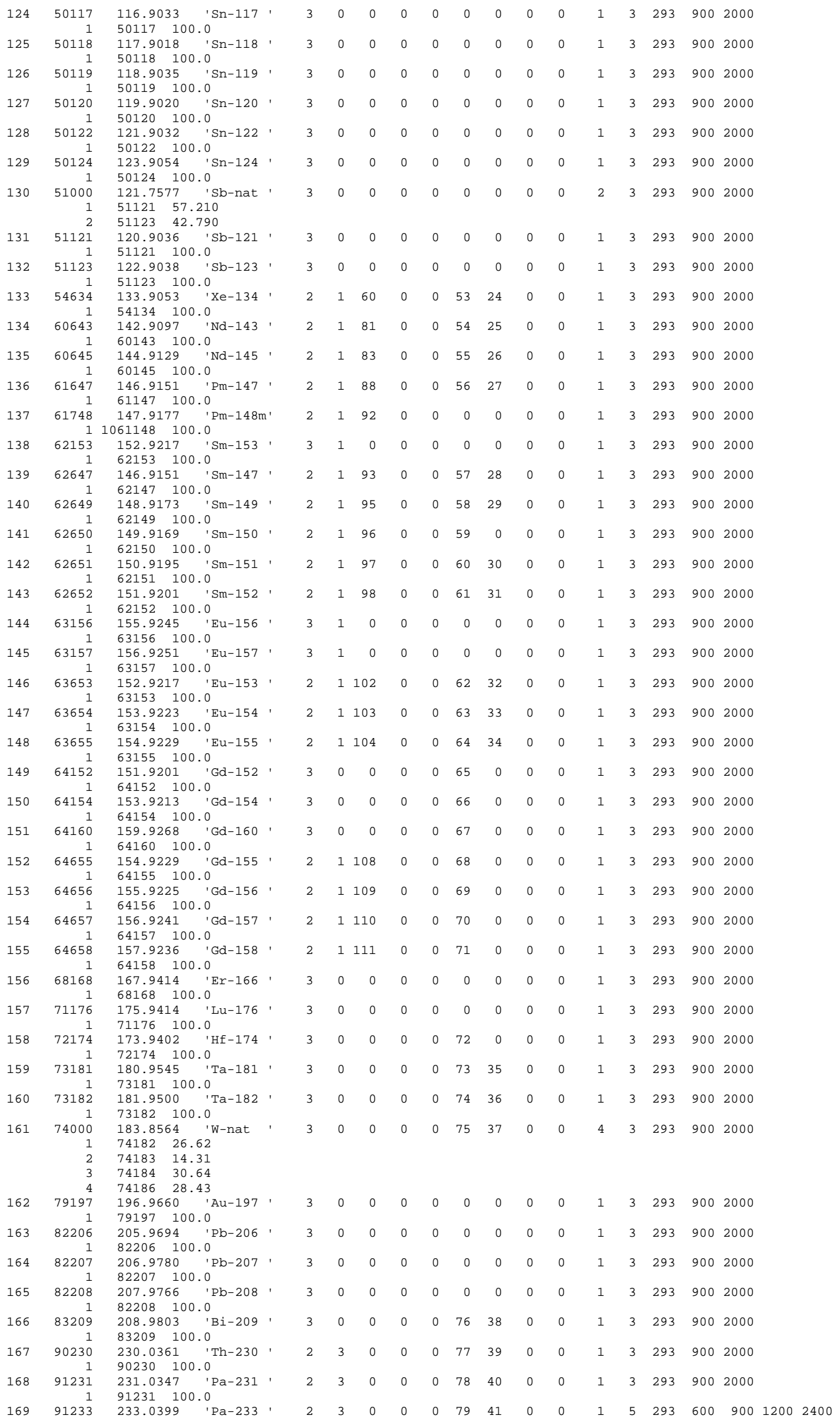




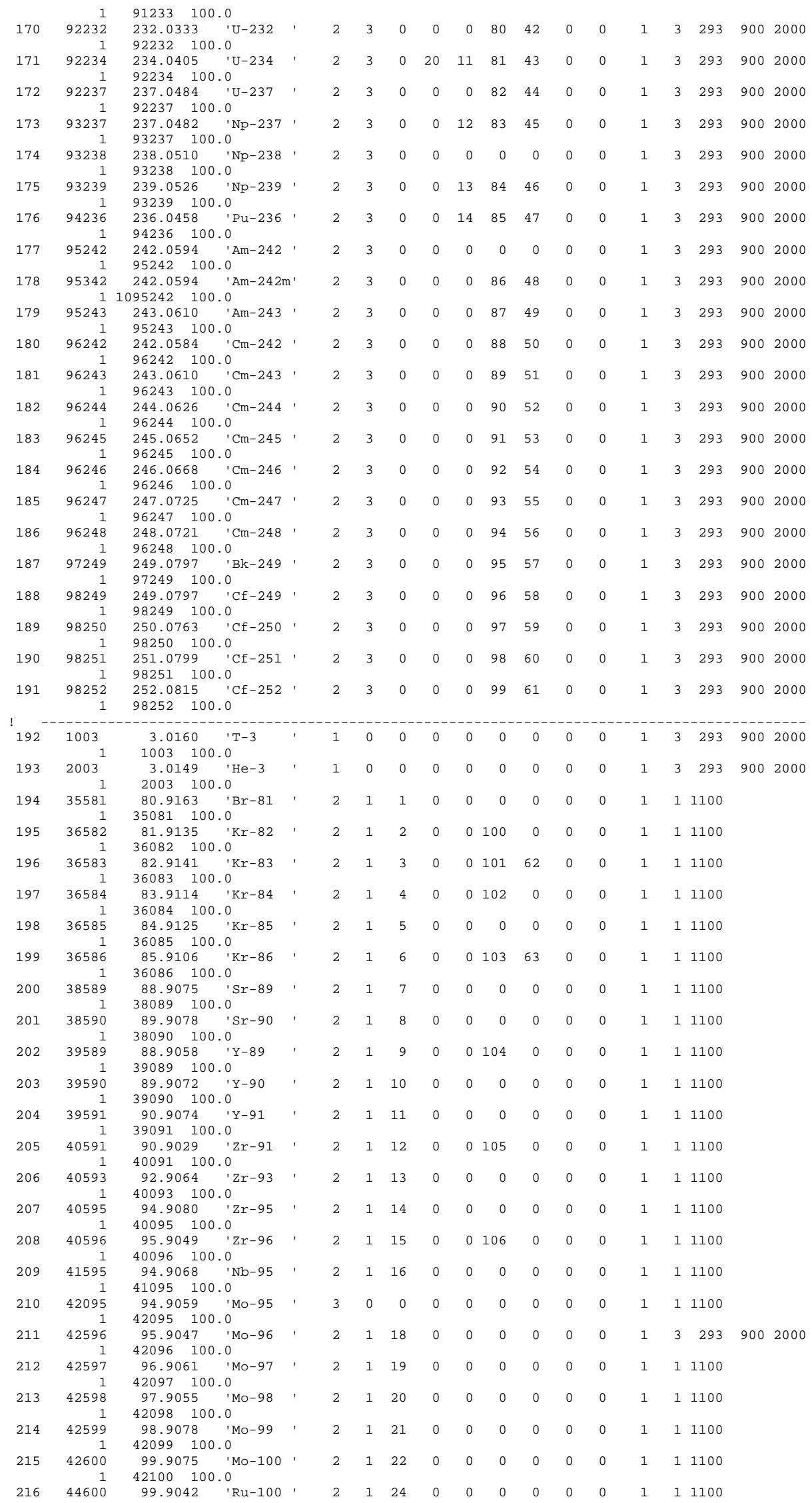




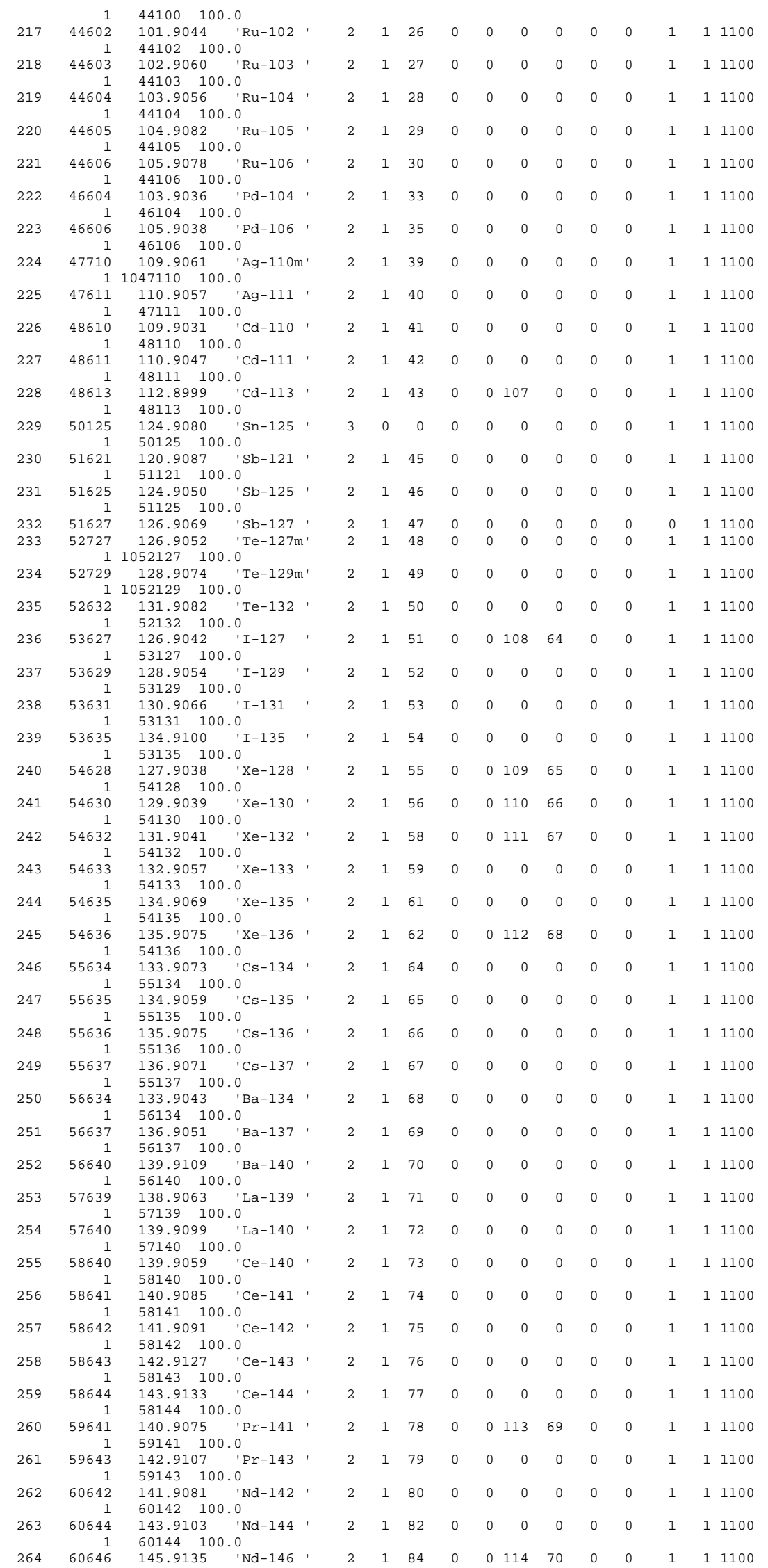




\section{玉닫ㄴ}

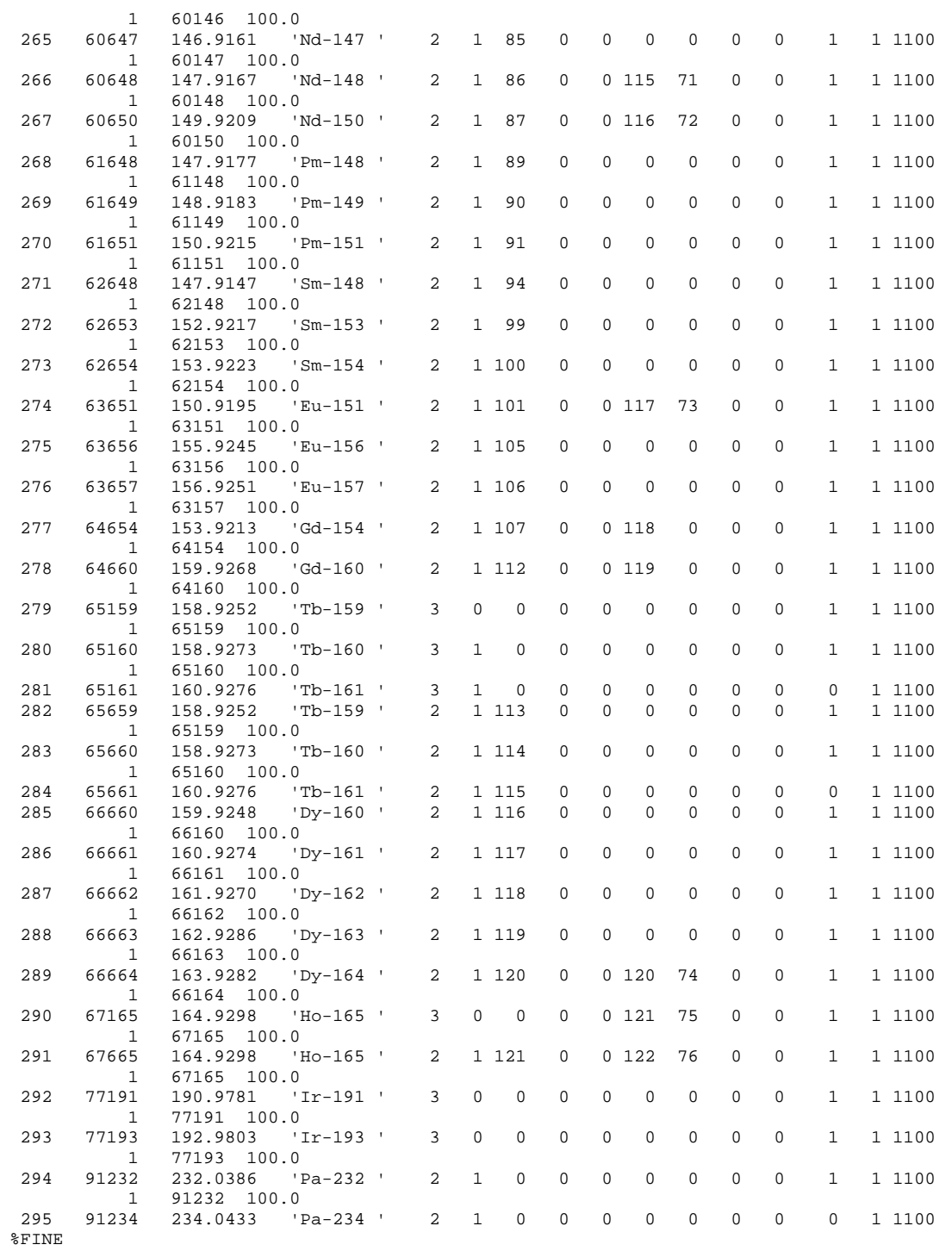




\section{Appendix C.3 Structure of the V4.0 MPACT MG library}

\section{[Structure]}

\%VERSION:

[iversion, adte]

\%DIM:

[nog, nofg, norg, nchi, nelt, nelr, nres, nyld, nsubg, nflxhel]

\%GRP:

[enbhel(ig) $]_{\text {ig }=1, \text { nog }}$

\%CHI:

$[\text { chix(ig) }]_{\mathrm{ig}=1, \mathrm{nog}}$

\%DIR:

do $\mathrm{i}=1$, nelt

[ix, nid, amass, ityp, ifpy, ichi, inmn, ntemp, nrtemp, np1temp, npot, aid] enddo

\%NUC: [i, nid, amass, ityp, ifpy, ichi, inmn, ntemp, nrtemp, np1temp, npot, aid] ["TP1+"]

[temp(it)] $]_{\mathrm{it}=1, \text { ntemp }}$

["XSD+"]

do ig=1,nog

do it=1,ntemp

[ig, it, siga, \{sigf, signf, sigtr, sigs, ib,ie, sm(jg) $)_{\mathrm{jg}=\mathrm{ib}}$, ie $\}$ ]

enddo

enddo

[" SP1+"]

do ig=1,nog

do it=1,np1temp

[ig, it, sigsp1(ig,it), ib, ie, smp1(jg,it $)_{\mathrm{jg}=\mathrm{ib}, \mathrm{ie}}$ ] enddo

enddo

["POT+"]

$\left\{[\text { lamsigp(ig) }]_{\text {ig=igresb,igrese }}\right.$

[sigsres(ig)] ig=igresb,igrese

$[\mathrm{nu}(\mathrm{ig})]_{\text {ig=igresb,igrese }}$

[" TP2+"]

[rtemp(it)] $]_{\mathrm{it}=1, \mathrm{nrtemp}}$

[" XSO+"]

[sigpot(ip)] ip=1,npot

[" RIA+"]

do ig=igresb,igrese

do it=1,ldiso(i)\%nrtemp

[ig, it, ria(ip,it,ig) $\left.)_{\mathrm{ip}=1, \text { npot }}\right]_{3}$ enddo

enddo

["RIS+"]

do ig=igresb,igrese

do it=1,ldiso(i)\%nrtemp

[ig, it, ris(ip,it,ig) $\left.{ }_{\mathrm{ip}=1, \mathrm{npot}}\right]$ enddo 


\section{※단}

enddo

["RNF+"]

do ig=igresb,igrese

do it=1,ldiso(i)\%nrtemp

[ig, it, rinf(ip,it,ig) $)_{\mathrm{ip}=1, \text { npot }}$ ]

enddo

enddo

["SA7+"]

do ig=igresb,igrese

[ig, sgsa(ix,ig) ${ }_{\mathrm{ix}=1, \mathrm{nsubg}}$ ]

enddo

do ig=igresb,igrese

do it=1,ldiso(i)\%nrtemp

[ig, it, sgwa(ix,it,ig) ${ }_{\mathrm{ix}=1, \text { nsubg }}$ ] enddo

enddo

["SF7+"]

do ig=igresb,igrese

[ig, $\left.\operatorname{sgsnf}(i x, i g)_{i x=1, n s u b g}\right]$

enddo

do ig=igresb,igrese

do it=1,ldiso(i)\%nrtemp

[ig, it, sgwnf(ix,it,ig) $)_{\mathrm{ix}=1, \text { nsubg }}$ ] enddo

enddo

["SA4+"]

do ig=igresb,igrese

[ig, sgsafsp(ix,ig) $)_{\mathrm{ix}=1, \mathrm{nflx}}$ ]

enddo

do ig=igresb,igrese +1

do it=1,ldiso(i)\%nrtemp

[ig, it, sgwafsp(ix,it,ig) $\left.)_{\mathrm{ix}=1, \mathrm{nflx}}\right]$

enddo

enddo

["CHI+"]

[chi(ig)] $]_{\text {ig=1,nog }}$

["CHI-"]

[chid(ig)] $]_{\mathrm{ig}=1, \mathrm{nog}}$

[" FIS+"]

[kappa, kappa0]

[beta(ib)] ib=0,6

[dcybeta(ib)] $\mathrm{ib}=1,6$

[yield(iy)] iy=1,nyld

[" DCY+"]

[ldiso(i)\%dcy]

[" TP3+"]

[ldiso(i)\%p1temp(it)] $]_{\text {it=1,np1temp }}$

[" SP2+"]

do ig $=1$, nog

do it=1,np1temp

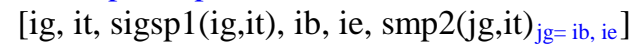
enddo

enddo

[" SP3+"]

do ig $=1, \operatorname{nog}$

do it=1,np1temp 



enddo

enddo

[" N2N+"]

[ldiso(i)\%sign2n(ig) $]_{\text {ig=1,nog }}$

[" N3N+"]

[ldiso(i)\%sign3n(ig) $]_{\text {ig=1,nog }}$

$* *$ Repeat \%NUC: for nelt nuclides.

\%END:

\begin{tabular}{|c|c|c|}
\hline Variables & Type & Description \\
\hline nog & I & The number of energy groups \\
\hline nofg & $\mathrm{I}$ & The number of fast energy groups \\
\hline norg & $\mathrm{I}$ & The number of resonance energy groups \\
\hline nchi & $\mathrm{I}$ & The number of energy groups w/ fission spectra \\
\hline nelt & I & The total number of nuclides \\
\hline nelr & I & The number of nuclides $\mathrm{w} /$ full xs sets \\
\hline nyld & $\mathrm{I}$ & The number of fission product nuclides \\
\hline nres & $\mathrm{I}$ & The number of resonant nuclides \\
\hline nsubg & $\mathrm{I}$ & The number of subgroup levels \\
\hline nflx & $\mathrm{I}$ & The number of coarse subgroup levels \\
\hline igresb & I & Starting group for resonance $(=$ nofg+1) \\
\hline igrese & $\mathrm{I}$ & Ending group for resonance (=nofg+norg) \\
\hline u(ig) & $\mathrm{R}$ & Lethargy energy group boundaries (ig=1,nog) \\
\hline enb(ig) & $\mathrm{R}$ & Energy group boundaries (ig=1,nog) \\
\hline chix(ig) & $\mathrm{R}$ & Common fission spectrum (ig=1,nog) \\
\hline $\operatorname{sm}(p ?)(i, j) \%$ & $\mathrm{~T}$ & [scatmat] P0 p3 scattering matrix \\
\hline$\%$ ib & $\mathrm{I}$ & Stating energy group (in) \\
\hline \%ie & $\mathrm{I}$ & Ending energy group (in) \\
\hline \%ioutsb & $\mathrm{I}$ & Stating energy group (out) \\
\hline \%ioutse & $\mathrm{I}$ & Ending energy group (out) \\
\hline \%from(k) & $\mathrm{R}$ & Scattering matrix (k=\%ib,\%ie) \\
\hline ldiso(i)\% & $\mathrm{T}$ & [libdata] Nuclidewise multigroup data $\quad(\mathrm{i}=1$, nelt) \\
\hline \%nid & $\mathrm{I}$ & Nuclide id. \\
\hline \multirow{5}{*}{ \%ityp } & \multirow{5}{*}{$\mathrm{I}$} & Indicator for data type \\
\hline & & 0 non depletable \\
\hline & & $>0$ depletable (w/ decay constant) \\
\hline & & 2 ri-a (fissionable w/ kappa, beta, fpy) \\
\hline & & 3 ri-a \& ri-nf (same as above) \\
\hline \%ichi & $\mathrm{I}$ & Indicator for fission spectrum $(0 />0:$ no/yes $)$ \\
\hline \%ifpy & $\mathrm{I}$ & Indicator \& ordering no. for f.p.y. \\
\hline \%inmn & $\mathrm{I}$ & Indicator for $(n, 2 n) \&(n, 3 n)$ xs $(0 / 1 / 2 / 3: n o /(n, 2 n) /(n, 3 n) / b o t h)$ \\
\hline \%ntemp & I & The number of temperatures for xs \\
\hline \%nrtemp & $\mathrm{I}$ & The number of temperatures for ri \\
\hline \%npot & $\mathrm{I}$ & The number of sig-0's for ri \\
\hline \%np1temp & $\mathrm{I}$ & The number of temperatures for p1 p3 \\
\hline \%aid & A8 & Alphanumeric nuclide id. \\
\hline \%aw & $\mathrm{R}$ & Atomic mass \\
\hline \%kappa & $\mathrm{R}$ & Energy release per fission (w/sec) only w/o neutrino energy \\
\hline \%kappa0 & $\mathrm{R}$ & Energy release per fission (w/sec) w/o decay and neutron kinetic energy \\
\hline \%crit_nd & $\mathrm{R}$ & Critical pnd \\
\hline \%dcy & $\mathrm{R}$ & Decay constant (/sec) \\
\hline
\end{tabular}




\begin{tabular}{|c|c|c|}
\hline \%chi(j) & $\mathrm{R}$ & Fission spectrum $(\mathrm{j}=1, \mathrm{nog})$ \\
\hline \%chid(j) & $\mathrm{R}$ & Delayed neutron spectrum ( $\mathrm{j}=1, \mathrm{nog})$ \\
\hline \%beta(j) & $\mathrm{R}$ & Delayed neutron yields $(j=1,6)$ \\
\hline \%dcybeta(j) & $\mathrm{R}$ & Delayed neutron decay constant $(\mathrm{j}=1,6)$ \\
\hline \%rtemp(j) & $\mathrm{R}$ & Square root temperatures for ri ( $\mathrm{j}=1, \%$ nrtemp) \\
\hline \%sigpot(j) & $\mathrm{R}$ & Square root sig-0's for ri ( $\mathrm{j}=1, \%$ npot) \\
\hline \%temp(j) & $\mathrm{R}$ & Temperatures for xs ( $\mathrm{j}=1, \%$ ntemp) \\
\hline \%p1temp(j) & $\mathrm{R}$ & Temperatures for p1 p3 (j=1,\%np1temp) \\
\hline \%lamsigp(j) & $\mathrm{R}$ & Lambda*potential xs ( $\mathrm{j}=$ igresb,igrese) \\
\hline \%sigsres(j) & $\mathrm{R}$ & Scattering xs (j=igresb,igrese) \\
\hline$\%$ nu(j) & $\mathrm{R}$ & The number of neutrons released (j=igresb,igrese) \\
\hline \%yield(j,k) & $\mathrm{R}$ & Fission product yields $(\mathrm{j}=1, ; \mathrm{k}=1, \mathrm{)}$ \\
\hline \%siga(j,k) & $\mathrm{R}$ & Absorption xs (j=1,nog; $k=1, \%$ ntemp) \\
\hline \%sigf(j,k) & $\mathrm{R}$ & Fission xs ( $\mathrm{j}=1, \mathrm{nog} ; \mathrm{k}=1, \%$ ntemp) \\
\hline \%signf(j,k) & $\mathrm{R}$ & Nu*fission xs (j=1,nog; k=1,\%ntemp) \\
\hline \%sigtr(j,k) & $\mathrm{R}$ & Transport xs (j=1,nog; $k=1, \%$ ntemp) \\
\hline \%sigs(j,k) & $\mathrm{R}$ & Scattering xs ( $\mathrm{j}=1$, nog; $\mathrm{k}=1, \%$ ntemp) \\
\hline \%sigstr(j,k) & $\mathrm{R}$ & Transport correct scattering xs ( $\mathrm{j}=1, \mathrm{nog} ; \mathrm{k}=1, \%$ ntemp) \\
\hline \%sigsp1(j,k) & $\mathrm{R}$ & P1 scattering xs ( $\mathrm{j}=1$, nog; $\mathrm{k}=1, \%$ np1temp) \\
\hline \%sigsp2(j,k) & $\mathrm{R}$ & P2 scattering xs ( $\mathrm{j}=1$, nog; $\mathrm{k}=1, \%$ np1temp) \\
\hline \%sigsp3(j,k) & $\mathrm{R}$ & P3 scattering xs ( $\mathrm{j}=1$, nog; $\mathrm{k}=1, \%$ np1temp) \\
\hline \%sign2n(j) & $\mathrm{R}$ & $(n, 2 n) x s(j=1, n o g)$ \\
\hline \%sign3n(j) & $\mathrm{R}$ & $(n, 3 n)$ xs $(j=1, n o g)$ \\
\hline$\% \operatorname{sm}(\mathrm{j}, \mathrm{k}) \%$ & $\mathrm{~T}$ & [scatmat] P0 corrected scattering matrix ( $\mathrm{j}=1, \mathrm{nog} ; \mathrm{k}=1, \%$ np1temp) \\
\hline \%smp1(j,k)\% & $\mathrm{T}$ & [scatmat] P1 scattering matrix ( $\mathrm{j}=1, \mathrm{nog} ; \mathrm{k}=1, \% \mathrm{np} 1$ temp) \\
\hline \%smp2(j,k)\% & $\mathrm{T}$ & [scatmat] P2 scattering matrix ( $\mathrm{j}=1, \mathrm{nog} ; \mathrm{k}=1, \% \mathrm{np} 1$ temp) \\
\hline \%smp3(j,k)\% & $\mathrm{T}$ & [scatmat] P3 scattering matrix ( $\mathrm{j}=1, \mathrm{nog} ; \mathrm{k}=1, \% \mathrm{np} 1$ temp) \\
\hline \%ria(j,k,l) & $\mathrm{R}$ & Absorption ri (j=1,\%npot; k=1,\%nrtemp; l=igresb,igrese) \\
\hline \%rinf(j,k,l) & $\mathrm{R}$ & Nu*fission ri (j=1,\%npot; k=1,\%nrtemp; l=igresb,igrese) \\
\hline \%ris(j,k,l) & $\mathrm{R}$ & Scattering ri ( $\mathrm{j}=1, \%$ npot; $\mathrm{k}=1, \%$ nrtemp; l=igresb,igrese) \\
\hline \%sgsa(j,k) & $\mathrm{R}$ & Subgroup levels of absorption ri ( $\mathrm{j}=1$,nsubg; $\mathrm{k}=$ igresb,igrese) \\
\hline \%sgsnf(j,k) & $\mathrm{R}$ & Subgroup levels of nu*fission ri ( $\mathrm{j}=1$,nsubg; $\mathrm{k}=$ igresb,igrese) \\
\hline \%sgsafsp(j,k) & $\mathrm{R}$ & Subgroup levels of absorption ri $(\mathrm{j}=1, \mathrm{nfl}$; $\mathrm{k}=$ igresb,igrese +1$)$ \\
\hline \%sgwa(j,k,l) & $\mathrm{R}$ & $\begin{array}{l}\text { Subgroup weights of absorption ri } \\
\text { (j=1,nsubg; } k=1, \% \text { nrtemp; } k=\text { igresb,igrese) }\end{array}$ \\
\hline \%sgwnf(j,k,l) & $\mathrm{R}$ & $\begin{array}{l}\text { Subgroup weights of nu*fission ri } \\
\text { (j=1,nsubg; } k=1, \% \text { nrtemp; } k=\text { igresb,igrese) }\end{array}$ \\
\hline \%sgwafsp(j,k,l) & $\mathrm{R}$ & $\begin{array}{l}\text { Subgroup weights of absorption ri } \\
(\mathrm{j}=1, \text { nflx; } k=1, \% \text { nrtemp; } k=\text { igresb,igrese }+1)\end{array}$ \\
\hline
\end{tabular}

